\title{
WELL-POSEDNESS FOR THE FIFTH-ORDER KDV EQUATION IN THE ENERGY SPACE
}

\author{
CARLOS E. KENIG AND DIDIER PILOD
}

\begin{abstract}
We prove that the initial value problem (IVP) associated to the fifth-order $\mathrm{KdV}$ equation

$$
\partial_{t} u-\partial_{x}^{5} u=c_{1} \partial_{x} u \partial_{x}^{2} u+c_{2} \partial_{x}\left(u \partial_{x}^{2} u\right)+c_{3} \partial_{x}\left(u^{3}\right),
$$

where $x \in \mathbb{R}, t \in \mathbb{R}, u=u(x, t)$ is a real-valued function and $\alpha, c_{1}, c_{2}, c_{3}$ are real constants with $\alpha \neq 0$, is locally well-posed in $H^{s}(\mathbb{R})$ for $s \geq 2$. In the Hamiltonian case $\left(i . e\right.$. when $c_{1}=c_{2}$ ), the IVP associated to (0.1) is then globally well-posed in the energy space $H^{2}(\mathbb{R})$.
\end{abstract}

\section{INTRODUCTION}

Considered here is the initial value problem (IVP) associated to the fifth-order Korteweg-de Vries equation

$$
\left\{\begin{array}{l}
\partial_{t} u-\partial_{x}^{5} u=c_{1} \partial_{x} u \partial_{x}^{2} u+c_{2} \partial_{x}\left(u \partial_{x}^{2} u\right)+c_{3} \partial_{x}\left(u^{3}\right) \\
u(\cdot, 0)=u_{0}
\end{array}\right.
$$

where $x \in \mathbb{R}, t \in \mathbb{R}, u=u(x, t)$ is a real-valued function and $c_{1}, c_{2}, c_{3}$ are real constants. Such equations and their generalizations

$$
\partial_{t} u-\partial_{x}^{5} u+\beta \partial_{x}^{3} u=c_{0} u \partial_{x} u+c_{1} \partial_{x} u \partial_{x}^{2} u+c_{2} \partial_{x}\left(u \partial_{x}^{2} u\right)+c_{3} \partial_{x}\left(u^{3}\right)
$$

arise as long-wave approximations to the water-wave equation. They have been derived as second-order asymptotic expansions for unidirectional wave propagation in the so-called Boussinesq regime (see Craig, Guyenne and Kalisch [5, Olver 31, Craig and Groves [6] and the references therein), the first-order expansions being of course the Korteweg-de Vries (KdV) equation,

$$
\partial_{t} u+\beta \partial_{x}^{3} u=c_{0} u \partial_{x} u .
$$

The equation in (1.1) was also proposed by Benney [2] as a model for interaction of short and long waves.

When $c_{1}=c_{2}$, the Hamiltonian

$$
H(u)=\frac{1}{2} \int_{\mathbb{R}}\left(\left(\partial_{x}^{2} u\right)^{2}-c_{1} u\left(\partial_{x} u\right)^{2}+\frac{c_{3}}{2} u^{4}\right) d x
$$

Received by the editors May 3, 2012 and, in revised form, June 23, 2012 and October 6, 2012. 2010 Mathematics Subject Classification. Primary 35Q53, 35Q35, 35A01; Secondary 37K05, $76 \mathrm{~B} 15$.

Key words and phrases. Fifth-order KdV equation, fifth-order water-waves models, initial value problem.

The first author was partially supported by NSF Grant DMS-0968472.

The second author was partially supported by CNPq/Brazil, Grant 200001/2011-6. 
as well as the quantity

$$
M(u)=\int_{\mathbb{R}} u^{2} d x,
$$

are conserved by the flow of (1.1). Indeed, it is easy to check that

$$
H^{\prime}(u) \varphi=\int_{\mathbb{R}}\left(\partial_{x}^{4} u-\frac{c_{1}}{2}\left(\partial_{x} u\right)^{2}+c_{1} \partial_{x}\left(u \partial_{x} u\right)+c_{3} u^{3}\right) \varphi d x=:(\operatorname{grad} H(u), \varphi)_{L^{2}} .
$$

Thus the equation in (1.1) has the form $\partial_{t} u=\partial_{x} \operatorname{grad} H(u)$, so that

$$
\frac{d}{d t} H(u)=\left(\operatorname{grad} H(u), \partial_{t} u\right)_{L^{2}}=\left(\operatorname{grad} H(u), \partial_{x} \operatorname{grad} H(u)\right)_{L^{2}}=0 .
$$

Moreover in the special case where $c_{2}=c_{1}=-10 \alpha$ and $c_{3}=10 \alpha$, the equation in (1.1) is the equation following $\mathrm{KdV}$ in the $\mathrm{KdV}$ hierarchy discovered by Lax 26] and written as

$$
\partial_{t} u-\partial_{x}^{5} u+10 \partial_{x} u \partial_{x}^{2} u+10 \partial_{x}\left(u \partial_{x}^{2} u\right)-10 \partial_{x}\left(u^{3}\right)=0 .
$$

Therefore equation (1.6) is completely integrable and possesses an infinite number of conservation laws. We refer to the introductions in [9, 33], 34] for more details on this subject.

Our purpose is to study the IVP (1.1) in classical $L^{2}$-based Sobolev spaces $H^{s}(\mathbb{R})$. We shall say that the IVP is locally (resp. globally) well-posed in the function space $X$ if it induces a dynamical system on $X$ by generating a continuous local (resp. global) flow.

First, it is worth mentioning that without dispersion (i.e. when $\alpha=0$ ) and when $c_{1} \neq 0$ or $c_{2} \neq 0$, the IVP (1.1) is likely to be ill-posed in any $H^{s}(\mathbb{R})$ (see the comments in the introduction of [33]). This is in sharp contrast with the KdV equation. Indeed, when $\beta=0$ in (1.3), we obtain the Burgers equation, which is still well-posed in $H^{s}(\mathbb{R})$ for $s>3 / 2$ by using standard energy methods. However, the direct energy estimate for equation (1.1) (after fixing $c_{3}=0$ for simplicity) gives only

$$
\frac{d}{d t}\left\|\partial_{x}^{k} u(t)\right\|_{L^{2}}^{2} \lesssim\left\|\partial_{x}^{3} u\right\|_{L_{x}^{\infty}}\left\|\partial_{x}^{k} u(t)\right\|_{L^{2}}^{2}+\left|\int_{\mathbb{R}} \partial_{x} u \partial_{x}^{k+1} u \partial_{x}^{k+1} u d x\right| .
$$

Observe that the last term on the right-hand side of (1.7) has still higher-order derivatives and cannot be treated by using only integration by parts. To overcome this difficulty, Ponce 33 ] used a recursive argument based on the dispersive smoothing effects associated to the linear part of (1.1), combined with a parabolic regularization method, to establish that the IVP (1.1) is locally well-posed in $H^{s}(\mathbb{R})$ for $s \geq 4$. Later, Kwon 25] improved Ponce's result by proving local well-posedness for (1.1) in $H^{s}(\mathbb{R})$ for $s>5 / 2$. The main new idea was to modify the energy by adding a correction lower-order cubic term to cancel the last term on the righthand side of (1.7). Note that he also used a refined Strichartz estimate derived by chopping the time interval into small pieces whose lengths depend on the spatial frequency. This estimate was first established by Koch and Tzvetkov [24] (see also Kenig and Koenig [18, for an improved version) in the Benjamin-Ono context.

On the other hand, it was proved 1 by the second author in 32, by using an argument due to Molinet, Saut and Tzvetkov for the Benjamin-Ono equation [30],

\footnotetext{
${ }^{1}$ Strictly speaking the result was proved only in the case where $c_{3}=0$, but as observed in the introduction of [9], the cubic term $\partial_{x}\left(u^{3}\right)$ in (1.1) is well behaved and no cancellations occur, so that the proof remains true even when $c_{3} \neq 0$.
} 
that in the case $c_{2} \neq 0$, the flow map associated to (1.1) fails to be $C^{2}$ in $H^{s}(\mathbb{R})$, for any $s \in \mathbb{R}$. This result was improved by Kwon 25, who showed that the flow map fails to be even uniformly continuous in $H^{s}(\mathbb{R})$ when $s>\frac{5}{2}$ (and $s>0$ in the completely integrable case). Those results are based on the fact that the dispersive smoothing effects associated to the linear part of (1.1) are not strong enough to control the high-low frequency interactions in the nonlinear term $\partial_{x}\left(u \partial_{x}^{2} u\right)$. As a consequence, one cannot solve the IVP (1.1) by a Picard iterative method implemented on the integral equation associated to (1.1) for initial data in any Sobolev space $H^{s}(\mathbb{R})$ with $s \in \mathbb{R}$.

However, the fixed point method may be employed to prove well-posedness for (1.1) in other function spaces. For example in [20, 21], Kenig, Ponce and Vega proved that the more general class of IVPs,

$$
\left\{\begin{array}{l}
\partial_{t} u+\partial_{x}^{2 j+1} u=P\left(u, \partial_{x} u, \ldots, \partial_{x}^{2 j} u\right), \quad x, t \in \mathbb{R}, j \in \mathbb{N} \\
u(0)=u_{0}
\end{array}\right.
$$

where

$$
P: \mathbb{R}^{2 j+1} \rightarrow \mathbb{R} \quad\left(\text { or } P: \mathbb{C}^{2 j+1} \rightarrow \mathbb{C}\right),
$$

is a polynomial having no constant or linear terms, is well-posed in weighted Sobolev spaces of the type $H^{k}(\mathbb{R}) \cap H^{l}\left(\mathbb{R} ; x^{2} d x\right)$ with $k, l \in \mathbb{Z}_{+}, k \geq k_{0}, l \geq l_{0}$ for some $k_{0}, l_{0} \in \mathbb{Z}_{+}$. We also refer to 32 for sharper results in the case of small initial data and when the nonlinearity in (1.8) is quadratic. Recently, Grünrock 9], respectively Kato [16], used variants of the Fourier restriction norm method to prove wellposedness in $\widehat{H}_{r}^{s}(\mathbb{R})$ for $1<r \leq \frac{4}{3}$ and $s>\frac{1}{4}+\frac{3}{2 r^{\prime}}$, respectively, in $H^{s, a}(\mathbb{R})$ for $s \geq \max \left\{-\frac{1}{4},-2 a-2\right\}$ with $-\frac{3}{2}<a \leq-\frac{1}{4}$ and $(s, a) \neq\left(-\frac{1}{4},-\frac{7}{8}\right)$. The spaces $\widehat{H}_{r}^{s}(\mathbb{R})$ and $H^{s, a}(\mathbb{R})$ are respectively defined by the norms $\|\varphi\|_{\widehat{H}_{r}^{s}}=\left\|\langle\xi\rangle^{s} \widehat{\varphi}\right\|_{L^{r^{\prime}}}$ with $\frac{1}{r}+\frac{1}{r^{\prime}}=1$ and $\|\varphi\|_{H^{s, a}}=\left\|\langle\xi\rangle^{s-a}|\xi|^{a} \widehat{\varphi}\right\|_{L^{2}}$.

Nevertheless, the $L^{2}$-based Sobolev spaces $H^{s}(\mathbb{R})$ remain the natura 2 spaces to study well-posedness for the fifth-order KdV equation. Our main result states that the IVP (1.1) is locally well-posed in $H^{s}(\mathbb{R})$ for $s \geq 2$.

Theorem 1.1. Assume that $s \geq 2$. Then, for every $u_{0} \in H^{s}(\mathbb{R})$, there exists a positive time $T=T\left(\left\|u_{0}\right\|_{H^{s}}\right)$ and a unique solution $u$ to (1.1) in the class

$$
C\left([-T, T] ; H^{s}(\mathbb{R})\right) \cap F^{s}(T) \cap B^{s}(T) .
$$

Moreover, for any $0<T^{\prime}<T$, there exists a neighborhood $\mathcal{U}$ of $u_{0}$ in $H^{s}(\mathbb{R})$ such that the flow map data-solution

$$
S_{T^{\prime}}^{s}: \mathcal{U} \longrightarrow C\left(\left[-T^{\prime}, T^{\prime}\right] ; H^{s}(\mathbb{R})\right), u_{0} \longmapsto u,
$$

is continuous.

Remark 1.2. The short-time Bourgain space $F^{s}(T):=F_{2}^{s}(T)$ and the energy space $B^{s}(T)$ are defined in Subsection 2.2 .

Remark 1.3. The result of Theorem 1.1 is also valid for equation (1.2) and the proof is similar.

\footnotetext{
${ }^{2}$ When the equation in (1.1) is Hamiltonian (i.e. when $c_{1}=c_{2}$ ), the space $H^{2}(\mathbb{R})$ is the natural space where the Hamiltonian $H$ in (1.4) is well defined.
} 
Remark 1.4. For sake of simplicity, we assume that $c_{3}=0$ in the proof of Theorem 1.1 since the cubic term $\partial_{x}\left(u^{3}\right)$ has low-order derivative when compared to the two other nonlinear terms in (1.1). Nevertheless, we indicate in the appendix what modifications are needed to deal with the case $c_{3} \neq 0$.

Remark 1.5. Observe that at this level of regularity $(s \geq 2)$, the limits of smooth solutions are still weak solutions to the equation in (1.1).

Remark 1.6. As a byproduct of the proof of Theorem 1.1 we obtain a priori estimates on smooth solutions of (1.1) in $H^{s}(\mathbb{R})$ for $s \geq \frac{5}{4}$ (see Proposition 6.2 below). In other words, the flow map data-solutions in $H^{\infty}(\mathbb{R})$ satisfy

$$
\left\|S_{T}^{\infty}\left(u_{0}\right)\right\|_{L_{T}^{\infty} H^{s}} \lesssim\left\|u_{0}\right\|_{H^{s}},
$$

for any $s \geq \frac{5}{4}$ and where $T$ depends only on $\left\|u_{0}\right\|_{H^{s}}$. However, we were not able to prove well-posedness at this level of regularity.

In the Hamiltonian case, the conserved quantities $H$ and $M$ defined in (1.4)(1.5) provide a control on the $H^{2}$-norm and allow us to prove that the IVP (1.1) is globally well-posed in $H^{2}(\mathbb{R})$.

Corollary 1.7. In the case $c_{1}=c_{2}$, the results of Theorem 1.1 are true for $T>0$ arbitrarily large.

Remark 1.8. Corollary 1.7 remains true for equation (1.2) (still in the case $c_{1}=c_{2}$ ).

Remark 1.9. In 34, Saut proved the existence of global weak solutions in the Hamiltonian case. However, Corollary 1.7 is the first existence result of global strong solutions for the fifth-order KdV equation in the Hamiltonian case 3

Remark 1.10. In his study of stability of solitary waves for Hamiltonian fifth-order water-wave models of the form (1.2) with quadratic nonlinearities 4 Levandosky assumed well-posedness in $H^{2}(\mathbb{R})$ (cf. Assumption 1.1 in [27]). Therefore, Corollary 1.7 provides an affirmative answer to this issue. We also refer to [1, 28] for further results on stability/instability of such fifth-order water-wave models.

We now discuss the main ingredients in the proof of Theorem 1.1. We follow the method introduced by Ionescu, Kenig and Tataru 15] in the context of the KP1 equation, which is based on the dyadic Bourgain spaces $F_{\alpha}^{s}$ and their dual $N_{\alpha}^{s}$, defined in subsection 2.2. We refer to [4], [23. for previous works using similar spaces to prove a priori bounds for the 1D cubic NLS at low regularity and also to [11, 12, 29] for applications to other dispersive equations.

The $F_{\alpha}^{s}$ spaces enjoy an $X^{s, b}$-type structure but with a localization in small time dependent intervals whose length is of order $2^{-\alpha k}$ when the spatial frequency of the function is localized around $2^{k}$. This prevents the time frequency modulation 5 $|\tau-w(\xi)|$ to be too small, which allows for suitable $\alpha, \alpha=2$ in our case, to prove a bilinear estimate of the form (cf. Proposition 4.1 for a precise statement)

$$
\left\|\partial_{x} u \partial_{x}^{2} v\right\|_{N_{2}^{s}(T)}+\left\|\partial_{x}\left(u \partial_{x}^{2} v\right)\right\|_{N_{2}^{s}(T)} \lesssim\|u\|_{F_{2}^{s}(T)}\|v\|_{F_{2}^{s}(T)}
$$

\footnotetext{
${ }^{3}$ Except of course in the completely integrable case.

${ }^{4}$ The question of existence of solitary waves for such models with nonhomogeneous nonlinearities was addressed in 22 .

${ }^{5}$ Here, $w(\xi)=\xi^{5}$ denotes the dispersive symbol of the linear equation.
} 
as soon as $s>1$. Of course 6 we cannot conclude directly by using a contraction argument since the linear estimate

$$
\|u\|_{F_{\alpha}^{s}(T)} \lesssim\|u\|_{B^{s}(T)}+\left\|\partial_{x} u \partial_{x}^{2} v\right\|_{N_{\alpha}^{s}(T)}+\left\|\partial_{x}\left(u \partial_{x}^{2} v\right)\right\|_{N_{\alpha}^{s}(T)}
$$

requires the introduction of the energy norm $\|u\|_{B^{s}(T)}$, instead of the usual $H^{s}$-norm of the initial data $\left\|u_{0}\right\|_{H^{s}}$, in order to control the small time localization appearing in the $F_{\alpha}^{s}$-structure. Therefore it remains to derive the frequency localized energy estimate

$$
\|u\|_{B^{s}(T)}^{2} \lesssim\left\|u_{0}\right\|_{H^{s}}^{2}+\left(1+\|u\|_{F^{s}(T)}\right)\|u\|_{F^{s}(T)}\left(\|u\|_{F^{s}(T)}^{2}+\|u\|_{B^{s}(T)}^{2}\right),
$$

which is true if $s \geq \frac{5}{4}$ and $\|u\|_{L_{T}^{\infty} H_{x}^{s}}$ is small. The main new difficulty in our case is that after using suitable frequency localized commutator estimates, we are not able to handle directly the remaining lower-order terms (see Lemma 5.7 and Remark 5.1 below). This is the price to pay for the choice of $\alpha=2$ which enabled us to derive the bilinear estimate (1.12). Then, we modify the energy by adding a cubic lower-order term to $\|u\|_{B^{s}(T)}^{2}$ in order to cancel those terms. This can be viewed as a localized version of Kwon's argument in 25].

We deduce the a priori bound (1.11) by combining (1.12)-(1.14) and using a scaling argument. To finish the proof of Theorem 1.1, we apply this method to the difference of two solutions. However, due to the lack of symmetry of the new equation, we only are able to prove the corresponding energy estimate for $s \geq 2$. Finally, we conclude the proof by adapting the classical Bona-Smith argument 3 .

We have learned that Guo, Kwak and Kwon [13 have also worked on the same problem and obtained the same results as ours (in Theorem 1.1 and Proposition 6.2). They also used the short-time $X^{s, b}$ method. However, instead of modifying the energy as we did, they put an additional weight in the $X^{s, b}$ structure of the spaces in order to derive the key energy estimates.

Finally, we believe that the technique employed here may be useful to deal with other higher-order nonlinear dispersive equations presenting the same kind of difficulties as the fifth-order KdV equation, as for example the other equations in the $\mathrm{KdV}$ hierarchy. We plan to address this issue in a forthcoming paper.

The rest of the paper is organized as follows: In Section 2, we introduce the notation, define the function spaces and prove some of their basic properties as well as the main linear estimates. In Section 3, we derive the $L^{2}$ bilinear and trilinear estimates, which are used to prove the bilinear estimates in Section 4 and the energy estimates in Section 5. The proof of Theorem 1.1 is given in Section 6. We conclude the paper with an appendix explaining how to treat the cubic term $\partial_{x}\left(u^{3}\right)$, which we omit in the previous sections to simplify the exposition.

\section{Notation, FUnCtion SPACES AND LineAR ESTimates}

2.1. Notation. For any positive numbers $a$ and $b$, the notation $a \lesssim b$ means that there exists a positive constant $c$ such that $a \leq c b$. We also denote $a \sim b$ when $a \lesssim b$ and $b \lesssim a$. Moreover, if $\alpha \in \mathbb{R}, \alpha_{+}$, respectively $\alpha_{-}$, will denote a number slightly greater, respectively lesser, than $\alpha$.

For $a_{1}, a_{2}, a_{3} \in \mathbb{R}$, it will be convenient to define the quantities $a_{\max } \geq a_{\text {med }} \geq$ $a_{\text {min }}$ to be the maximum, median and minimum of $a_{1}, a_{2}$ and $a_{3}$ respectively. For $a_{1}, a_{2}, a_{3}, a_{4} \in \mathbb{R}$, we define the quantities $a_{\max } \geq a_{s u b} \geq a_{t h d} \geq a_{\text {min }}$ to be

\footnotetext{
${ }^{6}$ This would be in contradiction with the $C^{2}$-ill-posedness results in 32 .
} 
the maximum, sub-maximum, third-maximum and minimum of $a_{1}, a_{2}, a_{3}$ and $a_{4}$ respectively. Usually, we use $k_{i}$ and $j_{i}$ to denote integers and $N_{i}=2^{k_{i}}, L_{i}=2^{j_{i}}$ to denote dyadic numbers.

For $u=u(x, t) \in \mathcal{S}\left(\mathbb{R}^{2}\right), \mathcal{F} u=\widehat{u}$ will denote its space-time Fourier transform, whereas $\mathcal{F}_{x} u=(u)^{\wedge_{x}}$, respectively $\mathcal{F}_{t} u=(u)^{\wedge_{t}}$, will denote its Fourier transform in space, respectively in time. Moreover, we generally omit the index $x$ or $t$ when the function depends only on one variable. For $s \in \mathbb{R}$, we define the Bessel and Riesz potentials of order $-s, J_{x}^{s}$ and $D_{x}^{s}$, by

$$
J_{x}^{s} u=\mathcal{F}_{x}^{-1}\left(\left(1+|\xi|^{2}\right)^{\frac{s}{2}} \mathcal{F}_{x} u\right) \quad \text { and } \quad D_{x}^{s} u=\mathcal{F}_{x}^{-1}\left(|\xi|^{s} \mathcal{F}_{x} u\right) .
$$

The unitary group $e^{t \partial_{x}^{5}}$ associated to the linear dispersive equation

$$
\partial_{t} u-\partial_{x}^{5} u=0
$$

is defined via Fourier transform by

$$
e^{t \partial_{x}^{5}} u_{0}=\mathcal{F}_{x}^{-1}\left(e^{i t w(\xi)} \mathcal{F}_{x} u_{0}\right),
$$

where $w(\xi)=\xi^{5}$.

For $k \in \mathbb{Z}_{+}$, let us define

$$
I_{k}=\left\{\xi \in \mathbb{R}: 2^{k-1} \leq|\xi| \leq 2^{k+1}\right\}
$$

if $k \geq 1$ and

$$
I_{0}=\{\xi \in \mathbb{R}:|\xi| \leq 2\} .
$$

Throughout the paper, we fix an even smooth cutoff function $\eta_{0}: \mathbb{R} \rightarrow[0,1]$ supported in $[-8 / 5,8 / 5]$ and such that $\eta_{0}$ is equal to 1 in $[-5 / 4,5 / 4]$. For $k \in$ $\mathbb{Z} \cap[1,+\infty)$, we define the functions $\eta_{k}$ and $\eta_{\leq k}$ respectively by

$$
\eta_{k}(\xi)=\eta_{0}\left(2^{-k} \xi\right)-\eta_{0}\left(2^{-(k-1)} \xi\right)=: \eta\left(2^{-k} \xi\right) \quad \text { and } \quad \eta_{\leq k}=\sum_{j=0}^{k} \eta_{j} .
$$

Then, $\left(\eta_{k}\right)_{k \geq 0}$ is a dyadic partition of the unity satisfying supp $\eta_{k} \subset I_{k}$.

Let $\left(\widetilde{\eta}_{k}\right)_{k \geq 0}$ be another nonhomogeneous dyadic partition of the unity satisfying $\operatorname{supp} \widetilde{\eta}_{k} \subset I_{k}$ and $\widetilde{\eta}_{k}=1$ on supp $\eta_{k}$.

Finally, for $k \in \mathbb{Z} \cap[1,+\infty)$, let us define the Fourier multipliers $P_{k}, P_{\leq 0}$ and $P_{\leq k}$ by

$$
P_{k} u=\mathcal{F}_{x}^{-1}\left(\eta_{k} \mathcal{F}_{x} u\right), P_{\leq 0} u=\mathcal{F}_{x}^{-1}\left(\eta_{0} \mathcal{F}_{x} u\right) \text {, and } P_{\leq k}=P_{\leq 0}+\sum_{j=1}^{k} P_{j} .
$$

Then it is clear that $P_{\leq 0}+\sum_{k=1}^{+\infty} P_{k}=1$. Often, when there is no risk of confusion, we also denote $P_{0}=P_{\leq 0}$.

2.2. Function spaces. For $1 \leq p \leq \infty, L^{p}(\mathbb{R})$ is the usual Lebesgue space with the norm $\|\cdot\|_{L^{p}}$, and for $s \in \mathbb{R}$, the Sobolev space $H^{s}(\mathbb{R})$ is defined via its usual norm $\|\phi\|_{H^{s}}=\left\|J_{x}^{s} \phi\right\|_{L^{2}}$.

Let $f=f(x, t)$ be a function defined for $x \in \mathbb{R}$ and $t$ in the time interval $[-T, T]$, with $T>0$ or in the whole line $\mathbb{R}$. Then if $X$ is one of the spaces defined above, we define the spaces $L_{T}^{p} X_{x}$ and $L_{t}^{p} X_{x}$ by the norms

$$
\|f\|_{L_{T}^{p} X_{x}}=\left(\int_{-T}^{T}\|f(\cdot, t)\|_{X}^{p} d t\right)^{\frac{1}{p}} \text { and }\|f\|_{L_{t}^{p} X_{x}}=\left(\int_{\mathbb{R}}\|f(\cdot, t)\|_{X}^{p} d t\right)^{\frac{1}{p}}
$$

when $1 \leq p<\infty$, with the natural modifications for $p=\infty$. 
We will work with the short-time localized Bourgain spaces introduced in 15 . First, for $k \in \mathbb{Z}_{+}$, we introduce the $l^{1}$-Besov type space $X_{k}$ of regularity $1 / 2$ with respect to modulations,

$$
X_{k}=\left\{\phi \in L^{2}\left(\mathbb{R}^{2}\right): \operatorname{supp} \phi \subset I_{k} \times \mathbb{R} \text { and }\|\phi\|_{X_{k}}<\infty\right\},
$$

where

$$
\|\phi\|_{X_{k}}=\sum_{j=0}^{+\infty} 2^{j / 2}\left\|\eta_{j}(\tau-w(\xi)) \phi(\xi, \tau)\right\|_{L_{\xi, \tau}^{2}} .
$$

Let $\alpha \geq 0$ be fixed. For $k \in \mathbb{Z}_{+}$, we introduce the space $F_{k, \alpha}$ possessing a $X_{k^{-}}$ structure in short-time intervals of length $2^{-\alpha k}$,

$$
F_{k, \alpha}=\left\{f \in L^{\infty}\left(\mathbb{R} ; L^{2}(\mathbb{R})\right): \operatorname{supp} \mathcal{F}(u) \subset I_{k} \times \mathbb{R} \text { and }\|f\|_{F_{k, \alpha}}<\infty\right\},
$$

where

$$
\|f\|_{F_{k, \alpha}}=\sup _{\tilde{t} \in \mathbb{R}}\left\|\mathcal{F}\left(\eta_{0}\left(2^{\alpha k}(\cdot-\tilde{t})\right) f\right)\right\|_{X_{k}} .
$$

Its dual version $N_{k, \alpha}$ is defined by

$$
N_{k, \alpha}=\left\{f \in L^{\infty}\left(\mathbb{R} ; H^{-1}(\mathbb{R})\right): \operatorname{supp} \mathcal{F}(f) \subset I_{k} \times \mathbb{R} \text { and }\|f\|_{N_{k, \alpha}}<\infty\right\},
$$

where

$$
\|f\|_{N_{k, \alpha}}=\sup _{\tilde{t} \in \mathbb{R}}\left\|\left(\tau-w(\xi)+i 2^{\alpha k}\right)^{-1} \mathcal{F}\left(\eta_{0}\left(2^{\alpha k}(\cdot-\tilde{t})\right) f\right)\right\|_{X_{k}} .
$$

Now for $s \in \mathbb{R}_{+}$, we define the global $F_{\alpha}^{s}$ and $N_{\alpha}^{s}$ spaces from their frequency localized versions $F_{k, \alpha}$ and $N_{k, \alpha}$ by using a nonhomogeneous Littlewood-Paley decomposition as follows:

$$
F_{\alpha}^{s}=\left\{f \in L^{\infty}\left(\mathbb{R} ; L^{2}(\mathbb{R})\right):\|f\|_{F_{\alpha}^{s}}=\left(\sum_{k=0}^{+\infty} 2^{2 k s}\left\|P_{k} f\right\|_{F_{k, \alpha}}^{2}\right)^{\frac{1}{2}}<\infty\right\}
$$

and

$$
N_{\alpha}^{s}=\left\{f \in L^{\infty}\left(\mathbb{R} ; H^{-1}(\mathbb{R})\right):\|f\|_{N_{\alpha}^{s}}=\left(\sum_{k=0}^{+\infty} 2^{2 k s}\left\|P_{k} f\right\|_{N_{k, \alpha}}^{2}\right)^{\frac{1}{2}}<\infty\right\} .
$$

We also define a localized (in time) version of those spaces. Let $T$ be a positive time and $Y$ denote $F_{\alpha}^{s}$ or $N_{\alpha}^{s}$. If $f: \mathbb{R} \times[-T, T] \rightarrow \mathbb{C}$, we define

$$
\|f\|_{Y(T)}=\inf \left\{\|\tilde{f}\|_{Y}: \tilde{f}: \mathbb{R}^{2} \rightarrow \mathbb{C} \text { and } \tilde{f}_{\mid \mathbb{R} \times[-T, T]}=f\right\} .
$$

Then,

and similarly

$$
F_{\alpha}^{s}(T)=\left\{f \in L^{\infty}\left([-T, T] ; L^{2}(\mathbb{R})\right):\|f\|_{F_{\alpha}^{s}(T)}<\infty\right\},
$$

$$
N_{\alpha}^{s}(T)=\left\{f \in L^{\infty}\left([-T, T] ; H^{-1}(\mathbb{R})\right):\|f\|_{N_{\alpha}^{s}(T)}<\infty\right\} .
$$

Finally for $s \in \mathbb{R}_{+}$and $T>0$, we define the energy space $B^{s}(T)$ by

$$
B^{s}(T)=\left\{f \in L^{\infty}\left([-T, T] ; L^{2}(\mathbb{R})\right):\|f\|_{B^{s}(T)}<\infty\right\},
$$


where

$$
\|f\|_{B^{s}(T)}=\left(\left\|P_{\leq 0} f(\cdot, 0)\right\|_{L^{2}}^{2}+\sum_{k=1}^{+\infty} 2^{2 k s} \sup _{t_{k} \in[-T, T]}\left\|P_{k} f\left(\cdot, t_{k}\right)\right\|_{L^{2}}^{2}\right)^{\frac{1}{2}}
$$

2.3. First properties. Following [15], we state some important properties of the $F_{\alpha}^{s}(T)$ spaces. First, we show that $F_{\alpha}^{s}(T) \hookrightarrow L^{\infty}\left([-T, T] ; H^{s}(\mathbb{R})\right)$.

Lemma 2.1. Let $T>0, s \in \mathbb{R}_{+}$and $\alpha \in \mathbb{R}_{+}$. Then it holds that

$$
\|f\|_{L_{T}^{\infty} H_{x}^{s}} \lesssim\|f\|_{F_{\alpha}^{s}(T)}
$$

for all $f \in F_{\alpha}^{s}(T)$.

Proof. Let $f \in F_{\alpha}^{s}(T)$. We choose $\tilde{f} \in F_{\alpha}^{s}$ such that

$$
\widetilde{f}_{\left.\right|_{[-T, T]}}=f \quad \text { and } \quad\|\widetilde{f}\|_{F_{\alpha}^{s}} \leq 2\|f\|_{F_{\alpha}^{s}(T)} .
$$

It follows that for every $t \in[-T, T]$,

$$
\|f(\cdot, t)\|_{H^{s}}=\|\widetilde{f}(\cdot, t)\|_{H^{s}} \lesssim\left(\sum_{k=0}^{+\infty} 2^{2 k s}\left\|\widetilde{f}_{k}(\cdot, t)\right\|_{L^{2}}^{2}\right)^{\frac{1}{2}}
$$

where $\widetilde{f}_{0}=P_{\leq 0} \tilde{f}$ and $\widetilde{f}_{k}=P_{k} \tilde{f}$ for any $k \in \mathbb{Z} \cap[1,+\infty)$.

Now fix $t \in[-T, T]$ and $k \in \mathbb{Z}_{+}$. The Fourier inversion formula gives that

$$
\mathcal{F}_{x}\left(\widetilde{f}_{k}\right)(\xi, t)=c \int_{\mathbb{R}} \mathcal{F}\left(\eta_{0}\left(2^{\alpha k}(\cdot-t)\right) \widetilde{f}_{k}\right)(\xi, \tau) e^{i t \tau} d \tau .
$$

On the other hand, the definition $X_{k}$ in (2.4) and the Cauchy-Schwarz inequality in $\tau$ implies that

$$
\left\|\int_{\mathbb{R}}|\phi(\xi, \tau)| d \tau\right\|_{L_{\xi}^{2}} \lesssim\|\phi\|_{X_{k}},
$$

for all $\phi \in X_{k}$. Therefore, it is deduced from (2.5), (2.13) and (2.14) that

$$
\left\|\widetilde{f}_{k}(\cdot, t)\right\|_{L^{2}} \lesssim\left\|\widetilde{f}_{k}\right\|_{F_{k, \alpha}},
$$

for all $k \in \mathbb{Z}_{+}$. Then, estimate (2.10) follows by gathering (2.11), (2.12), (2.15) and taking the supreme over $t \in[-T, T]$.

Then, we derive an important property involving the space $X_{k}$ (see [15]).

Lemma 2.2. Let $\alpha \geq 0$ and $l, k \in \mathbb{Z}_{+}$be given. Then, if $[\alpha l]$ denotes the integer part of $\alpha$, we have that

$$
2^{\frac{\alpha l}{2}}\left\|\eta_{\leq[\alpha l]}(\tau-w(\xi)) \int_{\mathbb{R}}\left|\phi\left(\xi, \tau^{\prime}\right)\right| 2^{-\alpha l}\left(1+2^{-\alpha l}\left|\tau-\tau^{\prime}\right|\right)^{-4} d \tau^{\prime}\right\|_{L_{\xi, \tau}^{2}} \lesssim\|\phi\|_{X_{k}}
$$

and

$$
\sum_{j>[\alpha l]} 2^{\frac{j}{2}}\left\|\eta_{j}(\tau-w(\xi)) \int_{\mathbb{R}}\left|\phi\left(\xi, \tau^{\prime}\right)\right| 2^{-\alpha l}\left(1+2^{-\alpha l}\left|\tau-\tau^{\prime}\right|\right)^{-4} d \tau^{\prime}\right\|_{L_{\xi, \tau}^{2}} \lesssim\|\phi\|_{X_{k}}
$$

for all $\phi \in X_{k}$. 
Proof. We fix $\tilde{l}=[\alpha l]$. We begin by proving estimate (2.16). Following [29], we use that $\left(\eta_{k}\right)_{k \geq 0}$ is the dyadic partition of the unity and the Cauchy-Schwarz inequality in $\tau^{\prime}$ to get that

$$
\begin{aligned}
I & :=2^{\frac{\alpha l}{2}}\left\|\eta_{\leq \tilde{l}}(\tau-w(\xi)) \int_{\mathbb{R}}\left|\phi\left(\xi, \tau^{\prime}\right)\right| 2^{-\alpha l}\left(1+2^{-\alpha l}\left|\tau-\tau^{\prime}\right|\right)^{-4} d \tau^{\prime}\right\|_{L_{\xi, \tau}^{2}} \\
& =2^{-\frac{\alpha l}{2}}\left\|\eta_{\leq \tilde{l}}(\tau-w(\xi)) \sum_{q=0}^{+\infty} \int_{\mathbb{R}} \eta_{q}\left(\tau^{\prime}-w(\xi)\right)\left|\phi\left(\xi, \tau^{\prime}\right)\right|\left(1+2^{-\alpha l}\left|\tau-\tau^{\prime}\right|\right)^{-4} d \tau^{\prime}\right\|_{L_{\xi, \tau}^{2}} \\
& \leq 2^{-\frac{\alpha l}{2}}\left\|\eta_{\leq \tilde{l}}(\tau-w(\xi)) \sum_{q=0}^{+\infty} I_{q, l}(\xi, \tau) 2^{\frac{q}{2}}\right\| \eta_{q}(\cdot-w(\xi)) \phi(\xi, \cdot)\left\|_{L^{2}}\right\|_{L_{\xi, \tau}^{2}},
\end{aligned}
$$

where

$$
I_{q, l}(\xi, \tau):=\left\|\widetilde{\eta}_{q}\left(\tau^{\prime}-w(\xi)\right)\left(1+2^{-\alpha l}\left|\tau-\tau^{\prime}\right|\right)^{-4}\left\langle\tau^{\prime}-w(\xi)\right\rangle^{-\frac{1}{2}}\right\|_{L_{\tau^{\prime}}^{2}} .
$$

Now, we get trivially that

$$
I_{q, l}(\xi, \tau) \lesssim 2^{\frac{q}{2}} 2^{-\frac{q}{2}} \lesssim 1,
$$

which concludes the proof of (2.16) recalling the definition of the space $X_{k}$ in (2.4).

Next, we turn to the proof of estimate (2.17). The mean value theorem yields

$$
\left|\eta_{j}(\tau-w(\xi))-\eta_{j}\left(\tau^{\prime}-w(\xi)\right)\right| \lesssim 2^{-j}\left|\tau-\tau^{\prime}\right|,
$$

which implies that

$$
\sum_{j>\tilde{l}} 2^{\frac{j}{2}}\left\|\eta_{j}(\tau-w(\xi)) \int_{\mathbb{R}}\left|\phi\left(\xi, \tau^{\prime}\right)\right| 2^{-\alpha l}\left(1+2^{-\alpha l}\left|\tau-\tau^{\prime}\right|\right)^{-4} d \tau^{\prime}\right\|_{L_{\xi, \tau}^{2}} \leq I I_{a}+I I_{b},
$$

where

$$
I I_{a}:=\sum_{j>\tilde{l}} 2^{\frac{j}{2}}\left\|\left[\eta_{j}(\cdot-w(\xi)) \phi(\xi, \cdot)\right] *\left[2^{-\alpha l}\left(1+2^{-\alpha l}|\cdot|\right)^{-4}\right](\tau)\right\|_{L_{\xi, \tau}^{2}}
$$

and

$$
I I_{b}:=\sum_{j>\tilde{l}} 2^{-\frac{j}{2}}\left\|\widetilde{\eta}_{j}(\tau-w(\xi)) \int_{\mathbb{R}}\left|\phi\left(\xi, \tau^{\prime}\right)\right| 2^{-\alpha l}\left|\tau-\tau^{\prime}\right|\left(1+2^{-\alpha l}\left|\tau-\tau^{\prime}\right|\right)^{-4} d \tau^{\prime}\right\|_{L_{\xi, \tau}^{2}} .
$$

Applying Young's theorem on convolution $\left(L_{\tau}^{2} * L_{\tau}^{1} \rightarrow L_{\tau}^{2}\right)$, we get that

$$
I I_{a} \lesssim \sum_{j>\tilde{l}} 2^{\frac{j}{2}}\left\|\eta_{j}(\tau-w(\xi)) \phi(\xi, \tau)\right\|_{L_{\xi, \tau}^{2}} \leq\|\phi\|_{X_{k}} .
$$

To deal with $I I_{b}$ we proceed as in the proof of estimate (2.16) and obtain that

$$
I I_{b} \lesssim 2^{-\tilde{l}} \sum_{j>\tilde{l}} 2^{-\frac{j}{2}}\left\|\widetilde{\eta}_{j}(\tau-w(\xi)) \sum_{q=0}^{+\infty} J_{q, l}(\xi, \tau) 2^{\frac{q}{2}}\right\| \eta_{q}(\cdot-w(\xi)) \phi(\xi, \cdot)\left\|_{L^{2}}\right\|_{L_{\xi, \tau}^{2}},
$$

where

$$
J_{q, l}(\xi, \tau):=\left\|\widetilde{\eta}_{q}\left(\tau^{\prime}-w(\xi)\right)\left|\tau-\tau^{\prime}\right|\left(1+2^{-\alpha l}\left|\tau-\tau^{\prime}\right|\right)^{-4}\left\langle\tau^{\prime}-w(\xi)\right\rangle^{-\frac{1}{2}}\right\|_{L_{\tau^{\prime}}^{2}} .
$$

In the case where $j \geq q+5$, we have that

$$
J_{q, l}(\xi, \tau) \lesssim 2^{j} 2^{-4(j-\tilde{l})}
$$


since $\left|\tau-\tau^{\prime}\right| \sim 2^{j}$. In the case where $q \geq j-4$, we get that

$$
J_{q, l}(\xi, \tau) \lesssim 2^{-\frac{j}{2}}\left\||\cdot|\left(1+2^{-\alpha l}|\cdot|\right)^{-4}\right\|_{L^{2}} \lesssim 2^{-\frac{j}{2}} 2^{\frac{3 \tilde{l}}{2}} .
$$

Then, after summing in $j$, we deduce that in both cases

$$
I I_{b} \lesssim \sum_{q=0}^{+\infty} 2^{\frac{q}{2}}\left\|\eta_{q}(\tau-w(\xi)) \phi(\xi, \tau)\right\|_{L_{\xi, \tau}^{2}}=\|\phi\|_{X_{k}} .
$$

Estimate (2.17) follows gathering (2.18) $-(2.20)$, which concludes the proof of Lemma 2.2 .

Corollary 2.3. Let $k \in \mathbb{Z}_{+}, \alpha \geq 0, \tilde{t} \in \mathbb{R}$ and $\gamma \in \mathcal{S}(\mathbb{R})$. Then it holds that

$$
\left\|\mathcal{F}\left[\gamma\left(2^{\alpha k}(\cdot-\tilde{t})\right) f\right]\right\|_{X_{k}} \lesssim\|\mathcal{F}(f)\|_{X_{k}},
$$

for all $f$ such that $\mathcal{F}(f) \in X_{k}$.

Proof. Since $\widehat{\gamma} \in \mathcal{S}\left(\mathbb{R}^{2}\right)$, we have that

$$
\begin{aligned}
\left|\mathcal{F}\left[\gamma\left(2^{\alpha k}(\cdot-\tilde{t})\right) f\right](\xi, \tau)\right| & =\left|\mathcal{F}(f)(\xi, \cdot) *\left[e^{-i \tilde{t}(\cdot)} 2^{-\alpha k} \widehat{\gamma}\left(2^{-\alpha k}(\cdot)\right)\right](\tau)\right| \\
& \leq \int_{\mathbb{R}}\left|\mathcal{F}(f)\left(\xi, \tau^{\prime}\right)\right| 2^{-\alpha k}\left(1+2^{-\alpha k}\left|\tau-\tau^{\prime}\right|\right)^{-4} d \tau^{\prime}
\end{aligned}
$$

Therefore estimate (2.21) follows by using the definition of $X_{k}$ and applying estimates (2.16) -2.17) to the right-hand side of (2.22).

Corollary 2.4. Let $k \in \mathbb{Z}_{+}, \alpha \geq 0, \tilde{t} \in \mathbb{R}$ and $\gamma \in \mathcal{S}(\mathbb{R})$. Then it holds that

$$
\left\|\left(\tau-w(\xi)+i 2^{\alpha k}\right)^{-1} \mathcal{F}\left[\gamma\left(2^{\alpha k}(\cdot-\tilde{t})\right) f\right]\right\|_{X_{k}} \lesssim\left\|\left(\tau-w(\xi)+i 2^{\alpha k}\right)^{-1} \mathcal{F}(f)\right\|_{X_{k}},
$$

for all $f$ such that $\mathcal{F}(f) \in X_{k}$.

Proof. We have that

$$
\begin{aligned}
\|(\tau-w(\xi)+ & \left.i 2^{\alpha k}\right)^{-1} \mathcal{F}\left[\gamma\left(2^{\alpha k}(\cdot-\tilde{t})\right) f\right] \|_{X_{k}} \\
\lesssim & 2^{-[\alpha k]} \sum_{j \leq[\alpha k]} 2^{\frac{j}{2}}\left\|\eta_{j}(\tau-w(\xi)) \mathcal{F}\left[\gamma\left(2^{\alpha k}(\cdot-\tilde{t})\right) f\right]\right\|_{L_{\xi, \tau}^{2}} \\
& +\sum_{j>[\alpha k]} 2^{-\frac{j}{2}}\left\|\eta_{j}(\tau-w(\xi)) \mathcal{F}\left[\gamma\left(2^{\alpha k}(\cdot-\tilde{t})\right) f\right]\right\|_{L_{\xi, \tau}^{2}} .
\end{aligned}
$$

We treat the first term on the right-hand side of (2.24) by using Lemma 2.2 as in the proof of Corollary 2.3 and the second term by using Lemma 2.2 and duality. This implies estimate (2.23).

Remark 2.5. For $s \in \mathbb{R}_{+}$, the classical dyadic Bourgain space $X^{s, \frac{1}{2}, 1}$ (introduced for instance in 35]) is defined by the norm

$$
\|f\|_{X^{s, \frac{1}{2}, 1}}=\left(\left\|\mathcal{F}\left(P_{\leq 0} f\right)\right\|_{X_{0}}^{2}+\sum_{k=1}^{+\infty} 2^{2 k s}\left\|\mathcal{F}\left(P_{k} f\right)\right\|_{X_{k}}^{2}\right)^{\frac{1}{2}} .
$$

Thus, if $f \in X^{s, \frac{1}{2}, 1}$, one may deduce after applying estimate (2.21) to each $P_{k} f$, taking the supreme in $\widetilde{t}$ and summing in $k$, that $\|f\|_{F_{\alpha}^{s}} \lesssim\|f\|_{X^{s, \frac{1}{2}, 1}}$, for any $\alpha \geq 0$. In other words, we have that

$$
X^{s, \frac{1}{2}, 1} \hookrightarrow F_{\alpha}^{s} \hookrightarrow L^{\infty}\left(\mathbb{R} ; H^{s}(\mathbb{R})\right) .
$$


More generally for any $k \in \mathbb{Z}_{+}$and $\alpha \geq 0$, we define the set $S_{k, \alpha}$ of $k$-acceptable time multiplication factors (cf. [15]) as

$$
S_{k, \alpha}=\left\{m_{k}: \mathbb{R} \rightarrow \mathbb{R}:\left\|m_{k}\right\|_{S_{k, \alpha}}=\sum_{j=0}^{10} 2^{-j \alpha k}\left\|\partial_{j} m_{k}\right\|_{L^{\infty}}<\infty\right\} .
$$

Corollary 2.6. Let $k \in \mathbb{Z}_{+}, \alpha \geq 0$ and $m_{k} \in S_{k, \alpha}$. Then it holds that

$$
\left\|m_{k} f\right\|_{F_{k, \alpha}} \lesssim\left\|m_{k}\right\|_{S_{k, \alpha}}\|f\|_{F_{k, \alpha}}
$$

and

$$
\left\|m_{k} f\right\|_{N_{k, \alpha}} \lesssim\left\|m_{k}\right\|_{S_{k, \alpha}}\|f\|_{N_{k, \alpha}} .
$$

Proof. We prove estimate (2.25). The proof of estimate (2.26) would follow in a similar way. Arguing as in the proof of Corollary 2.3 it suffices to prove that

$$
\left|\mathcal{F}_{t}\left[m_{k}(\cdot) \eta_{0}\left(2^{\alpha k}(\cdot-\tilde{t})\right)(\tau)\right]\right| \lesssim\left\|m_{k}\right\|_{S_{k, \alpha}} 2^{-\alpha k}\left(1+2^{-\alpha k}|\tau|\right)^{-4},
$$

for all $\tilde{t}, \tau \in \mathbb{R}$.

It follows from the definition of the Fourier transform that

$$
\begin{aligned}
\left\|\mathcal{F}_{t}\left[m_{k}(\cdot) \eta_{0}\left(2^{\alpha k}(\cdot-\tilde{t})\right)\right]\right\|_{L^{\infty}} & \lesssim\left\|m_{k}(\cdot) \eta_{0}\left(2^{\alpha k}(\cdot-\tilde{t})\right)\right\|_{L^{1}} \\
& \lesssim 2^{-\alpha k}\left\|m_{k}\right\|_{L^{\infty}}\left\|\eta_{0}\right\|_{L^{1}} .
\end{aligned}
$$

By again using basic properties of the Fourier transform and the Leibniz rule, we deduce that

$$
\begin{aligned}
2^{-4 \alpha k}|\tau|^{4} \mid \mathcal{F}_{t}\left[m_{k}(\cdot) \eta_{0}\right. & \left.\left(2^{\alpha k}(\cdot-\tilde{t})\right)\right](\tau) \mid \\
& \lesssim 2^{-4 \alpha k}\left\|\partial_{t}^{4}\left[m_{k}(\cdot) \eta_{0}\left(2^{\alpha k}(\cdot-\tilde{t})\right)\right]\right\|_{L^{1}} \\
& \lesssim 2^{-4 \alpha k} \sum_{j=0}^{4}\left\|\partial_{t}^{j} m_{k}\right\|_{L^{\infty}} 2^{(4-j) \alpha k} 2^{-\alpha k}\left\|\partial_{t}^{(4-j)} \eta_{0}\right\|_{L^{1}}
\end{aligned}
$$

Estimates (2.28)-(2.29) and the definition of $S_{k, \alpha}$ imply estimate (2.27), which concludes the proof of Corollary 2.6.

The next corollary of Lemma 2.2 will be useful in the proof of the bilinear and energy estimates (cf. Sections 4 and 5 ).

Corollary 2.7. Let $\alpha \geq 0, \tilde{t} \in \mathbb{R}$ and $l, k \in \mathbb{Z}_{+}$be such that $l+5 \geq k$. Then it holds that

$$
2^{\frac{\alpha l}{2}}\left\|\eta_{\leq[\alpha l]}(\tau-w(\xi)) \mathcal{F}\left[\eta_{0}\left(2^{\alpha l}(\cdot-\tilde{t})\right) f\right]\right\|_{L_{\xi, \tau}^{2}} \lesssim\|f\|_{F_{k, \alpha}}
$$

and

$$
\sum_{j>[\alpha l]} 2^{\frac{j}{2}}\left\|\eta_{j}(\tau-w(\xi)) \mathcal{F}\left[\eta_{0}\left(2^{\alpha l}(\cdot-\tilde{t})\right) f\right]\right\|_{L_{\xi, \tau}^{2}} \lesssim\|f\|_{F_{k, \alpha}},
$$

for all $f \in F_{k, \alpha}$.

Proof. Observe that

$$
\eta_{0}\left(2^{\alpha l}(\cdot-\tilde{t})\right) f=\eta_{0}\left(2^{\alpha l}(\cdot-\tilde{t})\right) \eta_{0}\left(2^{\alpha(k-6)}(\cdot-\tilde{t})\right) f .
$$

Moreover, it follows from Corollary 2.6 that

$$
\left\|\mathcal{F}\left[\eta_{0}\left(2^{\alpha(k-6)}(\cdot-\tilde{t})\right) f\right]\right\|_{X^{k}} \lesssim\|f\|_{F_{k, \alpha}} .
$$

Therefore, we conclude estimates (2.30) and (2.31) by applying (2.16) and (2.17), arguing as was done in the proof of Corollary 2.3 . 
Remark 2.8. Estimate (2.30) can be viewed as a consequence of the uncertainty Heisenberg principle. It is of fundamental importance in the proof of the short-time bilinear estimates (cf. Section 44), since it allows us to consider only regions where the modulation $|\tau-w(\xi)|$ is not too small, and therefore to avoid the regions giving trouble in the low-high frequency interactions (cf. [32]).

2.4. Linear estimates. In this subsection, we derive the linear estimate associated to the spaces $F_{\alpha}^{s}(T)$ (cf. [15]).

Proposition 2.9. Assume $s \in \mathbb{R}_{+}, \alpha>0$ and $T \in(0,1]$. Then we have that

$$
\|u\|_{F_{\alpha}^{s}(T)} \lesssim\|u\|_{B^{s}(T)}+\|f\|_{N_{\alpha}^{s}(T)},
$$

for all $u \in B^{s}(T)$ and $f \in N_{\alpha}^{s}$ satisfying

$$
\partial_{t} u-\partial_{x}^{5} u=f, \quad \text { on } \quad \mathbb{R} \times[-T, T] .
$$

Remark 2.10. Observe that when working in the classical Bourgain space $X^{0, \frac{1}{2}, 1}(T)$ defined in Remark 2.5, one would obtain an estimate of the form

$$
\|u\|_{X^{s, \frac{1}{2}, 1}(T)} \lesssim\|u(0)\|_{H^{s}}+\|f\|_{X^{s,-\frac{1}{2}, 1}(T)} .
$$

Here, we need to introduce the energy norm $\|u\|_{B^{s}(T)}$ instead of $\|u(0)\|_{H^{s}}$, since we are working on very short time intervals whose lengths depend on the spatial frequency.

We first derive a homogeneous and a nonhomogeneous linear estimate in the spaces $X_{k}$.

Lemma 2.11 (Homogeneous linear estimate). Let $\alpha \geq 0$ and $k \in \mathbb{Z}_{+}$. Then it holds that

$$
\left\|\mathcal{F}\left[\eta_{0}\left(2^{\alpha k} t\right) e^{t \partial_{x}^{5}} u_{0}\right]\right\|_{X_{k}} \lesssim\left\|u_{0}\right\|_{L^{2}},
$$

for all $u_{0} \in L^{2}(\mathbb{R})$ such that $\operatorname{supp} \mathcal{F}_{x}\left(u_{0}\right) \in I_{k}$.

Proof. A direct computation shows that

$$
\mathcal{F}\left[\eta_{0}\left(2^{\alpha k} t\right) e^{t \partial_{x}^{5}} u_{0}\right](\xi, \tau)=2^{-\alpha k} \widehat{\eta}_{0}\left(2^{-\alpha k}(\tau-w(\xi))\right) \widehat{u}_{0}(\xi) .
$$

Thus, it follows from the definition of $X_{k}$ and Plancherel's identity that

$$
\left\|\mathcal{F}\left[\eta_{0}\left(2^{\alpha k} t\right) e^{t \partial_{x}^{5}} u_{0}\right]\right\|_{X_{k}} \leq \sum_{j \geq 0} 2^{j / 2}\left\|\eta_{j}(\cdot) 2^{-\alpha k} \widehat{\eta}_{0}\left(2^{-\alpha k} \cdot\right)\right\|_{L^{2}}\left\|u_{0}\right\|_{L^{2}} .
$$

Moreover, it is clear since $\widehat{\eta}_{0} \in \mathcal{S}(\mathbb{R})$ that

$$
\begin{aligned}
\left\|\eta_{j}(\cdot) 2^{-\alpha k} \widehat{\eta}_{0}\left(2^{-\alpha k} \cdot\right)\right\|_{L^{2}} & \lesssim 2^{-\alpha k}\left\|\eta_{j}(\cdot)\left(1+2^{-\alpha k}|\cdot|\right)^{-4}\right\|_{L^{2}} \\
& \lesssim 2^{-\alpha k} 2^{j / 2} \min \left(1,2^{4(\alpha k-j)}\right),
\end{aligned}
$$

which combined with (2.35) implies estimate (2.34).

Lemma 2.12 (Nonhomogeneous linear estimate). Let $\alpha \geq 0$ and $k \in \mathbb{Z}_{+}$. Then it holds that

$$
\left\|\mathcal{F}\left[\eta_{0}\left(2^{\alpha k} t\right) \int_{0}^{t} e^{(t-s) \partial_{x}^{5}} f(\cdot, s) d s\right]\right\|_{X_{k}} \lesssim\left\|\left(\tau-w(\xi)+i 2^{\alpha k}\right)^{-1} \mathcal{F}(f)\right\|_{X_{k}},
$$

for all $f$ such that supp $\mathcal{F}(f) \in I_{k} \times \mathbb{R}$. 
Proof. Straightforward computations yield

$$
\begin{aligned}
& \mathcal{F}\left[\eta_{0}\left(2^{\alpha k} t\right) \int_{0}^{t} e^{(t-s) \partial_{x}^{5}} f(\cdot, s) d s\right](\xi, \tau) \\
& =\mathcal{F}_{t}\left[\eta_{0}\left(2^{\alpha k} t\right) \int_{\mathbb{R}} \frac{e^{i t \tilde{\tau}}-e^{i t w(\xi)}}{i(\tilde{\tau}-w(\xi))} \mathcal{F}(f)(\xi, \tilde{\tau}) d \tilde{\tau}\right](\tau) \\
& =2^{-\alpha k} \widehat{\eta}_{0}\left(2^{-\alpha k} \cdot\right) *\left[\frac{\mathcal{F}(f)(\xi, \cdot)}{i(\cdot-w(\xi))}\right](\tau)-\mathcal{F}_{t}\left[\eta_{0}\left(2^{\alpha k} t\right) e^{i t w(\xi)}\right](\tau) \int_{\mathbb{R}} \frac{\mathcal{F}(f)(\xi, \tilde{\tau})}{i(\tilde{\tau}-w(\xi))} d \tilde{\tau} \\
& =2^{-\alpha k} \int_{\mathbb{R}} \frac{\widehat{\eta}_{0}\left(2^{-\alpha k}(\tau-\tilde{\tau})\right)-\widehat{\eta}_{0}\left(2^{-\alpha k}(\tau-w(\xi))\right)}{i(\tilde{\tau}-w(\xi))} \mathcal{F}(f)(\xi, \tilde{\tau}) d \tilde{\tau} .
\end{aligned}
$$

Now, we observe that

$$
\begin{aligned}
2^{-\alpha k} \mid & \frac{\widehat{\eta}_{0}\left(2^{-\alpha k}(\tau-\tilde{\tau})\right)-\widehat{\eta}_{0}\left(2^{-\alpha k}(\tau-w(\xi))\right)}{i(\tilde{\tau}-w(\xi))}\left(\tilde{\tau}-w(\xi)+i 2^{\alpha k}\right) \mid \\
& \lesssim 2^{-\alpha k}\left(1+2^{-\alpha k}|\tau-\tilde{\tau}|\right)^{-4}+2^{-\alpha k}\left(1+2^{-\alpha k}|\tau-w(\xi)|\right)^{-4}
\end{aligned}
$$

Indeed, in the case where $|\tilde{\tau}-w(\xi)| \geq 2^{\alpha k}$, then $\left|\tilde{\tau}-w(\xi)+i 2^{\alpha k}\right| \lesssim|\tilde{\tau}-w(\xi)|$ and estimate (2.38) follows directly from the fact that $\widehat{\eta}_{0} \in \mathcal{S}(\mathbb{R})$ and the triangle inequality. Now we deal with the case where $|\tilde{\tau}-w(\xi)| \leq 2^{\alpha k}$. We deduce by applying the mean value theorem to the even function $\widehat{\eta}_{0}$ that

$$
\left|\widehat{\eta}_{0}\left(2^{-\alpha k}(\tau-\tilde{\tau})\right)-\widehat{\eta}_{0}\left(2^{-\alpha k}(\tau-w(\xi))\right)\right| \leq 2^{-\alpha k}\left|\widehat{\eta}_{0}^{\prime}\left(2^{-\alpha k} \theta\right)\right||\tilde{\tau}-w(\xi)|,
$$

for some $\theta \in]|\tau-\tilde{\tau}|,|\tau-w(\xi)|[$ or $\theta \in]|\tau-w(\xi)|,|\tau-\tilde{\tau}|[$, depending on whether $|\tau-\tilde{\tau}|<|\tau-w(\xi)|$ or $|\tau-w(\xi)|<|\tau-\tilde{\tau}|$. Thus, since $\widehat{\eta}_{0}^{\prime} \in \mathcal{S}(\mathbb{R})$, the left-hand side of (2.38) can be bounded by $2^{-\alpha k}\left(1+2^{-\alpha k}|\theta|\right)^{-4}$. This implies estimate (2.38) in this case by using the assumption on $\theta$.

On the one hand, we deduce from Lemma 2.2 that

$$
\left\|\int_{\mathbb{R}} \frac{|\mathcal{F}(f)(\xi, \tilde{\tau})|}{\left|\tilde{\tau}-w(\xi)+i 2^{\alpha k}\right|} 2^{-\alpha k}\left(1+2^{-\alpha k}|\tau-\tilde{\tau}|\right)^{-4} d \tilde{\tau}\right\|_{X_{k}} \lesssim\left\|\frac{\mathcal{F}(f)(\xi, \tau)}{\tau-w(\xi)+i 2^{\alpha k}}\right\|_{X_{k}} .
$$

On the other hand, it follows, arguing as in the proof of Lemma 2.11 and using estimate (2.14), that

$$
\begin{aligned}
& \left\|2^{-\alpha k}\left(1+2^{-\alpha k}|\tau-w(\xi)|\right)^{-4} \int_{\mathbb{R}} \frac{|\mathcal{F}(f)(\xi, \tilde{\tau})|}{\left|\tilde{\tau}-w(\xi)+i 2^{\alpha k}\right|} d \tilde{\tau}\right\|_{X_{k}} \\
& \lesssim \sum_{j \geq 0} 2^{j / 2}\left\|\eta_{j}(\cdot) 2^{-\alpha k}\left(1+2^{-\alpha k}|\cdot|\right)^{-4}\right\|_{L^{2}}\left\|\int_{\mathbb{R}} \frac{|\mathcal{F}(f)(\xi, \tilde{\tau})|}{\left|\tilde{\tau}-w(\xi)+i 2^{\alpha k}\right|} d \tilde{\tau}\right\|_{L_{\xi}^{2}} \\
& \lesssim \sum_{j \geq 0} 2^{j} 2^{-\alpha k} \min \left(1,2^{4(\alpha k-j)}\right)\left\|\int_{\mathbb{R}} \frac{|\mathcal{F}(f)(\xi, \tilde{\tau})|}{\left|\tilde{\tau}-w(\xi)+i 2^{\alpha k}\right|} d \tilde{\tau}\right\|_{L_{\xi}^{2}} \\
& \lesssim\left\|\frac{\mathcal{F}(f)(\xi, \tau)}{\tau-w(\xi)+i 2^{\alpha k}}\right\|_{X_{k}} .
\end{aligned}
$$

Finally, we conclude the proof of Proposition 2.12 by gathering (2.37)-(2.40).

A proof of Proposition 2.9 is now in sight. 
Proof of Proposition 2.9. Let $u, f: \mathbb{R} \times[-T, T]$ satisfy (2.33). First, we choose an extension $\tilde{f}$ of $f$ on $\mathbb{R}^{2}$ satisfying

$$
\|\widetilde{f}\|_{N_{\alpha}^{s}} \leq 2\|f\|_{N_{\alpha}^{s}(T)} .
$$

Fix $\theta \in C_{0}^{\infty}(\mathbb{R})$ such that $\theta(t)=1$ if $t \geq 1$ and $\theta(t)=0$ if $t \leq 0$. For $k \in \mathbb{Z}_{+}$, we define

$$
\widetilde{f}_{k}=\theta\left(2^{\alpha k+10}\left(t+T+2^{-\alpha k-10}\right)\right) \theta\left(-2^{\alpha k+10}\left(t-T-2^{-\alpha k-10}\right)\right) P_{k} \tilde{f} .
$$

Then, it follows from (2.26) and the definition of $\theta$ that

$$
\left\|\widetilde{f}_{k}\right\|_{N_{k, \alpha}} \lesssim\left\|P_{k} \widetilde{f}\right\|_{N_{k, \alpha}}
$$

$$
\operatorname{supp} \tilde{f}_{k} \subset \mathbb{R} \times\left[-T-2^{-\alpha k-10}, T+2^{-\alpha k-10}\right] \quad \text { and } \quad \tilde{f}_{\left.k\right|_{[-T, T]}}=P_{k} f .
$$

Moreover, for all $k \in \mathbb{Z}_{+}$, we also extend $P_{k} u$ on $\mathbb{R}^{2}$ by defining $\widetilde{u}_{k}(t)$ as

$$
\left\{\begin{array}{cl}
\eta_{0}\left(2^{\alpha k+5}(t-T)\right)\left(e^{(t-T) \partial_{x}^{5}} P_{k} u(T)+\int_{T}^{t} e^{(t-s) \partial_{x}^{5}} \widetilde{f}_{k}(s) d s\right) & \text { if } t>T, \\
P_{k} u(t) & \text { if } t \in[-T, T], \\
\eta_{0}\left(2^{\alpha k+5}(t+T)\right)\left(e^{(t+T) \partial_{x}^{5}} P_{k} u(-T)+\int_{-T}^{t} e^{(t-s) \partial_{x}^{5}} \widetilde{f}_{k}(s) d s\right) & \text { if } t<-T .
\end{array}\right.
$$

Next, we show that

$$
\left\|\widetilde{u}_{k}\right\|_{F_{k, \alpha}} \lesssim \sup _{t_{k} \in[-T, T]}\left\|\mathcal{F}\left(\eta_{0}\left(2^{\alpha k}\left(t-t_{k}\right)\right) \widetilde{u}_{k}\right)\right\|_{X_{k}} .
$$

It is clear from the definition that $\widetilde{u}_{k}$ is supported in $\mathbb{R} \times\left[T-2^{-\alpha k-5}, T+2^{-\alpha k-5}\right]$. Thus, if $t_{k}>T$, we get

$$
\eta_{0}\left(2^{\alpha k}\left(t-t_{k}\right)\right) \widetilde{u}_{k}=\eta_{0}\left(2^{\alpha k}\left(t-t_{k}\right)\right) \eta_{0}\left(2^{\alpha k}\left(t-\tilde{t}_{k}\right)\right) \widetilde{u}_{k}
$$

for some $\tilde{t}_{k} \in\left[T-2^{-\alpha k}, T\right]$, so that (2.21) implies

$$
\sup _{t_{k}>T}\left\|\mathcal{F}\left[\eta_{0}\left(2^{\alpha k}\left(\cdot-t_{k}\right)\right) \widetilde{u}_{k}\right]\right\|_{X_{k}} \lesssim \sup _{\tilde{t}_{k} \in[-T, T]}\left\|\mathcal{F}\left[\eta_{0}\left(2^{\alpha k}\left(\cdot-\tilde{t}_{k}\right)\right) \widetilde{u}_{k}\right]\right\|_{X_{k}} \cdot
$$

We could argue similarly for $t<T$, which implies estimate (2.43).

Now we fix $t_{k} \in[-T, T]$. Observe that

$$
\left\|\mathcal{F}\left[\eta_{0}\left(2^{\alpha k}\left(\cdot-t_{k}\right)\right) \widetilde{u}_{k}\right]\right\|_{X_{k}}=\left\|\mathcal{F}\left[\eta_{0}\left(2^{\alpha k} \cdot\right) \widetilde{u}_{k}\left(\cdot+t_{k}\right)\right]\right\|_{X_{k}}
$$

and by the Duhamel principle that

$\eta_{0}\left(2^{\alpha k} t\right) \widetilde{u}_{k}\left(t+t_{k}\right)=m_{k}(t) \eta_{0}\left(2^{\alpha k} t\right)\left(e^{i t \partial_{x}^{5}} P_{k} u\left(t_{k}\right)+\int_{0}^{t} e^{i(t-s) \partial_{x}^{5}} \widetilde{\eta}_{0}\left(2^{\alpha k} s\right) \widetilde{f}_{k}\left(s+t_{k}\right) d s\right)$,

where $m_{k} \in S_{k, \alpha}$. Thus, we deduce from estimates (2.25), (2.34) and (2.36) that

$$
\begin{aligned}
& \left\|\mathcal{F}\left[\eta_{0}\left(2^{\alpha k} \cdot\right) \widetilde{u}_{k}\left(\cdot+t_{k}\right)\right]\right\|_{X_{k}} \\
& \quad \lesssim\left\|P_{k} u\left(t_{k}\right)\right\|_{L^{2}}+\left\|\left(\tau-w(\xi)+i 2^{\alpha k}\right)^{-1} \mathcal{F}\left(\widetilde{\eta}_{0}\left(2^{\alpha k} \cdot\right) \widetilde{f}_{k}\left(\cdot+t_{k}\right)\right)\right\|_{X_{k}} .
\end{aligned}
$$

Finally, we define $\widetilde{u}=\sum_{k \in \mathbb{Z}_{+}} \widetilde{u}_{k}$. Then, it is clear that $\widetilde{u}$ extends $u$ outside of $[-T, T]$. Moreover, arguing as in the proof of Lemma 6.3, we get that

$$
\left\|P_{k} \widetilde{u}\right\|_{F_{k}} \lesssim \sum_{\left|k-k^{\prime}\right| \leq 3, k^{\prime} \in \mathbb{Z}_{+}}\left\|\widetilde{u}_{k^{\prime}}\right\|_{F_{k^{\prime}}} .
$$

Therefore, we conclude the proof of estimate (2.32) by gathering (2.26), (2.41) (2.45), taking the supreme in $t_{k} \in[-T, T]$ and summing over $k \in \mathbb{Z}_{+}$. 
2.5. Strichartz estimates. We recall the Strichartz estimates associated to $\left\{e^{t \partial_{x}^{5}}\right\}$ proved by Kenig, Ponce and Vega in [19.

Proposition 2.13. Let $2 \leq q, r \leq+\infty$ and $0 \leq s \leq \frac{3}{q}$ satisfy $-s+\frac{5}{q}+\frac{1}{r}=\frac{1}{2}$. Then,

$$
\left\|D_{x}^{s} e^{t \partial_{x}^{5}} u_{0}\right\|_{L_{t}^{q} L_{x}^{r}} \lesssim\left\|u_{0}\right\|_{L^{2}}
$$

for all $u_{0} \in L^{2}(\mathbb{R})$.

As a consequence, we obtain a Strichartz estimate in the context of the Bourgain spaces $F_{\alpha}^{s}(T)$.

Corollary 2.14. Assume $0<T \leq 1, \alpha \geq 0$ and $\epsilon>0$. Then, it holds that

$$
\left\|D_{x}^{\frac{3}{4}-\frac{\alpha}{4}} u\right\|_{L_{T}^{2} L_{x}^{\infty}} \lesssim\|u\|_{F_{\alpha}^{\epsilon}(T)}
$$

and

$$
\left\|D_{x}^{\frac{3}{4}-\frac{\alpha}{4}} u\right\|_{L_{T}^{2} L_{x}^{\infty}}:=\left(\sum_{k \geq 0}\left\|D_{x}^{\frac{3}{4}-\frac{\alpha}{4}} P_{k} u\right\|_{L_{T}^{2} L_{x}^{\infty}}^{2}\right)^{\frac{1}{2}} \lesssim\|u\|_{F_{\alpha}^{\epsilon}(T)},
$$

for any $u \in F_{\alpha}^{\epsilon}(T)$.

Proof. Let $0<T \leq 1, \alpha \geq 0, \epsilon>0$ and $u \in F_{\alpha}^{\epsilon}(T)$. Choose $\widetilde{u} \in F_{\alpha}^{\epsilon}$ such that

$$
\widetilde{u}_{\left.\right|_{[-T, T]}}=u \quad \text { and } \quad\|\widetilde{u}\|_{F_{\alpha}^{\epsilon}} \leq 2\|u\|_{F_{\alpha}^{\epsilon}(T)} .
$$

For $k \in \mathbb{Z}_{+}$, we denote $\widetilde{u}_{k}=P_{k} \widetilde{u}$ (recall that $P_{0}=P_{\leq 0}$ ). Then we deduce using the Sobolev embedding $W^{\epsilon^{\prime}, r}(\mathbb{R}) \hookrightarrow L^{\infty}(\mathbb{R})$, the square function theorem and Minkowski's inequality that

$$
\left\|D_{x}^{\frac{3}{4}} u\right\|_{L_{T}^{2} L_{x}^{\infty}} \lesssim\left\|D_{x}^{\frac{3}{4}} J_{x}^{\epsilon^{\prime}} \widetilde{u}\right\|_{L_{T}^{2} L_{x}^{r}} \lesssim\left(\sum_{k \geq 0} 2^{2 k \epsilon^{\prime}}\left\|D_{x}^{\frac{3}{4}} \widetilde{u}_{k}\right\|_{L_{T}^{2} L_{x}^{r}}^{2}\right)^{\frac{1}{2}},
$$

where $\epsilon^{\prime}$ and $r\left(>1 / \epsilon^{\prime}\right)$ will be chosen later. Therefore, according to the definition of $F_{\alpha}^{\epsilon}$ in (2.7), it suffices to prove that

$$
\left\|D_{x}^{\frac{3}{4}} \widetilde{u}_{k}\right\|_{L_{T}^{2} L_{x}^{r}} \lesssim 2^{k\left(\frac{\alpha}{4}+\frac{3-\alpha}{2 r}\right)}\left\|\widetilde{u}_{k}\right\|_{F_{k, \alpha}},
$$

for all $k \geq 0$ in order to prove estimate (2.47). Indeed, it is enough then to choose $r$ and $\epsilon^{\prime}$ such that $r \epsilon^{\prime}>1$ and $\epsilon^{\prime}+\frac{3-\alpha}{2 r}<\epsilon$.

Next, we prove estimate (2.49). For $k \geq 0$, we chop the interval $[-T, T]$ into subintervals $I_{j}$ of length $2^{-\alpha k}$. Let $[-T, T]=U_{j} I_{j}$ where $\left|I_{j}\right| \sim 2^{-\alpha k}$ and $\eta_{0}\left(2^{\alpha k}\left(\cdot-c_{j}\right)\right)=1$ on $I_{j}$ (here $c_{j}$ denotes the center of $I_{j}$ ). Note that the number of intervals $I_{j}$ is of order $2^{\alpha k} T$. Let $2 \leq q$ be so that $-\frac{3}{q}+\frac{5}{q}+\frac{1}{r}=\frac{1}{2}$. Then, we deduce, applying Hölder's inequality in time, that

$$
\begin{aligned}
\left\|D_{x}^{\frac{3}{4}} \widetilde{u}_{k}\right\|_{L_{T}^{2} L_{x}^{r}} & =\left(\sum_{j}\left\|D_{x}^{\frac{3}{4}} \widetilde{u}_{k} \eta_{0}\left(2^{\alpha k}\left(\cdot-c_{j}\right)\right)\right\|_{L_{I_{j}}^{2} L_{x}^{r}}^{2}\right)^{\frac{1}{2}} \\
& \lesssim 2^{-\alpha k\left(\frac{1}{2}-\frac{1}{q}\right)}\left(\sum_{j}\left\|D_{x}^{\frac{3}{4}} \widetilde{u}_{k} \eta_{0}\left(2^{\alpha k}\left(\cdot-c_{j}\right)\right)\right\|_{L_{I_{j}}^{q} L_{x}^{r}}^{2}\right)^{\frac{1}{2}} .
\end{aligned}
$$

Due to the Fourier inversion formula, we have that

$$
D_{x}^{\frac{3}{4}} \widetilde{u}_{k}(x, t) \eta_{0}\left(2^{\alpha k}\left(t-c_{j}\right)\right)=c \int_{\mathbb{R}} D_{x}^{\frac{3}{4}} e^{t \partial_{x}^{5}} \mathcal{F}_{s}\left(e^{-s \partial_{x}^{5}} \eta_{0}\left(2^{\alpha k}\left(s-c_{j}\right)\right) \widetilde{u}_{k}(\cdot, s)\right)(\tau) e^{i t \tau} d \tau .
$$


Thus, Minkowski's inequality, estimate (2.46), Plancherel's identity and the CauchySchwarz inequality in $q$ imply that

$$
\begin{aligned}
& \left\|D_{x}^{\frac{3}{4}} \widetilde{u}_{k}\right\|_{L_{I_{j}}^{q} L_{x}^{r}} \lesssim \int_{\mathbb{R}}\left\|D_{x}^{\frac{3}{4}} e^{t \partial_{x}^{5}} \mathcal{F}_{s}\left(e^{-s \partial_{x}^{5}} \eta_{0}\left(2^{\alpha k}\left(s-c_{j}\right)\right) \widetilde{u}_{k}\right)\right\|_{L_{I_{j}}^{q} L_{x}^{r}} d \tau \\
& \lesssim 2^{k\left(\frac{3}{4}-\frac{3}{q}\right)} \sum_{q \geq 0} 2^{q / 2}\left\|\eta_{q}(\tau) \mathcal{F}\left(e^{-\cdot \partial_{x}^{5}} \eta_{0}\left(2^{\alpha k}\left(\cdot-c_{j}\right)\right) \widetilde{u}_{k}\right)(\xi, \tau)\right\|_{L_{\xi, \tau}^{2}} .
\end{aligned}
$$

Then, we observe that

$$
\mathcal{F}\left(e^{-\cdot \partial_{x}^{5}} \eta_{0}\left(2^{\alpha k}\left(\cdot-c_{j}\right)\right) \widetilde{u}_{k}\right)(\xi, \tau)=\mathcal{F}\left(\eta_{0}\left(2^{\alpha k}\left(\cdot-c_{j}\right)\right) \widetilde{u}_{k}\right)(\xi, \tau+w(\xi)),
$$

which together with (2.51) and the definition of $F_{k, \alpha}$ in (2.5) implies that

$$
\left\|D_{x}^{\frac{3}{4}} \widetilde{u}_{k}\right\|_{L_{I_{j}}^{q} L_{x}^{r}} \lesssim 2^{k\left(\frac{3}{4}-\frac{3}{q}\right)}\left\|\widetilde{u}_{k}\right\|_{F_{k, \alpha}} .
$$

Finally, we deduce by combining (2.50) and (2.52) that

$$
\left\|D_{x}^{\frac{3}{4}} \widetilde{u}_{k}\right\|_{L_{T}^{2} L_{x}^{r}} \lesssim 2^{k\left(\frac{\alpha}{q}+\frac{3}{4}-\frac{3}{q}\right)}\left\|\widetilde{u}_{k}\right\|_{F_{k, \alpha}},
$$

which yields estimate (2.49) since $\frac{1}{q}=\frac{1}{4}-\frac{1}{2 r}$. The proof of estimate (2.48) is similar.

Next, we derive a bilinear Strichartz estimate for the group $\left\{e^{t \partial_{x}^{5}}\right\}$, which is an extension of the one proved in [10] for the Airy equation (see also Lemma 3.4 in [14] for the dispersion generalized Benjamin-Ono equation). Let $\zeta \in C^{\infty}$ be an even function such that $\zeta_{\left.\right|_{[-1,1]}}=0, \zeta_{\left.\right|_{\mathbb{R} \backslash[-2,2]}}=1$ and $0 \leq \zeta \leq 1$. We define $|x|_{1}=\zeta(x)|x|$.

Lemma 2.15. For $s \in \mathbb{R}$, we define the bilinear operator $\mathcal{J}^{s}$ by

$$
\mathcal{F}_{x}\left(\mathcal{J}^{s}\left(u_{1}, u_{2}\right)\right)(\xi)=\left.\int_{\xi=\xi_{1}+\xi_{2}}|| \xi_{1}\right|^{2 s}-\left.\left|\xi_{2}\right|^{2 s}\right|_{1} ^{\frac{1}{2}} \widehat{u}_{1}\left(\xi_{1}\right) \widehat{u}_{2}\left(\xi_{2}\right) d \xi_{1} .
$$

Then, it holds that

$$
\left\|\mathcal{J}^{2}\left(e^{t \partial_{x}^{5}} u_{1}, e^{t \partial_{x}^{5}} u_{2}\right)\right\|_{L_{x, t}^{2}} \lesssim\left\|u_{1}\right\|_{L^{2}}\left\|u_{2}\right\|_{L^{2}},
$$

for any $u_{1}, u_{2} \in L^{2}(\mathbb{R})$.

Proof. For a fixed $t \in \mathbb{R}$, we get by using Plancherel's identity that

$$
\begin{aligned}
\left\|\mathcal{J}^{2}\left(e^{t \partial_{x}^{5}} u_{1}, e^{t \partial_{x}^{5}} u_{2}\right)\right\|_{L_{x}^{2}}^{2} & \\
& =\int_{\mathbb{R}}\left|\int_{\xi=\xi_{1}+\xi_{2}}\right|\left|\xi_{1}\right|^{4}-\left.\left.\left|\xi_{2}\right|^{4}\right|_{1} ^{\frac{1}{2}} e^{i t\left(\xi_{1}^{5}+\xi_{2}^{5}\right)} \widehat{u}_{1}\left(\xi_{1}\right) \widehat{u}_{2}\left(\xi_{2}\right) d \xi_{1}\right|^{2} d \xi \\
& =\int_{\mathbb{R}^{3}} e^{i t \phi\left(\xi, \xi_{1}, \eta_{1}\right)} f\left(\xi, \xi_{1}, \eta_{1}\right) d \xi d \xi_{1} d \eta_{1},
\end{aligned}
$$

where the phase function $\phi$ is given by

$$
\phi\left(\xi, \xi_{1}, \eta_{1}\right):=\xi_{1}^{5}+\left(\xi-\xi_{1}\right)^{5}-\eta_{1}^{5}-\left(\xi-\eta_{1}\right)^{5},
$$

and $f$ is defined by

$$
\begin{aligned}
& f\left(\xi, \xi_{1}, \eta_{1}\right) \\
& \quad:=\left.|| \xi_{1}\right|^{4}-\left.\left.\left|\xi-\xi_{1}\right|^{4}\right|_{1} ^{\frac{1}{2}}|| \eta_{1}\right|^{4}-\left.\left|\xi-\eta_{1}\right|^{4}\right|_{1} ^{\frac{1}{2}} \widehat{u}_{1}\left(\xi_{1}\right) \widehat{u}_{2}\left(\xi-\xi_{1}\right) \overline{\widehat{u}_{1}\left(\eta_{1}\right) \widehat{u}_{2}\left(\xi-\eta_{1}\right)} .
\end{aligned}
$$


Now, observe that for $\left(\xi, \xi_{1}\right)$ fixed, the function $\phi_{1}\left(\eta_{1}\right):=\phi\left(\xi, \xi_{1}, \eta_{1}\right)$ has only two simple roots, $\eta_{1}=\xi_{1}$ and $\eta_{1}=\xi-\xi_{1}$, in the support of $f$. Moreover,

$$
\left|\phi_{1}^{\prime}\left(\eta_{1}\right)\right|=5\left|\left(\eta_{1}^{4}-\left(\xi-\eta_{1}\right)^{4}\right)\right| \geq 5 \quad \text { in } \operatorname{supp} f
$$

and

$$
\left|\phi_{1}^{\prime}\left(\xi_{1}\right)\right|=\left|\phi_{1}^{\prime}\left(\xi-\xi_{1}\right)\right|=5\left|\xi_{1}^{4}-\left(\xi-\xi_{1}\right)^{4}\right| .
$$

Therefore, it follows from the Fourier inversion formula, Fubini's theorem and Plancherel's identity that

$$
\begin{aligned}
\left\|\mathcal{J}^{2}\left(e^{t \partial_{x}^{5}} u_{1}, e^{t \partial_{x}^{5}} u_{2}\right)\right\|_{L_{x, t}^{2}}^{2} & =c \int_{\mathbb{R}^{3}} \delta_{0}\left(\phi\left(\xi, \xi_{1}, \eta_{1}\right)\right) f\left(\xi, \xi_{1} \eta_{1}\right) d \eta_{1} d \xi d \xi_{1} \\
& \lesssim \int_{\mathbb{R}^{2}}\left(\frac{f\left(\xi, \xi_{1}, \xi_{1}\right)}{\left|\phi_{1}^{\prime}\left(\xi_{1}\right)\right|}+\frac{f\left(\xi, \xi_{1}, \xi-\xi_{1}\right)}{\left|\phi_{1}^{\prime}\left(\xi-\xi_{1}\right)\right|}\right) d \xi d \xi_{1} \\
& \lesssim\left\|u_{1}\right\|_{L_{x}^{2}}^{2}\left\|u_{2}\right\|_{L_{x}^{2}}^{2}
\end{aligned}
$$

\section{3. $L^{2}$ BILINEAR AND TRILINEAR ESTIMATES}

3.1. $L^{2}$ bilinear estimates. Recall that $w(\xi)=\xi^{5}$. Then we define the resonance functions $\Omega:=\Omega\left(\xi_{1}, \xi_{2}\right)$ by

$$
\Omega\left(\xi_{1}, \xi_{2}\right):=w\left(\xi_{1}\right)+w\left(\xi_{2}\right)-w\left(\xi_{1}+\xi_{2}\right) .
$$

We first state a technical lemma (see Lemma 3.1 in [8]).

Lemma 3.1. If $\left|\xi_{1}\right| \sim N_{1}, \xi_{2} \sim N_{2}$ and $\left|\xi_{1}+\xi_{2}\right| \sim N$, then

$$
|\Omega| \sim N_{\max }^{4} N_{\min }
$$

Proof. A direct computation shows that

$$
\Omega\left(\xi_{1}, \xi_{2}\right)=-5 \xi_{1} \xi_{2}\left(\xi_{1}+\xi_{2}\right)\left(\xi_{1}^{2}+\xi_{1} \xi_{2}+\xi_{2}^{2}\right),
$$

which implies (3.2), since

$$
\xi_{1}^{2}+\xi_{1} \xi_{2}+\xi_{2}^{2} \sim \max \left\{\xi_{1}^{2}, \xi_{2}^{2}\right\} .
$$

For $k \in \mathbb{Z}_{+}$and $j \in \mathbb{Z}_{+}$, let us define $D_{k, j}$ by

$$
D_{k, j}=\left\{(\xi, \tau): \xi \in I_{k} \text { and }|\tau+w(\xi)| \leq 2^{j}\right\} .
$$

We derive a useful lemma (see also Lemma 2.3 in [7]).

Lemma 3.2. Assume that $k_{1}, k_{2}, k_{3} \in \mathbb{Z}_{+}, j_{1}, j_{2}, j_{3} \in \mathbb{Z}_{+}$and $f_{i}: \mathbb{R}^{2} \rightarrow \mathbb{R}_{+}$are $L^{2}$ functions supported in $D_{k_{i}, j_{i}}$ for $i=1,2,3$.

(a) Then it follows that

$$
\int_{\mathbb{R}^{2}}\left(f_{1} * f_{2}\right) \cdot f_{3} \lesssim 2^{k_{m i n} / 2} 2^{j_{m i n} / 2}\left\|f_{1}\right\|_{L^{2}}\left\|f_{2}\right\|_{L^{2}}\left\|f_{3}\right\|_{L^{2}}
$$

(b) Let us suppose that $k_{\min } \leq k_{\max }-5$. If we are in the case where $\left(k_{i}, j_{i}\right)=$ $\left(k_{\min }, j_{\max }\right)$ for some $i \in\{1,2,3\}$, then it holds that

$$
\int_{\mathbb{R}^{2}}\left(f_{1} * f_{2}\right) \cdot f_{3} \lesssim 2^{\left(j_{1}+j_{2}+j_{3}\right) / 2} 2^{-j_{\operatorname{med}} / 2} 2^{-2 k_{\max }}\left\|f_{1}\right\|_{L^{2}}\left\|f_{2}\right\|_{L^{2}}\left\|f_{3}\right\|_{L^{2}} .
$$




$$
\int_{\mathbb{R}^{2}}\left(f_{1} * f_{2}\right) \cdot f_{3} \lesssim 2^{\left(j_{1}+j_{2}+j_{3}\right) / 2} 2^{-j_{\max } / 2} 2^{-\left(3 k_{\max }+k_{\min }\right) / 2}\left\|f_{1}\right\|_{L^{2}}\left\|f_{2}\right\|_{L^{2}}\left\|f_{3}\right\|_{L^{2}} .
$$

In all the others cases, we have that

$$
\int_{\mathbb{R}^{2}}\left(f_{1} * f_{2}\right) \cdot f_{3} \lesssim 2^{\left(j_{1}+j_{2}+j_{3}\right) / 2} 2^{-j_{\max } / 2} 2^{-2 k_{\max }}\left\|f_{1}\right\|_{L^{2}}\left\|f_{2}\right\|_{L^{2}}\left\|f_{3}\right\|_{L^{2}} .
$$

(c) In the case $\left|k_{\text {min }}-k_{\max }\right| \leq 10, k_{\min } \geq 10$, then we have that

$$
\int_{\mathbb{R}^{2}}\left(f_{1} * f_{2}\right) \cdot f_{3} \lesssim 2^{j_{\min } / 2} 2^{j_{\text {med }} / 4} 2^{-3 k_{\max } / 4}\left\|f_{1}\right\|_{L^{2}}\left\|f_{2}\right\|_{L^{2}}\left\|f_{3}\right\|_{L^{2}}
$$

Proof. First, we begin with the proof of item (a). We observe that

$$
I:=\int_{\mathbb{R}^{2}}\left(f_{1} * f_{2}\right) \cdot f_{3}=\int_{\mathbb{R}^{2}}\left(\widetilde{f}_{1} * f_{3}\right) \cdot f_{2}=\int_{\mathbb{R}^{2}}\left(\widetilde{f}_{2} * f_{3}\right) \cdot f_{1},
$$

where $\tilde{f}_{i}(\xi, \tau)=f_{i}(-\xi,-\tau)$. Therefore, we can always assume that $j_{1}=j_{\text {min }}$. Moreover, let us define $f_{i}^{\sharp}(\xi, \theta)=f_{i}(\xi, \theta+w(\xi))$, for $i=1,2,3$. In view of the assumptions on $f_{i}$, the functions $f_{i}^{\sharp}$ are supported in the sets

$$
D_{k_{i}, j_{i}}^{\sharp}=\left\{(\xi, \theta): \xi \in I_{k_{i}} \text { and }|\theta| \leq 2^{j_{i}}\right\} .
$$

We also note that $\left\|f_{i}\right\|_{L^{2}}=\left\|f_{i}^{\sharp}\right\|_{L^{2}}$. Then, it follows by changing variables that

$$
I=\int_{\mathbb{R}^{4}} f_{1}^{\sharp}\left(\xi_{1}, \theta_{1}\right) f_{2}^{\sharp}\left(\xi_{2}, \theta_{2}\right) f_{3}^{\sharp}\left(\xi_{1}+\xi_{2}, \theta_{1}+\theta_{2}+\Omega\left(\xi_{1}, \xi_{2}\right)\right) d \xi_{1} d \xi_{2} d \theta_{1} d \theta_{2},
$$

where $\Omega\left(\xi_{1}, \xi_{2}\right)$ is defined in (3.1). For $i=1,2,3$, we define $F_{i}(\xi)=\left(\int_{\mathbb{R}} f_{i}^{\sharp}(\xi, \theta)^{2} d \theta\right)^{\frac{1}{2}}$. Thus, it follows by applying the Cauchy-Schwarz and Young inequalities in the $\theta$ variables that

$$
\begin{aligned}
I & \leq \int_{\mathbb{R}^{2}}\left\|f_{1}^{\sharp}\left(\xi_{1}, \cdot\right)\right\|_{L_{\theta}^{1}} F_{2}\left(\xi_{2}\right) F_{3}\left(\xi_{1}+\xi_{2}\right) d \xi_{1} d \xi_{2} \\
& \lesssim 2^{j_{\min } / 2} \int_{\mathbb{R}^{2}} F_{1}\left(\xi_{1}\right) F_{2}\left(\xi_{2}\right) F_{3}\left(\xi_{1}+\xi_{2}\right) d \xi_{1} d \xi_{2} .
\end{aligned}
$$

Estimate (3.6) is deduced from (3.13) by applying the same arguments in the $\xi$ variables.

Next we turn to the proof of item (b). According to (3.11), we can assume that $j_{3}=j_{\max }$. Moreover, it is enough to consider the two cases $k_{\min }=k_{2}$ and $k_{\text {min }}=k_{3}$ (since by symmetry the case $k_{\min }=k_{1}$ is equivalent to the case $k_{\min }=k_{2}$ ).

We prove estimate (3.9) in the case $j_{3}=j_{\max }$ and $k_{\min }=k_{2}$. It suffices to prove that if $g_{i}: \mathbb{R} \rightarrow \mathbb{R}_{+}$are $L^{2}$ functions supported in $I_{k_{i}}$ for $i=1,2$ and $g: \mathbb{R}^{2} \rightarrow \mathbb{R}_{+}$ is an $L^{2}$ function supported in $I_{k_{3}} \times\left[-2^{j_{3}}, 2^{j_{3}}\right]$, then

$$
J\left(g_{1}, g_{2}, g\right):=\int_{\mathbb{R}^{2}} g_{1}\left(\xi_{1}\right) g_{2}\left(\xi_{2}\right) g\left(\xi_{1}+\xi_{2}, \Omega\left(\xi_{1}, \xi_{2}\right)\right) d \xi_{1} d \xi_{2}
$$

satisfies that

$$
J\left(g_{1}, g_{2}, g\right) \lesssim 2^{-2 k_{\max }}\left\|g_{1}\right\|_{L^{2}}\left\|g_{2}\right\|_{L^{2}}\|g\|_{L^{2}}
$$


Indeed, if estimate (3.15) holds, let us define $g_{i}\left(\xi_{i}\right)=f_{i}^{\sharp}\left(\xi_{i}, \theta_{i}\right), i=1,2$, and $g(\xi, \Omega)=f_{3}^{\sharp}\left(\xi, \theta_{1}+\theta_{2}+\Omega\right)$, for $\theta_{1}$ and $\theta_{2}$ fixed. Hence, we would deduce by applying (3.15) and the Cauchy-Schwarz inequality to (3.12) that

$$
\begin{aligned}
I & \lesssim 2^{-2 k_{\max }}\left\|f_{3}^{\sharp}\right\|_{L_{\xi, \theta}^{2}} \int_{\mathbb{R}^{2}}\left\|f_{1}^{\sharp}\left(\cdot, \theta_{1}\right)\right\|_{L_{\xi}^{2}}\left\|f_{2}^{\sharp}\left(\cdot, \theta_{2}\right)\right\|_{L_{\xi}^{2}} d \theta_{1} d \theta_{2} \\
& \lesssim 2^{-2 k_{\max }} 2^{\left(j_{1}+j_{2}\right) / 2}\left\|f_{1}^{\sharp}\right\|_{L_{\xi, \theta}^{2}}\left\|f_{2}^{\sharp}\right\|_{L_{\xi, \theta}^{2}}\left\|f_{3}^{\sharp}\right\|_{L_{\xi, \theta}^{2}},
\end{aligned}
$$

which is estimate (3.9) in this case. To prove estimate (3.15), we apply twice the Cauchy-Schwarz inequality to get that

$$
J\left(g_{1}, g_{2}, g\right) \leq\left\|g_{1}\right\|_{L^{2}}\left\|g_{2}\right\|_{L^{2}}\left(\int_{\mathbb{R}^{2}} g\left(\xi_{1}+\xi_{2}, \Omega\left(\xi_{1}, \xi_{2}\right)\right)^{2} d \xi_{1} d \xi_{2}\right)^{\frac{1}{2}} .
$$

Then we change variables $\left(\xi_{1}^{\prime}, \xi_{2}^{\prime}\right)=\left(\xi_{1}+\xi_{2}, \xi_{2}\right)$ so that

$$
J\left(g_{1}, g_{2}, g\right) \leq\left\|g_{1}\right\|_{L^{2}}\left\|g_{2}\right\|_{L^{2}}\left(\int_{\mathbb{R}^{2}} g\left(\xi_{1}^{\prime}, \Omega\left(\xi_{1}^{\prime}-\xi_{2}^{\prime}, \xi_{2}^{\prime}\right)\right)^{2} d \xi_{1}^{\prime} d \xi_{2}^{\prime}\right)^{\frac{1}{2}} .
$$

We observe that

$$
\left|\frac{\partial}{\partial \xi_{2}^{\prime}} \Omega\left(\xi_{1}^{\prime}-\xi_{2}^{\prime}, \xi_{2}^{\prime}\right)\right|=5\left|\left(\xi_{2}^{\prime}\right)^{4}-\left(\xi_{1}^{\prime}-\xi_{2}^{\prime}\right)^{4}\right| \sim 2^{4 k_{\max }},
$$

since $2^{k_{1}} \sim 2^{k_{\max }}$ by the frequency localization. Then, the change of variables $\mu_{1}=\xi_{1}^{\prime}$ and $\mu_{2}=\Omega\left(\xi_{1}^{\prime}-\xi_{2}^{\prime}, \xi_{2}^{\prime}\right)$ in (3.17) yields (3.15), which concludes the proof of estimate (3.9) in this case.

To prove estimate (3.8) in the case $\left(k_{\min }, j_{\max }\right)=\left(k_{3}, j_{3}\right)$ and $k_{3} \geq 1$, we observe by arguing as above that it suffices to prove that

$$
J\left(g_{1}, g_{2}, g\right) \lesssim 2^{-\left(3 k_{\max }+k_{\min }\right) / 2}\left\|g_{1}\right\|_{L^{2}}\left\|g_{2}\right\|_{L^{2}}\|g\|_{L^{2}},
$$

where $J\left(g_{1}, g_{2}, g\right)$ is defined in (3.14). First, we change variables $\xi_{1}^{\prime}=\xi_{1}$ and $\xi_{2}^{\prime}=\xi_{1}+\xi_{2}$ so that

$$
J\left(g_{1}, g_{2}, g\right)=\int_{\mathbb{R}^{2}} g_{1}\left(\xi_{1}^{\prime}\right) g_{2}\left(\xi_{2}^{\prime}-\xi_{1}^{\prime}\right) g\left(\xi_{2}^{\prime}, \Omega\left(\xi_{1}^{\prime}, \xi_{2}^{\prime}-\xi_{1}^{\prime}\right)\right) d \xi_{1}^{\prime} d \xi_{2}^{\prime} .
$$

The Cauchy-Schwarz inequality implies that

$$
J\left(g_{1}, g_{2}, g\right) \leq\left\|g_{1}\right\|_{L^{2}}\left\|g_{2}\right\|_{L^{2}}\left(\int_{\mathbb{R}^{2}} g\left(\xi_{2}^{\prime}, \Omega\left(\xi_{1}^{\prime}, \xi_{2}^{\prime}-\xi_{1}^{\prime}\right)\right)^{2} d \xi_{1}^{\prime} d \xi_{2}^{\prime}\right)^{\frac{1}{2}}
$$

We compute that

$$
\left|\frac{\partial}{\partial \xi_{1}^{\prime}} \Omega\left(\xi_{1}^{\prime}, \xi_{2}^{\prime}-\xi_{1}^{\prime}\right)\right|=5\left|\left(\xi_{1}^{\prime}\right)^{4}-\left(\xi_{2}^{\prime}-\xi_{1}^{\prime}\right)^{4}\right| \sim 2^{3 k_{\max }+k_{\min }},
$$

since $\left|\xi_{1}^{\prime}\right| \sim 2^{k_{\max }}$ and $\left|\xi_{2}^{\prime}\right| \sim 2^{k_{\min }}$ due to the frequency localization. Therefore estimate (3.18) is deduced by performing the change of variables $\mu_{1}^{\prime}=\Omega\left(\xi_{1}^{\prime}, \xi_{2}^{\prime}-\xi_{1}^{\prime}\right)$ and $\mu_{2}^{\prime}=\xi_{2}^{\prime}$ in (3.19). On the other hand, by writing

$$
I=\int_{\mathbb{R}^{2}}\left(\tilde{f}_{1} * f_{3}\right) \cdot f_{2}
$$

and arguing as in (3.16), we get estimate (3.7) in the case $\left(k_{\min }, j_{\max }\right)=\left(k_{3}, j_{3}\right)$. 
Estimate (3.10) is stated in Lemma 2.3 (c) of [7, and its proof follows closely the one for the dispersion generalized BO in [11. However, for the sake of completeness we will derive it here. According to (3.11), we may assume that $j_{\max }=j_{3}$. Furthermore, we have following (3.12) that

$$
\begin{aligned}
I & =\sum_{i=1}^{3} \int_{\mathcal{R}_{i}} f_{1}^{\sharp}\left(\xi_{1}, \theta_{1}\right) f_{2}^{\sharp}\left(\xi_{2}, \theta_{2}\right) f_{3}^{\sharp}\left(\xi_{1}+\xi_{2}, \theta_{1}+\theta_{2}+\Omega\left(\xi_{1}, \xi_{2}\right)\right) d \xi_{1} d \xi_{2} d \theta_{1} d \theta_{2} \\
& =: I_{1}+I_{2}+I_{3},
\end{aligned}
$$

where

$$
\begin{aligned}
& \mathcal{R}_{1}=\left\{\left(\xi_{1}, \xi_{2}, \theta_{1}, \theta_{2}\right) \in \mathbb{R}^{4}: \xi_{1} \cdot \xi_{2}<0\right\}, \\
& \mathcal{R}_{2}=\left\{\left(\xi_{1}, \xi_{2}, \theta_{1}, \theta_{2}\right) \in \mathbb{R}^{4}: \xi_{1} \cdot \xi_{2}>0 \text { and }\left|\xi_{1}-\xi_{2}\right|<R\right\}, \\
& \mathcal{R}_{3}=\left\{\left(\xi_{1}, \xi_{2}, \theta_{1}, \theta_{2}\right) \in \mathbb{R}^{4}: \xi_{1} \cdot \xi_{2}>0 \text { and }\left|\xi_{1}-\xi_{2}\right|>R\right\},
\end{aligned}
$$

and $R$ is a positive number which will be chosen later.

First we prove that

$$
I_{1} \lesssim 2^{\left(j_{\text {min }}+j_{\text {med }}\right) / 2} 2^{-2 k_{\max }}\left\|f_{1}\right\|_{L^{2}}\left\|f_{2}\right\|_{L^{2}}\left\|f_{3}\right\|_{L^{2}},
$$

which would imply that

$$
I_{1} \lesssim 2^{j_{\text {min }} / 2} 2^{j_{\text {med }} / 4} 2^{-k_{\max }}\left\|f_{1}\right\|_{L^{2}}\left\|f_{2}\right\|_{L^{2}}\left\|f_{3}\right\|_{L^{2}},
$$

after interpolating with estimate (3.6).

To prove (3.21), we argue as for (3.9), so that it suffices to prove

$$
J\left(g_{1}, g_{2}, g\right) \lesssim 2^{-2 k_{\max }}\left\|g_{1}\right\|_{L^{2}}\left\|g_{2}\right\|_{L^{2}}\|g\|_{L^{2}},
$$

where $J\left(g_{1}, g_{2}, g\right)$ is defined as in (3.14). By symmetry, we can always assume that $\left|\xi_{1}\right| \leq\left|\xi_{2}\right|$. We apply twice the Cauchy-Schwarz inequality and perform the change of variables $\left(\xi_{1}^{\prime}, \xi_{2}^{\prime}\right)=\left(\xi_{1}, \xi_{1}+\xi_{2}\right)$ to obtain that

$$
J\left(g_{1}, g_{2}, g\right) \leq\left\|g_{1}\right\|_{L^{2}}\left\|g_{2}\right\|_{L^{2}}\left(\int_{\mathbb{R}^{2}} g\left(\xi_{2}^{\prime}, \Omega\left(\xi_{1}^{\prime}, \xi_{2}^{\prime}-\xi_{1}^{\prime}\right)\right)^{2} d \xi_{1}^{\prime} d \xi_{2}^{\prime}\right)^{\frac{1}{2}} .
$$

Now observe that

$$
\left|\frac{\partial}{\partial \xi_{1}^{\prime}} \Omega\left(\xi_{1}^{\prime}, \xi_{2}^{\prime}-\xi_{1}^{\prime}\right)\right|=5\left|\left(\xi_{2}^{\prime}\right)^{4}-4\left(\xi_{2}^{\prime}\right)^{3} \xi_{1}^{\prime}+6\left(\xi_{2}^{\prime}\right)^{2}\left(\xi_{1}^{\prime}\right)^{2}-4 \xi_{2}^{\prime}\left(\xi_{1}^{\prime}\right)^{3}\right| \sim 2^{4 k_{\max }},
$$

due to the frequency localization and the restriction $\xi_{1}^{\prime} \cdot \xi_{2}^{\prime} \leq 0$ (which is a consequence of the assumptions $\xi_{1} \cdot \xi_{2}<0$ and $\left.\left|\xi_{1}\right| \leq\left|\xi_{2}\right|\right)$. Therefore, the change of variables $\left(\mu_{1}^{\prime}, \mu_{2}^{\prime}\right)=\left(\Omega\left(\xi_{1}^{\prime}, \xi_{2}^{\prime}-\xi_{1}^{\prime}\right), \xi_{2}^{\prime}\right)$ in (3.24) yields estimate (3.23).

To deal with $I_{2}$, we get as in (3.13) that

$$
I_{2} \lesssim 2^{j_{\min } / 2} \int_{\left|\xi_{1}-\xi_{2}\right|<R} F_{1}\left(\xi_{1}\right) F_{2}\left(\xi_{2}\right) F_{3}\left(\xi_{1}+\xi_{2}\right) d \xi_{1} d \xi_{2}
$$

Then, we obtain by letting $\left(\xi_{1}^{\prime}, \xi_{2}^{\prime}\right)=\left(\xi_{1}-\xi_{2}, \xi_{2}\right)$ and applying twice the CauchySchwarz inequality that

$$
\begin{aligned}
I_{2} & \lesssim 2^{j_{\min } / 2} \int_{\left|\xi_{1}^{\prime}\right|<R} F_{1}\left(\xi_{1}^{\prime}+\xi_{2}^{\prime}\right) F_{2}\left(\xi_{2}^{\prime}\right) F_{3}\left(\xi_{1}^{\prime}+2 \xi_{2}^{\prime}\right) d \xi_{1}^{\prime} d \xi_{2}^{\prime} \\
& \lesssim 2^{j_{\min } / 2} R^{1 / 2}\left\|f_{1}\right\|_{L^{2}}\left\|f_{2}\right\|_{L^{2}}\left\|f_{3}\right\|_{L^{2}} .
\end{aligned}
$$


Next, we observe that in the region $\mathcal{R}_{3}$,

$$
\left|\xi_{1}^{4}-\xi_{2}^{4}\right|^{\frac{1}{2}}=\left(\left|\xi_{1}-\xi_{2}\right| \cdot\left|\xi_{1}^{3}+\xi_{1}^{2} \xi_{2}+\xi_{1} \xi_{2}^{2}+\xi_{2}^{3}\right|\right)^{\frac{1}{2}} \geq c R^{1 / 2} 2^{3 k_{\max } / 2} \geq 2,
$$

since $R$ will be chosen large enough. Thus, the Cauchy-Schwarz inequality implies that

$$
\begin{aligned}
I_{3} & \lesssim R^{-1 / 2} 2^{-3 k_{\max } / 2}\left\|f_{3}\right\|_{L^{2}} \\
& \times\left\|\int_{\substack{\xi_{1}+\xi_{2}=\xi \\
\theta_{1}+\theta_{2}=\theta}}\left|\xi_{1}^{4}-\xi_{2}^{4}\right|_{1}^{\frac{1}{2}} f_{1}\left(\xi_{1}, \theta_{1}+w\left(\xi_{1}\right)\right) f_{2}\left(\xi_{2}, \theta_{2}+w\left(\xi_{2}\right)\right) d \xi_{1} d \theta_{1}\right\|_{L_{\xi, \theta}^{2}},
\end{aligned}
$$

where the definition of $|\cdot|_{1}$ is given just before Lemma2.15. By Plancherel's identity, the $L^{2}$-norm of the integral on the right-hand side of (3.26) is equal to

$\left\|\int_{\theta_{1}, \theta_{2}} e^{-i t\left(\theta_{1}+\theta_{2}\right)} \int_{\xi_{1}+\xi_{2}=\xi}\left|\xi_{1}^{4}-\xi_{2}^{4}\right|_{1}^{\frac{1}{2}} f_{1}\left(\xi_{1}, \theta_{1}+w\left(\xi_{1}\right)\right) f_{2}\left(\xi_{2}, \theta_{2}+w\left(\xi_{2}\right)\right) d \xi_{1} d \theta_{1} d \theta_{2}\right\|_{L_{\xi, t}^{2}}$.

This implies after changing variables $\tau_{i}=\theta_{i}+w\left(\xi_{i}\right)$ for $i=1,2$ and using Minkowski's inequality that

$$
\begin{aligned}
& I_{3} \lesssim R^{-1 / 2} 2^{-3 k_{\max } / 2}\left\|f_{3}\right\|_{L^{2}}
\end{aligned}
$$

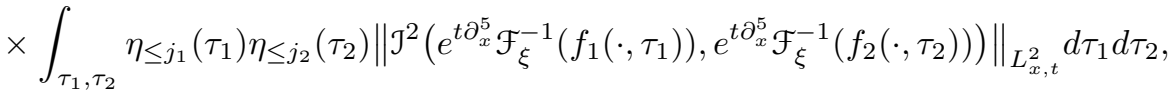

where the bilinear operator $\mathcal{J}^{2}$ is defined in Lemma 2.15. Therefore, we deduce from estimate (2.53) and the Cauchy-Schwarz inequality that

$$
I_{3} \lesssim R^{-1 / 2} 2^{-3 k_{\max } / 2} 2^{j_{\text {min }} / 2} 2^{j_{\text {med }} / 2}\left\|f_{1}\right\|_{L^{2}}\left\|f_{2}\right\|_{L^{2}}\left\|f_{3}\right\|_{L^{2}} .
$$

Finally, we conclude estimate (3.10) by gathering estimates (3.20), (3.22), (3.25), (3.27) and choosing $R=2^{-3 k_{\max } / 2} 2^{j_{\text {med }} / 2}$.

This finishes the proof of Lemma 3.2 .

As a consequence of Lemma 3.2 , we have the following $L^{2}$ bilinear estimates.

Corollary 3.3. Assume that $k_{1}, k_{2}, k_{3} \in \mathbb{Z}_{+}, j_{1}, j_{2}, j_{3} \in \mathbb{Z}_{+}$and $f_{i}: \mathbb{R}^{2} \rightarrow \mathbb{R}_{+}$ are $L^{2}$ functions supported in $D_{k_{i}, j_{i}}$ for $i=1,2$.

(a) Then it follows that

$$
\left\|\mathbf{1}_{D_{k_{3}, j_{3}}} \cdot\left(f_{1} * f_{2}\right)\right\|_{L^{2}} \lesssim 2^{k_{m i n} / 2} 2^{j_{m i n} / 2}\left\|f_{1}\right\|_{L^{2}}\left\|f_{2}\right\|_{L^{2}} .
$$

(b) Let us suppose that $k_{\min } \leq k_{\max }-5$. If we are in the case where $\left(k_{i}, j_{i}\right)=$ $\left(k_{\min }, j_{\max }\right)$ for some $i \in\{1,2,3\}$, then it holds that

$$
\left\|\mathbf{1}_{D_{k_{3}, j_{3}}} \cdot\left(f_{1} * f_{2}\right)\right\|_{L^{2}} \lesssim 2^{\left(j_{1}+j_{2}+j_{3}\right) / 2} 2^{-j_{\text {med }} / 2} 2^{-2 k_{\max }}\left\|f_{1}\right\|_{L^{2}}\left\|f_{2}\right\|_{L^{2}} .
$$

If moreover $k_{\text {min }} \geq 1$, then

$$
\left\|\mathbf{1}_{D_{k_{3}, j_{3}}} \cdot\left(f_{1} * f_{2}\right)\right\|_{L^{2}} \lesssim 2^{\left(j_{1}+j_{2}+j_{3}\right) / 2} 2^{-j_{\max } / 2} 2^{-\left(3 k_{\max }+k_{\min }\right) / 2}\left\|f_{1}\right\|_{L^{2}}\left\|f_{2}\right\|_{L^{2}} .
$$

In all the others cases, we have that

$$
\left\|\mathbf{1}_{D_{k_{3}, j_{3}}} \cdot\left(f_{1} * f_{2}\right)\right\|_{L^{2}} \lesssim 2^{\left(j_{1}+j_{2}+j_{3}\right) / 2} 2^{-j_{\max } / 2} 2^{-2 k_{\max }}\left\|f_{1}\right\|_{L^{2}}\left\|f_{2}\right\|_{L^{2}} .
$$

Proof. The proof of Corollary 3.3 follows directly from Lemma 3.2 by using a duality argument. 
3.2. $L^{2}$ trilinear estimates. Now, we prove the $L^{2}$ trilinear estimates. In this case, the resonance function $\widetilde{\Omega}:=\widetilde{\Omega}\left(\xi_{1}, \xi_{2}, \xi_{3}\right)$ is given by

$$
\widetilde{\Omega}\left(\xi_{1}, \xi_{2}, \xi_{3}\right):=w\left(\xi_{1}\right)+w\left(\xi_{2}\right)+w\left(\xi_{3}\right)-w\left(\xi_{1}+\xi_{2}+\xi_{3}\right) .
$$

Lemma 3.4. Assume that $k_{1}, k_{2}, k_{3}, k_{4} \in \mathbb{Z}_{+}, j_{1}, j_{2}, j_{3}, j_{4} \in \mathbb{Z}_{+}$, and $f_{i}$ : $\mathbb{R}^{2} \rightarrow \mathbb{R}_{+}$are $L^{2}$ functions supported in $D_{k_{i}, j_{i}}$ for $i=1,2,3,4$.

(a) Then it follows that

$$
\int_{\mathbb{R}^{2}}\left(f_{1} * f_{2} * f_{3}\right) \cdot f_{4} \lesssim 2^{\left(k_{\text {min }}+k_{t h d}\right) / 2} 2^{\left(j_{\min }+j_{\text {thd }}\right) / 2}\left\|f_{1}\right\|_{L^{2}}\left\|f_{2}\right\|_{L^{2}}\left\|f_{3}\right\|_{L^{2}}\left\|f_{4}\right\|_{L^{2}}
$$

(b) Moreover, let us suppose that $k_{\text {thd }} \leq k_{\max }-5$. If we are in the case where $\left(k_{i}, j_{i}\right)=\left(k_{\text {thd }}, j_{\max }\right)$ for some $i \in\{1,2,3,4\}$, then it holds that

$$
\begin{aligned}
& \int_{\mathbb{R}^{2}}\left(f_{1} * f_{2} * f_{3}\right) \cdot f_{4} \\
& \quad \lesssim 2^{\left(j_{1}+j_{2}+j_{3}+j_{4}\right) / 2} 2^{-j_{\max } / 2} 2^{k_{t h d} / 2} 2^{-2 k_{\max }}\left\|f_{1}\right\|_{L^{2}}\left\|f_{2}\right\|_{L^{2}}\left\|f_{3}\right\|_{L^{2}}\left\|f_{4}\right\|_{L^{2}} \\
& \text { and } \\
& \int_{\mathbb{R}^{2}}\left(f_{1} * f_{2} * f_{3}\right) \cdot f_{4} \\
& \quad \lesssim 2^{\left(j_{1}+j_{2}+j_{3}+j_{4}\right) / 2} 2^{-j_{\operatorname{med}} / 2} 2^{k_{\min } / 2} 2^{-2 k_{\max }}\left\|f_{1}\right\|_{L^{2}}\left\|f_{2}\right\|_{L^{2}}\left\|f_{3}\right\|_{L^{2}}\left\|f_{4}\right\|_{L^{2}} .
\end{aligned}
$$

In all the others cases, we have that

$$
\begin{aligned}
& \int_{\mathbb{R}^{2}}\left(f_{1} * f_{2} * f_{3}\right) \cdot f_{4} \\
& \quad \lesssim 2^{\left(j_{1}+j_{2}+j_{3}+j_{4}\right) / 2} 2^{-j_{\max } / 2} 2^{k_{\min } / 2} 2^{-2 k_{\max }}\left\|f_{1}\right\|_{L^{2}}\left\|f_{2}\right\|_{L^{2}}\left\|f_{3}\right\|_{L^{2}}\left\|f_{4}\right\|_{L^{2}} .
\end{aligned}
$$

Proof. Estimate (3.33) can be proved exactly as estimate (3.6). To prove part (b), we follow closely the arguments of Guo for the mBO equation [12. Let us define

$$
\widetilde{I}:=\int_{\mathbb{R}^{2}}\left(f_{1} * f_{2} * f_{3}\right) \cdot f_{4} .
$$

Observe that

$$
\widetilde{I}=\int_{\mathbb{R}^{2}}\left(\tilde{f}_{1} * \widetilde{f}_{2} * f_{4}\right) \cdot f_{3}=\int_{\mathbb{R}^{2}}\left(\widetilde{f}_{2} * \widetilde{f}_{2} * f_{4}\right) \cdot f_{1}=\int_{\mathbb{R}^{2}}\left(\widetilde{f}_{1} * \widetilde{f}_{3} * f_{4}\right) \cdot f_{2},
$$

where $\tilde{f}_{i}(\xi, \tau)=f_{i}(-\xi,-\tau)$. Therefore, we can always assume that $j_{\max }=j_{4}$. Moreover, we introduce $f_{i}^{\sharp}(\xi, \theta)=f_{i}(\xi, \theta+w(\xi))$, for $i=1,2,3$. In view of the assumptions on $f_{i}$, the functions $f_{i}^{\sharp}$ are supported in the sets

$$
D_{k_{i}, j_{i}}^{\sharp}=\left\{(\xi, \theta): \xi \in I_{k_{i}} \text { and }|\theta| \leq 2^{j_{i}}\right\} .
$$

We also note that $\left\|f_{i}\right\|_{L^{2}}=\left\|f_{i}^{\sharp}\right\|_{L^{2}}$. Then, it follows by changing variables that (3.39)

$$
\widetilde{I}=\int_{\mathbb{R}^{6}} f_{1}^{\sharp}\left(\xi_{1}, \theta_{1}\right) f_{2}^{\sharp}\left(\xi_{2}, \theta_{2}\right) f_{3}^{\sharp}\left(\xi_{3}, \theta_{3}\right) f_{4}^{\sharp}\left(\xi_{1}+\xi_{2}+\xi_{3}, \theta_{1}+\theta_{2}+\theta_{3}+\widetilde{\Omega}\left(\xi_{1}, \xi_{2}, \xi_{3}\right)\right) d \nu,
$$

where $d \nu=d \xi_{1} d \xi_{2} d \xi_{3} d \theta_{1} d \theta_{2} d \theta_{3}$ and $\tilde{\Omega}\left(\xi_{1}, \xi_{2}\right)$ is defined in (3.32).

Since $k_{t h d} \leq k_{\max }-5$ by hypothesis, we always have that $k_{\max } \sim k_{s u b}$. Thus, we only need to treat the following cases: $k_{4} \sim k_{\max }, k_{4}=k_{t h d}$ and $k_{4}=k_{\min }$. 
Case $k_{4} \sim k_{\max }$. By symmetry, we can assume that $k_{1} \leq k_{2} \leq k_{3} \leq k_{4}$ in this case. For $g_{i}: \mathbb{R} \rightarrow \mathbb{R}_{+}, L^{2}$ functions supported in $I_{k_{i}}$ for $i=1,2,3$ and $g: \mathbb{R}^{2} \rightarrow \mathbb{R}_{+}$, an $L^{2}$ function supported in $I_{k_{4}} \times\left[-2^{j_{4}}, 2^{j_{4}}\right]$, let us define

$$
\widetilde{J}\left(g_{1}, g_{2}, g_{3}, g\right):=\int_{\mathbb{R}^{3}} g_{1}\left(\xi_{1}\right) g_{2}\left(\xi_{2}\right) g_{3}\left(\xi_{3}\right) g\left(\xi_{1}+\xi_{2}+\xi_{3}, \widetilde{\Omega}\left(\xi_{1}, \xi_{2}, \xi_{3}\right)\right) d \xi_{1} d \xi_{2} d \xi_{3}
$$

Then, arguing as in (3.16), it suffices to show that

$$
\widetilde{J}\left(g_{1}, g_{2}, g_{3}, g\right) \lesssim 2^{-2 k_{\max }} 2^{k_{\min } / 2}\left\|g_{1}\right\|_{L^{2}}\left\|g_{2}\right\|_{L^{2}}\left\|g_{3}\right\|_{L^{2}}\|g\|_{L^{2}}
$$

in order to prove (3.36) in this case. To prove estimate (3.41), we change variables $\left(\xi_{1}^{\prime}, \xi_{2}^{\prime}, \xi_{3}^{\prime}\right)=\left(\xi_{1}, \xi_{2}, \xi_{1}+\xi_{2}+\xi_{3}\right)$ and apply twice the Cauchy-Schwarz inequality in the $\xi_{1}^{\prime}$ and $\xi_{2}^{\prime}$ to deduce that

$$
\widetilde{J}\left(g_{1}, g_{2}, g_{3}, g\right) \lesssim\left\|g_{2}\right\|_{L^{2}}\left\|g_{3}\right\|_{L^{2}} \int_{\left|\xi_{1}^{\prime}\right| \sim 2^{k_{1}}} g_{1}\left(\xi_{1}^{\prime}\right)\left(\int_{\mathbb{R}^{2}} g\left(\xi_{3}^{\prime}, \widetilde{\Omega}\right)^{2} d \xi_{2}^{\prime} d \xi_{3}\right)^{\frac{1}{2}} d \xi_{1} .
$$

We observe that

$$
\left|\frac{\partial}{\partial \xi_{2}^{\prime}} \widetilde{\Omega}\left(\xi_{1}^{\prime}, \xi_{2}^{\prime}, \xi_{3}^{\prime}-\xi_{2}^{\prime}-\xi_{1}^{\prime}\right)\right|=5\left|\left(\xi_{2}^{\prime}\right)^{4}-\left(\xi_{3}^{\prime}-\xi_{2}^{\prime}+\xi_{1}^{\prime}\right)^{4}\right| \sim 2^{4 k_{\max }}
$$

by using the frequency localization. Thus estimate (3.41) is deduced by performing the change of variables $\left(\mu_{2}, \mu_{3}\right)=\left(\widetilde{\Omega}, \xi_{3}^{\prime}\right)$ in the inner integral on the right-hand side of (3.42) and by applying the Cauchy-Schwarz inequality in the variable $\xi_{1}^{\prime}$.

Case $k_{4} \sim k_{\min }$. In this case, we can assume without loss of generality that $k_{4} \leq$ $k_{1} \leq k_{2} \leq k_{3}$. It suffices to show that estimate (3.41) remains valid in this case. First, we change variables $\left(\xi_{1}^{\prime}, \xi_{2}^{\prime}, \xi_{3}^{\prime}\right)=\left(\xi_{1}, \xi_{2}, \xi_{1}+\xi_{2}+\xi_{3}\right)$ so that $\left|\xi_{1}^{\prime}\right| \sim 2^{k_{\text {thd }}}$, $\left|\xi_{2}^{\prime}\right| \sim 2^{k_{\max }},\left|\xi_{3}^{\prime}\right| \sim 2^{k_{\min }}$ and $\widetilde{J}$ becomes

$$
\begin{aligned}
& \widetilde{J}\left(g_{1}, g_{2}, g_{3}, g\right) \\
& \quad=\int_{\mathbb{R}^{3}} g_{1}\left(\xi_{1}^{\prime}\right) g_{2}\left(\xi_{2}^{\prime}\right) g_{3}\left(\xi_{3}^{\prime}-\xi_{1}^{\prime}-\xi_{2}^{\prime}\right) g\left(\xi_{3}^{\prime}, \widetilde{\Omega}\left(\xi_{1}^{\prime}, \xi_{2}^{\prime}, \xi_{3}^{\prime}-\xi_{1}^{\prime}-\xi_{2}^{\prime}\right)\right) d \xi_{1}^{\prime} d \xi_{2}^{\prime} d \xi_{3}^{\prime} .
\end{aligned}
$$

Thus the Cauchy-Schwarz inequality in $\xi_{1}^{\prime}$ implies that

$$
\begin{aligned}
& \widetilde{J}\left(g_{1}, g_{2}, g_{3}, g\right) \\
& \leq \int_{\mathbb{R}^{2}} g_{2}\left(\xi_{2}^{\prime}\right)\left\|g_{1}\left(\xi_{1}^{\prime}\right) g_{3}\left(\xi_{3}^{\prime}-\xi_{2}^{\prime}-\xi_{1}^{\prime}\right)\right\|_{L_{\xi_{1}^{\prime}}^{2}}\left\|g\left(\xi_{3}^{\prime}, \widetilde{\Omega}\left(\xi_{1}^{\prime}, \xi_{2}^{\prime}, \xi_{3}^{\prime}-\xi_{1}^{\prime}-\xi_{2}^{\prime}\right)\right)\right\|_{L_{\xi_{1}^{\prime}}^{2}} d \xi_{2}^{\prime} d \xi_{3}^{\prime} .
\end{aligned}
$$

Moreover, we have that

$$
\left|\frac{\partial}{\partial \xi_{1}^{\prime}} \widetilde{\Omega}\left(\xi_{1}^{\prime}, \xi_{2}^{\prime}, \xi_{3}^{\prime}-\xi_{2}^{\prime}-\xi_{1}^{\prime}\right)\right|=5\left|\left(\xi_{1}^{\prime}\right)^{4}-\left(\xi_{3}^{\prime}-\xi_{1}^{\prime}-\xi_{2}^{\prime}\right)^{4}\right| \sim 2^{4 k_{\max }},
$$

due to the frequency localization, so that we deduce through the change of variable $\mu_{1}^{\prime}=\widetilde{\Omega}$ that

$$
\left\|g\left(\xi_{3}^{\prime}, \widetilde{\Omega}\left(\xi_{1}^{\prime}, \xi_{2}^{\prime}, \xi_{3}^{\prime}-\xi_{1}^{\prime}-\xi_{2}^{\prime}\right)\right)\right\|_{L_{\xi_{1}^{\prime}}^{2}}=c 2^{-2 k_{\max }}\left\|g\left(\xi_{3}^{\prime}, \cdot\right)\right\|_{L^{2}}
$$


Therefore, we deduce by inserting (3.44) into (3.43) and applying twice the CauchySchwarz inequality that

$$
\begin{aligned}
\widetilde{J}\left(g_{1}, g_{2}, g_{3}, g\right) & \lesssim 2^{-2 k_{\max }}\left\|g_{1}\right\|_{L^{2}}\left\|g_{2}\right\|_{L^{2}}\left\|g_{3}\right\|_{L^{2}} \int_{\left|\xi_{3}^{\prime}\right| \sim 2^{k_{m i n}}}\left\|g\left(\xi_{3}^{\prime}, \cdot\right)\right\|_{L^{2}} d \xi_{3}^{\prime} \\
& \lesssim 2^{-2 k_{\max }} 2^{k_{\min } / 2}\left\|g_{1}\right\|_{L^{2}}\left\|g_{2}\right\|_{L^{2}}\left\|g_{3}\right\|_{L^{2}}\|g\|_{L^{2}}
\end{aligned}
$$

which is exactly (3.41).

Case $k_{4}=k_{t h d}$. Estimate (3.34) follows, arguing exactly as in the case $k_{4}=k_{m i n}$. On the other hand, estimate (3.35) can also be proved by applying the arguments of the cases $k_{4} \sim k_{\max }$ or $k_{4}=k_{\text {min }}$, depending on whether $j_{m e d}=j_{1}, j_{2}$ or $j_{3}$ and using the symmetry relation (3.38).

As a consequence of Lemma 3.4, we have the following $L^{2}$ trilinear estimates.

Corollary 3.5. Assume that $k_{1}, k_{2}, k_{3}, k_{4} \in \mathbb{Z}_{+}, j_{1}, j_{2}, j_{3}, j_{4} \in \mathbb{Z}_{+}$and $f_{i}: \mathbb{R}^{2} \rightarrow \mathbb{R}_{+}$are $L^{2}$ functions supported in $D_{k_{i}, j_{i}}$ for $i=1,2,3$.

(a) Then it follows that

(b) Let us suppose that $k_{\text {thd }} \leq k_{\max }-5$. If we are in the case where $\left(k_{i}, j_{i}\right)=$ $\left(k_{\text {thd }}, j_{\max }\right)$ for some $i \in\{1,2,3,4\}$, then it holds that

$$
\begin{aligned}
\| \mathbf{1}_{D_{k_{4}, j_{4}}} & \cdot\left(f_{1} * f_{2} * f_{3}\right) \|_{L^{2}} \\
& \lesssim 2^{\left(j_{1}+j_{2}+j_{3}+j_{4}\right) / 2} 2^{-j_{\max } / 2} 2^{k_{t h d} / 2} 2^{-2 k_{\max }}\left\|f_{1}\right\|_{L^{2}}\left\|f_{2}\right\|_{L^{2}}\left\|f_{3}\right\|_{L^{2}}
\end{aligned}
$$

and

$$
\begin{aligned}
\| \mathbf{1}_{D_{k_{4}, j_{4}}} & \cdot\left(f_{1} * f_{2} * f_{3}\right) \|_{L^{2}} \\
& \lesssim 2^{\left(j_{1}+j_{2}+j_{3}+j_{4}\right) / 2} 2^{-j_{\text {med }} / 2} 2^{k_{\text {min }} / 2} 2^{-2 k_{\text {max }}}\left\|f_{1}\right\|_{L^{2}}\left\|f_{2}\right\|_{L^{2}}\left\|f_{3}\right\|_{L^{2}} .
\end{aligned}
$$

In all the others cases, we have that

$$
\begin{aligned}
\| \mathbf{1}_{D_{k_{4}, j_{4}}} & \cdot\left(f_{1} * f_{2} * f_{3}\right) \|_{L^{2}} \\
& \lesssim 2^{\left(j_{1}+j_{2}+j_{3}+j_{4}\right) / 2} 2^{-j_{\max } / 2} 2^{k_{\min } / 2} 2^{-2 k_{\max }}\left\|f_{1}\right\|_{L^{2}}\left\|f_{2}\right\|_{L^{2}}\left\|f_{3}\right\|_{L^{2}} .
\end{aligned}
$$

Proof. Corollary 3.5 follows directly from Lemma 3.4 by using a duality argument.

\section{Short time Bilinear estimates}

The main results of this section are the following bilinear estimates in the $F_{\alpha}^{s}(T)$ spaces. Note that to overcome the high-low frequency interaction problem (cf. [32]), we need to work with $\alpha=2$ (see Lemma 4.3 below). Therefore, we will fix $\alpha=2$ in the rest of the paper and denote respectively $F_{2}^{s}(T), N_{2}^{s}(T), F_{2}^{s}, N_{2}^{s}, F_{k, 2}$ and $N_{k, 2}$ by $F^{s}(T), N^{s}(T), F^{s}, N^{s}, F_{k}$ and $N_{k}$. The main results of this section are the bilinear estimates at the $H^{s}$ and $L^{2}$ level.

Proposition 4.1. Let $s>1$ and $T \in(0,1]$ be given. Then, it holds that

$$
\left\|\partial_{x}\left(u \partial_{x}^{2} v\right)\right\|_{N^{s}(T)} \lesssim\|u\|_{F^{s}(T)}\|v\|_{F^{1}(T)}+\|u\|_{F^{1}(T)}\|v\|_{F^{s}(T)}
$$

and

$$
\left\|\partial_{x} u \partial_{x}^{2} v\right\|_{N^{s}(T)} \lesssim\|u\|_{F^{s}(T)}\|v\|_{F^{1}(T)}+\|u\|_{F^{1}(T)}\|v\|_{F^{s}(T)},
$$

for all $u, v \in F^{s}(T)$. 
Proposition 4.2. Let $T \in(0,1]$ be given. Then, it holds that

$$
\left\|\partial_{x}\left(u \partial_{x}^{2} v\right)\right\|_{N^{0}(T)}+\left\|\partial_{x}\left(v \partial_{x}^{2} u\right)\right\|_{N^{0}(T)} \lesssim\|u\|_{F^{2}(T)}\|v\|_{F^{0}(T)}
$$

and

$$
\left\|\partial_{x}\left(\partial_{x} u \partial_{x} v\right)\right\|_{N^{0}(T)} \lesssim\|u\|_{F^{2}(T)}\|v\|_{F^{0}(T)},
$$

for all $u \in F^{2}(T)$ and $v \in F^{0}(T)$.

We split the proof of Propositions 4.1 and 4.2 into several technical lemmas.

Lemma 4.3 (high $\times$ low $\rightarrow$ high). Assume that $k, k_{1}, k_{2} \in \mathbb{Z}_{+}$satisfy $\left|k-k_{2}\right| \leq 3$ and $0 \leq k_{1} \leq \max \left(k, k_{2}\right)-5$. Then,

$$
\left\|P_{k} \partial_{x}\left(u_{k_{1}} \partial_{x}^{2} v_{k_{2}}\right)\right\|_{N_{k}} \lesssim\left\|u_{k_{1}}\right\|_{F_{k_{1}}}\left\|v_{k_{2}}\right\|_{F_{k_{2}}}
$$

and

$$
\left\|P_{k}\left(\partial_{x} u_{k_{1}} \partial_{x}^{2} v_{k_{2}}\right)\right\|_{N_{k}} \lesssim\left\|u_{k_{1}}\right\|_{F_{k_{1}}}\left\|v_{k_{2}}\right\|_{F_{k_{2}}},
$$

for all $u_{k_{1}} \in F_{k_{1}}$ and $v_{k_{2}} \in F_{k_{2}}$.

Remark 4.4. In the case $k_{1}=0$, the function $u_{0} \in F_{0}$ is localized in spatial low frequencies corresponding to the projection $P_{\leq 0}$, since we choose to use a nonhomogeneous dyadic partition of the unity to define the function spaces $F^{s}$ and $N^{s}$ (see Section 2).

Remark 4.5. Lemma 4.3 still holds true under the assumptions $k, k_{1}, k_{2} \in \mathbb{Z}_{+}$, $\left|k-k_{1}\right| \leq 3$ and $0 \leq k_{2} \leq \max \left(k, k_{1}\right)-5$. The proof is exactly the same; therefore we will omit it.

Proof of Lemma 4.3. We prove only estimate (4.5), since the proof of estimate (4.6) is similar (and even easier). First, observe from the definition of $N_{k}$ in (2.6) that

$$
\left\|P_{k} \partial_{x}\left(u_{k_{1}} \partial_{x}^{2} v_{k_{2}}\right)\right\|_{N_{k}} \lesssim \sup _{t_{k} \in \mathbb{R}}\left\|\left(\tau-w(\xi)+i 2^{2 k}\right)^{-1} 2^{3 k} \mathbf{1}_{I_{k}} f_{k_{1}} * f_{k_{2}}\right\|_{X_{k}},
$$

where

$$
f_{k_{1}}=\left|\mathcal{F}\left(\eta_{0}\left(2^{2 k}\left(\cdot-t_{k}\right)\right) u_{k_{1}}\right)\right| \quad \text { and } \quad f_{k_{2}}=\left|\mathcal{F}\left(\widetilde{\eta}_{0}\left(2^{2 k}\left(\cdot-t_{k}\right)\right) v_{k_{2}}\right)\right| .
$$

Now, we set

$$
f_{k_{i}, 2 k}(\xi, \tau)=\eta_{\leq 2 k}(\tau-w(\xi)) f_{k_{i}}(\xi, \tau) \text { and } f_{k_{i}, j_{i}}(\xi, \tau)=\eta_{j_{i}}(\tau-w(\xi)) f_{k_{i}}(\xi, \tau),
$$

for $j_{i}>2 k$. Thus, we deduce from (4.7) and the definition of $X_{k}$ that

$$
\left\|P_{k} \partial_{x}\left(u_{k_{1}} \partial_{x}^{2} v_{k_{2}}\right)\right\|_{N_{k}} \lesssim \sup _{t_{k} \in \mathbb{R}} 2^{3 k} \sum_{j, j_{1}, j_{2} \geq 2 k} 2^{-j / 2}\left\|\mathbf{1}_{D_{k, j}} \cdot f_{k_{1}, j_{1}} * f_{k_{2}, j_{2}}\right\|_{L_{\xi, \tau}^{2}},
$$

where $D_{k, j}$ is defined in (3.5). Here, we use that since $\left|\left(\tau-w(\xi)+i 2^{2 k}\right)^{-1}\right| \leq 2^{-2 k}$, the sum from $j=0$ to $2 k-1$ appearing implicitly on the right-hand side of (4.7) is controlled by the term corresponding to $j=2 k$ on the right-hand side of (4.8). Therefore, according to Corollary 2.7 and estimate (4.8) it suffices to prove that

$$
2^{3 k} \sum_{j \geq 2 k} 2^{-j / 2}\left\|\mathbf{1}_{D_{k, j}} \cdot\left(f_{k_{1}, j_{1}} * f_{k_{2}, j_{2}}\right)\right\|_{L_{\xi, \tau}^{2}} \lesssim 2^{j_{1} / 2}\left\|f_{k_{1}, j_{1}}\right\|_{L^{2}} 2^{j_{2} / 2}\left\|f_{k_{2}, j_{2}}\right\|_{L^{2}}
$$

with $j_{1}, j_{2} \geq 2 k$, in order to prove estimate (4.5). 
But, we deduce from estimates (3.29) and (3.31) that

$$
\begin{aligned}
2^{3 k} \sum_{j \geq 2 k} 2^{-j / 2} \| \mathbf{1}_{D_{k, j}} & \cdot\left(f_{k_{1}, j_{1}} * f_{k_{2}, j_{2}}\right) \|_{L_{\xi, \tau}^{2}} \\
& \lesssim 2^{3 k} \sum_{j \geq 2 k} 2^{-j / 2} 2^{-2 k} 2^{j_{1} / 2}\left\|f_{k_{1}, j_{1}}\right\|_{L^{2}} 2^{j_{2} / 2}\left\|f_{k_{2}, j_{2}}\right\|_{L^{2}},
\end{aligned}
$$

which implies estimate (4.9) after summing over $j$. This finishes the proof of Lemma 4.3 .

Lemma 4.6 (high $\times$ high $\rightarrow$ high). Assume that $k, k_{1}, k_{2} \in \mathbb{Z}_{+}$satisfy $k \geq 20$, $\left|k-k_{2}\right| \leq 5$ and $\left|k_{1}-k_{2}\right| \leq 5$. Then,

$$
\left\|P_{k} \partial_{x}\left(u_{k_{1}} \partial_{x}^{2} v_{k_{2}}\right)\right\|_{N_{k}} \lesssim\left\|u_{k_{1}}\right\|_{F_{k_{1}}}\left\|v_{k_{2}}\right\|_{F_{k_{2}}}
$$

and

$$
\left\|P_{k}\left(\partial_{x} u_{k_{1}} \partial_{x}^{2} v_{k_{2}}\right)\right\|_{N_{k}} \lesssim\left\|u_{k_{1}}\right\|_{F_{k_{1}}}\left\|v_{k_{2}}\right\|_{F_{k_{2}}},
$$

for all $u_{k_{1}} \in F_{k_{1}}$ and $v_{k_{2}} \in F_{k_{2}}$.

Proof. Once again we prove only estimate (4.10). Arguing as in the proof of Lemma 4.3 , it is enough to prove that

$$
2^{3 k} \sum_{j \geq 2 k} 2^{-j / 2}\left\|\mathbf{1}_{D_{k, j}} \cdot\left(f_{k_{1}, j_{1}} * f_{k_{2}, j_{2}}\right)\right\|_{L_{\xi, \tau}^{2}} \lesssim 2^{j_{1} / 2}\left\|f_{k_{1}, j_{1}}\right\|_{L^{2}} 2^{j_{2} / 2}\left\|f_{k_{2}, j_{2}}\right\|_{L^{2}},
$$

where $f_{k_{i}, j_{i}}$ is localized in $D_{k_{i}, j_{i}}$ with $j_{i} \geq 2 k$ for $i=1,2$.

We deduce by applying estimate (3.28) to the left-hand side of (4.12) that

$$
\begin{aligned}
2^{3 k} \sum_{j \geq 2 k} 2^{-j / 2} \| \mathbf{1}_{D_{k, j}} & \cdot\left(f_{k_{1}, j_{1}} * f_{k_{2}, j_{2}}\right) \|_{L_{\xi, \tau}^{2}} \\
& \lesssim 2^{3 k} \sum_{j \geq 2 k} 2^{-j / 2} 2^{k / 2} 2^{j_{m i n} / 2}\left\|f_{k_{1}, j_{1}}\right\|_{L^{2}}\left\|f_{k_{2}, j_{2}}\right\|_{L^{2}}
\end{aligned}
$$

According to Lemma 3.1 and the frequency localization, we have that

$$
2^{j_{\text {max }}} \sim \max \left\{2^{j_{\text {med }}}, 2^{5 k}\right\} .
$$

Finally, we observe that (4.13) and (4.14) imply estimate (4.12). This is clear in the cases where $j_{\max }=j_{1}$ or $j_{2}$ by using that $2^{j_{\max }} \gtrsim 2^{5 k}$ and summing over $j \geq 2 k$. In the case where $j_{\max }=j$, we have from (4.14) that either $2^{j} \sim 2^{5 k}$ or $2^{j} \sim 2^{j_{\text {med }}}$. When $2^{j} \sim 2^{5 k}$, estimate (4.12) follows directly from (4.13) since we do not need to sum over $j$, whereas when $2^{j} \sim 2^{j_{\text {med }}}$, we can use one of the cases $2^{j_{\text {max }}}=2^{j_{1}}$ or $2^{j_{\max }}=2^{j_{2}}$ to conclude.

Lemma 4.7 (high $\times$ high $\rightarrow$ low). Assume that $k, k_{1}, k_{2} \in \mathbb{Z}_{+}$satisfy $k_{2} \geq 20$, $\left|k_{1}-k_{2}\right| \leq 3$ and $0 \leq k \leq \max \left(k_{1}, k_{2}\right)-5$. Then,

$$
\begin{gathered}
\left\|P_{k} \partial_{x}\left(u_{k_{1}} \partial_{x}^{2} v_{k_{2}}\right)\right\|_{N_{k}} \lesssim k_{2} 2^{k_{2}-k}\left\|u_{k_{1}}\right\|_{F_{k_{1}}}\left\|v_{k_{2}}\right\|_{F_{k_{2}}}, \\
\left\|P_{k} \partial_{x}\left(\partial_{x} u_{k_{1}} \partial_{x} v_{k_{2}}\right)\right\|_{N_{k}} \lesssim k_{2} 2^{k_{2}-k}\left\|u_{k_{1}}\right\|_{F_{k_{1}}}\left\|v_{k_{2}}\right\|_{F_{k_{2}}},
\end{gathered}
$$

and

$$
\left\|P_{k}\left(\partial_{x} u_{k_{1}} \partial_{x}^{2} v_{k_{2}}\right)\right\|_{N_{k}} \lesssim k_{2} 2^{2\left(k_{2}-k\right)}\left\|u_{k_{1}}\right\|_{F_{k_{1}}}\left\|v_{k_{2}}\right\|_{F_{k_{2}}},
$$

for all $u_{k_{1}} \in F_{k_{1}}$ and $v_{k_{2}} \in F_{k_{2}}$. 
Remark 4.8. It is interesting to observe that the restriction $s>1$ in Proposition 4.1 appears in estimate (4.17).

Remark 4.9. Note that in the case $k=0$, by convention $P_{0}=P_{\leq 0}$.

Proof. We prove estimate (4.17), since estimates (4.15) and 4.16) could be proved in a similar way. Let $\gamma: \mathbb{R} \rightarrow[0,1]$ be a smooth function supported in $[-1,1]$ with the property that

$$
\sum_{m \in \mathbb{Z}} \gamma^{2}(x-m)=1, \quad \forall x \in \mathbb{R}
$$

We observe from the definition of $N_{k}$ in (2.6) that

$$
\begin{aligned}
\left\|P_{k} \partial_{x}\left(u_{k_{1}} \partial_{x}^{2} v_{k_{2}}\right)\right\|_{N_{k}} & \\
& \lesssim \sup _{t_{k} \in \mathbb{R}}\left\|\left(\tau-w(\xi)+i 2^{2 k}\right)^{-1} 2^{3 k_{2}} \mathbf{1}_{I_{k}} \sum_{|m| \lesssim 2^{2\left(k_{2}-k\right)}} f_{k_{1}}^{m} * f_{k_{2}}^{m}\right\|_{X_{k}},
\end{aligned}
$$

where

$$
f_{k_{1}}^{m}=\left|\mathcal{F}\left(\eta_{0}\left(2^{2 k}\left(\cdot-t_{k}\right)\right) \gamma\left(2^{2 k_{2}}\left(\cdot-t_{k}\right)-m\right) u_{k_{1}}\right)\right|
$$

and

$$
f_{k_{2}}^{m}=\left|\mathcal{F}\left(\widetilde{\eta}_{0}\left(2^{2 k}\left(\cdot-t_{k}\right)\right) \gamma\left(2^{2 k_{2}}\left(\cdot-t_{k}\right)-m\right) u_{k_{2}}\right)\right|,
$$

for $i=1,2$.

Now, we set

$$
f_{k_{i}, 2 k_{2}}^{m}(\xi, \tau)=\eta_{\leq 2 k_{2}}(\tau-w(\xi)) f_{k_{i}}^{m}(\xi, \tau) \text { and } f_{k_{i}, j_{i}}^{m}=\eta_{j_{i}}(\tau-w(\xi)) f_{k_{i}}^{m}(\xi, \tau),
$$

for $j_{i}>2 k_{2}$. Thus, we deduce from (4.18) and the definition of $X_{k}$ that

$$
\begin{aligned}
& \left\|P_{k} \partial_{x}\left(u_{k_{1}} \partial_{x}^{2} v_{k_{2}}\right)\right\|_{N_{k}} \\
& \quad \lesssim \sup _{t_{k} \in \mathbb{R}, m \in \mathbb{Z}} 2^{5 k_{2}} 2^{-2 k} \sum_{j \geq 0} \sum_{j_{1}, j_{2} \geq 2 k_{2}} 2^{-j / 2}\left\|\mathbf{1}_{D_{k, j}} \cdot f_{k_{1}, j_{1}}^{m} * f_{k_{2}, j_{2}}^{m}\right\|_{L_{\xi, \tau}^{2}}^{2} .
\end{aligned}
$$

Therefore, according to Lemma 2.2 and estimate (4.19) it suffices to prove that (4.20)

$$
2^{3 k_{2}} \sum_{j \geq 0} 2^{-j / 2}\left\|\mathbf{1}_{\widetilde{D}_{k, j}} \cdot\left(f_{k_{1}, j_{1}}^{m} * f_{k_{2}, j_{2}}^{m}\right)\right\|_{L_{\xi, \tau}^{2}} \lesssim k_{2} 2^{j_{1} / 2}\left\|f_{k_{1}, j_{1}}^{m}\right\|_{L^{2}} 2^{j_{2} / 2}\left\|f_{k_{2}, j_{2}}^{m}\right\|_{L^{2}},
$$

with $j_{1}, j_{2} \geq 2 k_{2}$, in order to prove estimate (4.17).

In the cases $j_{\max }=j_{1}$ or $j_{\max }=j_{2}$, say for example $j_{\max }=j_{1}$, we deduce from estimate (3.31) that

$$
\begin{aligned}
& 2^{3 k_{2}} \sum_{j \geq 0} 2^{-j / 2}\left\|\mathbf{1}_{\widetilde{D}_{k, j}} \cdot\left(f_{k_{1}, j_{1}}^{m} * f_{k_{2}, j_{2}}^{m}\right)\right\|_{L_{\xi, \tau}^{2}} \\
& \lesssim 2^{k_{2}} \sum_{j \geq 0} 2^{-j / 2} 2^{j / 2}\left\|f_{k_{1}, j_{1}}^{m}\right\|_{L^{2}} 2^{j_{2} / 2}\left\|f_{k_{2}, j_{2}}^{m}\right\|_{L^{2}} \\
& \lesssim k_{2} 2^{j_{1} / 2}\left\|f_{k_{1}, j_{1}}^{m}\right\|_{L^{2}} 2^{j_{2} / 2}\left\|f_{k_{2}, j_{2}}^{m}\right\|_{L^{2}}+2^{k_{2}} \sum_{j \geq 2 k_{2}} 2^{-j / 2} 2^{j_{1} / 2}\left\|f_{k_{1}, j_{1}}^{m}\right\|_{L^{2}} 2^{j_{2} / 2}\left\|f_{k_{2}, j_{2}}^{m}\right\|_{L^{2}},
\end{aligned}
$$

which implies estimate (4.19) by summing over $j$.

In the case $j_{\text {max }}=j$, we have that $2^{j} \sim \max \left\{2^{j_{\text {med }}},|\Omega|\right\}$, where $\Omega$ is defined in (3.1). If $2^{j} \sim 2^{j_{m e d}}$, then we are in one of the above cases, whereas in the case 
$2^{j} \sim|\Omega|$, we deduce from (3.2) that $j \leq 4 k_{2}+k+5$. Therefore, we get from (3.29) that

$$
\begin{aligned}
2^{3 k_{2}} \sum_{j \geq 0} 2^{-j / 2} \| \mathbf{1}_{\widetilde{D}_{k, j}} & \cdot\left(f_{k_{1}, j_{1}}^{m} * f_{k_{2}, j_{2}}^{m}\right) \|_{L_{\xi, \tau}^{2}} \\
& \lesssim 2^{k_{2}} k_{2} 2^{-j / 2} 2^{\left(j+j_{1}+j_{2}\right) / 2} 2^{-j_{m e d} / 2}\left\|f_{k_{1}, j_{1}}^{m}\right\|_{L^{2}}\left\|f_{k_{2}, j_{2}}^{m}\right\|_{L^{2}},
\end{aligned}
$$

which yields (4.19), since $j_{\text {med }} \geq 2 k_{2}$.

Lemma 4.10 (low $\times$ low $\rightarrow$ low). Assume that $k, k_{1}, k_{2} \in \mathbb{Z}_{+}$satisfy $0 \leq$ $k, k_{1}, k_{2} \leq 100$. Then,

$$
\left\|P_{k} \partial_{x}\left(u_{k_{1}} \partial_{x}^{2} v_{k_{2}}\right)\right\|_{N_{k}} \lesssim\left\|u_{k_{1}}\right\|_{F_{k_{1}}}\left\|v_{k_{2}}\right\|_{F_{k_{2}}}
$$

and

$$
\left\|P_{k}\left(\partial_{x} u_{k_{1}} \partial_{x}^{2} v_{k_{2}}\right)\right\|_{N_{k}} \lesssim\left\|u_{k_{1}}\right\|_{F_{k_{1}}}\left\|v_{k_{2}}\right\|_{F_{k_{2}}}
$$

for all $u_{k_{1}} \in F_{k_{1}}$ and $v_{k_{2}} \in F_{k_{2}}$.

Proof. Once again we prove only estimate (4.21). Arguing as in the proof of Lemma 4.3. it is enough to prove that

$$
\sum_{j \geq 0} 2^{-j / 2}\left\|\mathbf{1}_{D_{k, j}} \cdot\left(f_{k_{1}, j_{1}} * f_{k_{2}, j_{2}}\right)\right\|_{L_{\xi, \tau}^{2}} \lesssim 2^{j_{1} / 2}\left\|f_{k_{1}, j_{1}}\right\|_{L^{2}} 2^{j_{2} / 2}\left\|f_{k_{2}, j_{2}}\right\|_{L^{2}},
$$

where $f_{k_{i}, j_{i}}$ is localized in $D_{k_{i}, j_{i}}$ with $j_{i} \geq 0$ for $i=1$, 2, which is a direct consequence of estimate (3.28).

Finally, we give the proof of Proposition 4.1. Note that the proof of Proposition 4.2 would be similar.

Proof of Proposition 4.1. We only prove estimate (4.2), since the proof of estimate (4.1) would be similar. We choose two extensions $\tilde{u}$ and $\tilde{v}$ of $u$ and $v$ satisfying

$$
\|\tilde{u}\|_{F^{s}} \leq 2\|u\|_{F^{s}(T)} \quad \text { and } \quad\|\tilde{v}\|_{F^{s}} \leq 2\|v\|_{F^{s}(T)} .
$$

Therefore $\partial_{x} \tilde{u} \partial_{x}^{2} \tilde{v}$ is an extension of $\partial_{x} u \partial_{x}^{2} v$ on $\mathbb{R}^{2}$, and we have from the definition of $N^{s}(T)$ and Minkowski inequality that

$$
\left\|\partial_{x} u \partial_{x}^{2} v\right\|_{N^{s}(T)} \leq\left(\sum_{k \geq 0} 2^{2 k s}\left(\sum_{k_{1}, k_{2} \geq 0}\left\|P_{k}\left(\partial_{x} P_{k_{1}} \tilde{u} \partial_{x}^{2} P_{k_{2}} \tilde{v}\right)\right\|_{N_{k}}\right)^{2}\right)^{\frac{1}{2}},
$$

where we took the convention $P_{0}=P_{\leq 0}$. Moreover, we denote

$$
\begin{aligned}
& A_{1}=\left\{\left(k_{1}, k_{2}\right) \in \mathbb{Z}_{+}^{2}:\left|k_{2}-k\right| \leq 3 \text { and } 0 \leq k_{1} \leq \max \left(k, k_{2}\right)-5\right\}, \\
& A_{2}=\left\{\left(k_{1}, k_{2}\right) \in \mathbb{Z}_{+}^{2}:\left|k_{1}-k\right| \leq 3 \text { and } 0 \leq k_{2} \leq \max \left(k, k_{1}\right)-5\right\} \text {, } \\
& A_{3}=\left\{\left(k_{1}, k_{2}\right) \in \mathbb{Z}_{+}^{2}:\left|k_{1}-k_{2}\right| \leq 5,\left|k_{1}-k\right| \leq 5 \text { and } k \geq 20\right\} \text {, } \\
& A_{4}=\left\{\left(k_{1}, k_{2}\right) \in \mathbb{Z}_{+}^{2}:\left|k_{1}-k_{2}\right| \leq 3,0 \leq k \leq \max \left(k_{1}, k_{2}\right)-5 \text { and } k_{2} \geq 20\right\} \text {, } \\
& A_{5}=\left\{\left(k_{1}, k_{2}\right) \in \mathbb{Z}_{+}^{2}: 0 \leq k, k_{1}, k_{2} \leq 100\right\} .
\end{aligned}
$$

Note that for a given $k \in \mathbb{Z}_{+}$, some of these regions may be empty and others may overlap, but due to the frequency localization, we always have that

$$
\begin{aligned}
\left\|\partial_{x} u \partial_{x}^{2} v\right\|_{N^{s}(T)} & \lesssim \sum_{j=1}^{5}\left(\sum_{k \geq 0} 2^{2 k s}\left(\sum_{\left(k_{1}, k_{2}\right) \in A_{j}}\left\|P_{k}\left(\partial_{x} P_{k_{1}} \tilde{u} \partial_{x}^{2} P_{k_{2}} \tilde{v}\right)\right\|_{N_{k}}\right)^{2}\right)^{\frac{1}{2}} \\
& =: \sum_{j=1}^{5} S_{j} .
\end{aligned}
$$


To handle the sum $S_{1}$, we use estimate (4.6) and the Cauchy-Schwarz inequality to obtain that

$$
S_{1} \lesssim\left(\sum_{k \geq 0} 2^{2 k s}\left(\sum_{k_{1}=0}^{k-5}\left\|P_{k_{1}} \tilde{u}\right\|_{F_{k_{1}}}\left\|P_{k} \tilde{v}\right\|_{F_{k}}\right)^{2}\right)^{\frac{1}{2}} \lesssim\|\tilde{u}\|_{F^{0+}}\|\tilde{v}\|_{F^{s}},
$$

where we assumed without loss of generality that $\max \left(k, k_{2}\right)=k$. Similarly, we deduce from Remark 4.5 that

$$
S_{2} \lesssim\|\tilde{u}\|_{F^{s}}\|\tilde{v}\|_{F^{0+}}
$$

Estimate (4.11) leads to

$$
S_{3} \lesssim\left(\sum_{k \geq 0} 2^{2 k s}\left\|P_{k_{1}} \tilde{u}\right\|_{F_{k_{1}}}^{2}\left\|P_{k} \tilde{v}\right\|_{F_{k}}^{2}\right)^{\frac{1}{2}} \lesssim\|\tilde{u}\|_{F^{0}}\|\tilde{v}\|_{F^{s}} .
$$

Next, we deal with the sum $S_{4}$. Without loss of generality, assume that $\max \left(k_{1}, k_{2}\right)$ $=k_{2}$. It follows from estimate (4.17) and the Cauchy-Schwarz inequality in $k_{2}$ that

$$
\begin{aligned}
S_{4} & \lesssim\left(\sum_{k=0}^{k_{2}-5} 2^{2 k(s-2)}\left(\sum_{k_{2} \geq 20} k_{2} 2^{2 k_{2}}\left\|P_{k_{2}} \tilde{u}\right\|_{F_{k_{2}}} \sum_{\left|k^{\prime}-k_{2}\right| \leq 3}\left\|P_{k^{\prime}} \tilde{v}\right\|_{F_{k^{\prime}}}\right)^{2}\right)^{\frac{1}{2}} \\
& \lesssim\left(\sum_{k_{2} \geq 0} 2^{2 k_{2} s}\left\|P_{k_{2}} \tilde{u}\right\|_{F_{k_{2}}}^{2}\right)^{\frac{1}{2}}\left(\sum_{k_{2} \geq 0} 2^{2 k_{2}}\left\|P_{k_{2}} \tilde{v}\right\|_{F_{k_{2}}}^{2}\right)^{\frac{1}{2}} \\
& \lesssim\|\tilde{u}\|_{F^{s}}\|\tilde{v}\|_{F^{1}},
\end{aligned}
$$

since $s>1$. Finally, it is clear from estimate (4.22) that

$$
S_{5} \lesssim\|\tilde{u}\|_{F^{0}}\|\tilde{v}\|_{F^{0}}
$$

Therefore, we conclude the proof of estimate (4.2) by gathering (4.24)-(4.30).

\section{ENERGy Estimates}

As indicated in the introduction we assume for sake of simplicity that $c_{3}=0$. We also recall that, due to the short-time bilinear estimates derived in the last section, we need to work with $\alpha=2$ in the definition of the spaces $F_{\alpha}^{s}, F_{\alpha}^{s}(T)$ and $F_{k, \alpha}$, and therefore we will omit the index $\alpha=2$ to simplify the notation.

5.1. Energy estimates for a smooth solution. Due to the linear estimate (2.32), we need to control the norm $\|\cdot\|_{B^{s}(T)}$ of a solution $u$ to (1.1) as a function of $\left\|u_{0}\right\|_{H^{s}}$ and $\|u\|_{F^{s}(T)}$. However, we are not able to estimate $\|u\|_{B^{s}(T)}$ directly. We need to modify the energy by a cubic term to cancel some bad terms appearing after a commutator estimate (see Remark 5.1 below).

Let us define $\psi(\xi):=\xi \eta^{\prime}(\xi)$, where $\eta$ is defined in (2.3) and ' denotes the derivative, i.e. $\eta^{\prime}(\xi)=\frac{d}{d \xi} \eta(\xi)$. Then, for $k \geq 1$, we define $\psi_{k}(\xi)=\psi\left(2^{-k} \xi\right)$. We also denote by $Q_{k}$ the Littlewood-Paley multiplier of symbol $\psi_{k}$, i.e. $Q_{k} u=\mathcal{F}_{x}^{-1}\left(\psi_{k} \mathcal{F}_{x} u\right)$. From the definition of $\eta_{k}$ in (2.3), we observe that

$$
\psi_{k}(\xi)=2^{-k} \xi \eta^{\prime}\left(2^{-k} \xi\right)=\xi \frac{d}{d \xi}\left(\eta\left(2^{-k} \xi\right)\right)=\xi \eta_{k}^{\prime}(\xi)
$$


Finally, we define the new energy by

$$
\begin{aligned}
E_{k}(u)(t)= & \left\|P_{k} u(\cdot, t)\right\|_{L^{2}}^{2}+\alpha \int_{\mathbb{R}}\left(u P_{k} \partial_{x}^{-1} u Q_{k} \partial_{x}^{-1} u\right)(x, t) d x \\
& +\beta \int_{\mathbb{R}}\left(u P_{k} \partial_{x}^{-1} u P_{k} \partial_{x}^{-1} u\right)(x, t) d x,
\end{aligned}
$$

for any $k \geq 1$, and

$$
E_{T}^{s}(u)=\left\|P_{\leq 0} u(\cdot, 0)\right\|_{L^{2}}^{2}+\sum_{k \geq 1} 2^{2 k s} \sup _{t_{k} \in[-T, T]} E_{k}(u)\left(t_{k}\right),
$$

where $\alpha$ and $\beta$ are two real numbers which will be fixed later. This modified energy may be seen as a localized version of the one introduced by Kwon in [25].

Remark 5.1. The $L^{2}$-bilinear estimate: 8 do not permit us to control

$$
\left|\int_{\mathbb{R} \times[0, T]} P_{k} \partial_{x} v P_{k} \partial_{x} v P_{k_{1}} \partial_{x} u d x d t\right| \text { and }\left|\int_{\mathbb{R} \times[0, T]} P_{k} \partial_{x} v Q_{k} \partial_{x} v P_{k_{1}} \partial_{x} u d x d t\right|,
$$

which are lower-order terms appearing after a commutator estimate in Lemma 5.7 , without losing a $2^{k}$ factor. The modified energy in (5.3) is introduced to cancel those two terms after performing integrations by parts in the proof of Proposition 5.3 .

The next lemma states that when $\|u\|_{L_{T}^{\infty} H_{x}^{s}}$ is small, then $E_{T}^{s}(u)$ and $\|u\|_{B^{s}(T)}^{2}$ are comparable.

Lemma 5.2. Let $s>\frac{1}{2}$. Then, there exists $0<\delta_{0}$ such that

$$
\frac{1}{2}\|u\|_{B^{s}(T)}^{2} \leq E_{T}^{s}(u) \leq \frac{3}{2}\|u\|_{B^{s}(T)}^{2},
$$

for all $u \in B^{s}(T) \cap C\left([-T, T] ; H^{s}(\mathbb{R})\right)$ satisfying $\|u\|_{L_{T}^{\infty} H_{x}^{s}} \leq \delta_{0}$.

Proof. First observe that, due to the Sobolev embedding $H^{s}(\mathbb{R}) \hookrightarrow L^{\infty}(\mathbb{R})$,

$$
\left|\int_{\mathbb{R}}\left(u P_{k} \partial_{x}^{-1} u Q_{k} \partial_{x}^{-1} u\right)\left(x, t_{k}\right) d x\right| \lesssim\|u\|_{L_{T}^{\infty} H_{x}^{s}} \sum_{\left|k-k^{\prime}\right| \leq 3}\left\|P_{k^{\prime}} u\left(\cdot, t_{k}\right)\right\|_{L^{2}}^{2},
$$

for all $k \geq 1$. It follows that

$$
\begin{aligned}
E_{k}(u)(t) \geq & \left\|P_{k} u(t)\right\|_{L^{2}}^{2}-c|\alpha|\|u\|_{L_{T}^{\infty} H_{x}^{s}}\left\|P_{k}(u)(t)\right\|_{L^{2}} \sum_{\left|k-k^{\prime}\right| \leq 3}\left\|P_{k^{\prime}} u\left(\cdot, t_{k}\right)\right\|_{L^{2}}^{2} \\
& -c|\beta|\|u\|_{L_{T}^{\infty} H_{x}^{s}}\left\|P_{k}(u)(t)\right\|_{L^{2}}^{2},
\end{aligned}
$$

for any $t \in[-T, T]$ and $k \geq 1$. Thus, if we choose $\|u\|_{L_{T}^{\infty} H^{s}} \leq \delta_{0}$ with $\delta_{0}$ small enough, we obtain that

$$
\begin{aligned}
E_{k}(u)(t) \geq & \frac{3}{4}\left\|P_{k} u(t)\right\|_{L^{2}}^{2}-\frac{1}{50} \sup _{t_{k+1} \in[-T, T]}\left\|P_{k}(u)\left(t_{k+1}\right)\right\|_{L^{2}}^{2} \\
& -\frac{1}{50} \sup _{t_{k-1} \in[-T, T]}\left\|P_{k}(u)\left(t_{k-1}\right)\right\|_{L^{2}}^{2},
\end{aligned}
$$

which implies the first inequality in (5.4) after taking the supreme over $t \in[-T, T]$ and summing in $k \geq 1$. The second inequality in (5.4) follows similarly.

\footnotetext{
${ }^{7}$ In the form of Lemma 5.5 below.
} 
Proposition 5.3. Assume $s \geq 1$ and $T \in(0,1]$. Then, if $u \in C\left([-T, T] ; H^{\infty}(\mathbb{R})\right)$ is a solution to (1.1) with $c_{3}=0$, we have that

$$
\begin{aligned}
E_{T}^{s}(u) \lesssim & \left(1+\left\|u_{0}\right\|_{H^{s}}\right)\left\|u_{0}\right\|_{H^{s}}^{2}+\left(1+\|u\|_{F^{\frac{3}{4}}+(T)}\right)\|u\|_{F^{\frac{5}{4}}(T)}\|u\|_{F^{s}(T)}^{2} \\
& +\|u\|_{F^{\frac{3}{4}+}(T)}^{2}\|u\|_{B^{s}(T)}^{2} .
\end{aligned}
$$

As a corollary to Lemma 5.2 and Proposition 5.3, we deduce an a priori estimate in $\|\cdot\|_{B^{s}(T)}$ for smooth solutions to (1.1).

Corollary 5.4. Assume $s \geq 1$ and $T \in(0,1]$. Then, there exists $0<\delta_{0} \leq 1$ such that

$$
\|u\|_{B^{s}(T)}^{2} \lesssim\left\|u_{0}\right\|_{H^{s}}^{2}+\left(1+\|u\|_{F^{\frac{3}{4}+}(T)}\right)\|u\|_{F^{\frac{5}{4}(T)}}\|u\|_{F^{s}(T)}^{2}+\|u\|_{F^{\frac{3}{4}+}(T)}^{2}\|u\|_{B^{s}(T)}^{2},
$$

for all solutions $u$ to (1.1) with $c_{3}=0$ and satisfying $u \in C\left([-T, T] ; H^{\infty}(\mathbb{R})\right)$ and $\|u\|_{L_{T}^{\infty} H_{x}^{\frac{1}{2}+}}<\delta_{0}$.

We split the proof of Proposition 5.3 into several lemmas.

Lemma 5.5. Assume that $T \in(0,1], k_{1}, k_{2}, k_{3} \in \mathbb{Z}_{+}$and $u_{j} \in F_{k_{j}}$ for $j=1,2,3$.

(a) In the case $k_{\text {min }} \leq k_{\text {max }}-5$, it holds that

$$
\left|\int_{\mathbb{R} \times[0, T]} u_{1} u_{2} u_{3} d x d t\right| \lesssim 2^{-k_{\max }} \prod_{j=1}^{3}\left\|u_{j}\right\|_{F_{k_{j}}} .
$$

If moreover $k_{\text {min }} \geq 1$, we also have that

$$
\left|\int_{\mathbb{R} \times[0, T]} u_{1} u_{2} u_{3} d x d t\right| \lesssim 2^{-\frac{3}{2} k_{\max }} 2^{-k_{\min }} \prod_{j=1}^{3}\left\|u_{j}\right\|_{F_{k_{j}}} .
$$

(b) In the case $\left|k_{\min }-k_{\max }\right| \leq 10$, it holds that

$$
\left|\int_{\mathbb{R} \times[0, T]} u_{1} u_{2} u_{3} d x d t\right| \lesssim 2^{-7 k_{\max } / 4} \prod_{j=1}^{3}\left\|u_{j}\right\|_{F_{k_{j}}} .
$$

The following technical result will be needed in the proof of Lemma 5.5

Lemma 5.6. Assume $k \in \mathbb{Z}_{+}$and $I \subset \mathbb{R}$ is an interval. Then

$$
\sup _{j \in \mathbb{Z}_{+}} 2^{j / 2}\left\|\eta_{j}(\tau-w(\xi)) \mathcal{F}\left(\mathbf{1}_{I}(t) f\right)\right\|_{L^{2}} \lesssim\|\mathcal{F}(f)\|_{X_{k}},
$$

for all $f$ such that $\mathcal{F}(f) \in X_{k}$.

Proof. Fix $j \in \mathbb{Z}_{+}$. We can also assume that $j \geq 5$. By writing

$$
f=\sum_{q \geq 0} \mathcal{F}^{-1}\left[\eta_{q}(\tau-w(\xi)) \mathcal{F}(f)(\xi, \tau)\right]=: \sum_{q \geq 0} f_{q},
$$

we have that

$$
2^{j / 2}\left\|\eta_{j}(\tau-w(\xi)) \mathcal{F}\left(\mathbf{1}_{I}(t) f\right)\right\|_{L^{2}} \leq 2^{j / 2} \sum_{q \geq 0}\left\|\eta_{j}(\tau-w(\xi)) \mathcal{F}\left(\mathbf{1}_{I}(t) f_{q}\right)\right\|_{L^{2}}
$$

On the one hand, Plancherel's identity implies that

$$
2^{j / 2} \sum_{q \geq j-5}\left\|\eta_{j}(\tau-w(\xi)) \mathcal{F}\left(\mathbf{1}_{I}(t) f_{q}\right)\right\|_{L^{2}} \lesssim \sum_{q \geq j-5} 2^{q / 2}\left\|\eta_{q}(\tau-w(\xi)) \mathcal{F}(f)\right\|_{L^{2}}
$$


On the other hand, we have that $\left|\mathcal{F}_{t}\left(\mathbf{1}_{I}\right)(\tau)\right| \lesssim \frac{1}{|\tau|}$, since $I$ is an interval of $\mathbb{R}$. Thus, we deduce by applying the Cauchy-Schwarz inequality in $\tau^{\prime}$ that

$$
\begin{aligned}
2^{j / 2} \sum_{q=0}^{j-4} \| & \eta_{j}(\tau-w(\xi)) \mathcal{F}\left(\mathbf{1}_{I}(t) f_{q}\right) \|_{L^{2}} \\
& \lesssim 2^{j / 2} \sum_{q=0}^{j-4}\left\|\eta_{j}(\tau-w(\xi)) \int_{\mathbb{R}}\left|\mathcal{F}(f)\left(\xi, \tau^{\prime}\right)\right| \frac{\eta_{q}\left(\tau^{\prime}-w(\xi)\right)}{\left|\tau-\tau^{\prime}\right|} d \tau^{\prime}\right\|_{L_{\xi, \tau}^{2}} \\
& \lesssim \sum_{q=0}^{j-4} 2^{q / 2}\left\|\eta_{q}(\tau-w(\xi)) \mathcal{F}(f)\right\|_{L^{2}},
\end{aligned}
$$

since $\left|\tau-\tau^{\prime}\right| \sim 2^{j}$ in this case.

We deduce estimate (5.10) by gathering (5.11) -(5.13) and taking the supreme in $j$.

Proof of Lemma 5.5. Assume without loss of generality that $k_{1} \leq k_{2} \leq k_{3}$. Moreover, due to the frequency localization, we must have $\left|k_{2}-k_{3}\right| \leq 4$. We first prove estimate (5.7). Let $\beta: \mathbb{R} \rightarrow[0,1]$ be a smooth function supported in $[-1,1]$ with the property that

$$
\sum_{m \in \mathbb{Z}} \beta^{3}(x-m)=1, \quad \forall x \in \mathbb{R} .
$$

Then, it follows that

$$
\left|\int_{\mathbb{R} \times[0, T]} u_{1} u_{2} u_{3} d x d t\right| \lesssim \sum_{|m| \leq C 2^{2 k_{3}}}\left|\int_{\mathbb{R}^{2}} \prod_{i=1}^{3}\left(\beta\left(2^{2 k_{3}} t-m\right) \mathbf{1}_{[0, T]} u_{i}\right) d x d t\right|
$$

Now we observe that the sum on the right-hand side of (5.9) is taken over the two disjoint sets

$$
\mathcal{A}=\left\{m \in \mathbb{Z}: \beta\left(2^{2 k_{3}} t-m\right) \mathbf{1}_{[0, T]}=\beta\left(2^{2 k_{3}} t-m\right)\right\}
$$

and

$$
\mathcal{B}=\left\{m \in \mathbb{Z}: \beta\left(2^{2 k_{3}} t-m\right) \mathbf{1}_{[0, T]} \neq \beta\left(2^{2 k_{3}} t-m\right) \text { and } \beta\left(2^{2 k_{3}} t-m\right) \mathbf{1}_{[0, T]} \neq 0\right\} .
$$

To deal with the sum over $\mathcal{A}$, we set

$$
f_{k_{i}, 2 k_{3}}^{m}=\eta_{\leq 2 k_{3}}(\tau-w(\xi))\left|\mathcal{F}\left(\beta\left(2^{2 k_{3}} t-m\right) u_{i}\right)\right|
$$

and

$$
f_{k_{i}, j}^{m}=\eta_{j}(\tau-w(\xi))\left|\mathcal{F}\left(\beta\left(2^{2 k_{3}} t-m\right) u_{i}\right)\right|, \quad \text { for } j>2 k_{3}
$$

for each $m \in \mathcal{A}$ and $i \in\{1,2,3\}$. Therefore, we deduce by using Plancherel's identity and estimates (3.7), (3.9) that

$$
\begin{aligned}
\sum_{m \in \mathcal{A}} \mid \int_{\mathbb{R}^{2}} \prod_{i=1}^{3} & \left(\beta\left(2^{2 k_{3}} t-m\right) \mathbf{1}_{[-T, T]} u_{i}\right) d x d t \mid \\
& \lesssim \sup _{m \in \mathcal{A}} 2^{2 k_{3}} \sum_{j_{1}, j_{2}, j_{3} \geq 2 k_{3}} \int_{\mathbb{R}^{2}} f_{k_{1}, j_{1}}^{m} * f_{k_{2}, j_{2}}^{m} \cdot f_{k_{3}, j_{3}}^{m} d \xi d \tau \\
& \lesssim \sup _{m \in \mathcal{A}} 2^{-k_{3}} \prod_{i=1}^{3} \sum_{j_{i} \geq 2 k_{3}} 2^{j_{i} / 2}\left\|f_{k_{i}, j_{i}}^{m}\right\|_{L^{2}} .
\end{aligned}
$$


This implies, together with Lemma 2.2, that

$$
\sum_{m \in \mathcal{A}}\left|\int_{\mathbb{R}^{2}} \prod_{i=1}^{3}\left(\beta\left(2^{2 k_{3}} t-m\right) \mathbf{1}_{[0, T]} u_{i}\right) d x d t\right| \lesssim 2^{-k_{3}} \prod_{j=1}^{3}\left\|u_{j}\right\|_{F_{k_{j}}} .
$$

Now observe that $\# \mathcal{B} \leq 4$. We set

$$
g_{k_{i}, j}^{m}=\eta_{j}(\tau-w(\xi))\left|\mathcal{F}\left(\beta\left(2^{2 k_{3}} t-m\right) \mathbf{1}_{[0, T]} u_{i}\right)\right|,
$$

for $i \in\{1,2,3\}, j \geq 0$ and $m \in \mathcal{B}$. Then, we deduce by arguing as above and using Lemma 5.6 that

$$
\begin{aligned}
\sum_{m \in \mathcal{B}} \mid \int_{\mathbb{R}^{2}} \prod_{i=1}^{3} & \left(\beta\left(2^{2 k_{3}} t-m\right) \mathbf{1}_{[0, T]} u_{i}\right) d x d t \mid \\
& \lesssim \sup _{m \in \mathcal{B}} \sum_{j_{1}, j_{2}, j_{3} \geq 0} \int_{\mathbb{R}^{2}} g_{k_{1}, j_{1}}^{m} * g_{k_{2}, j_{2}}^{m} \cdot g_{k_{3}, j_{3}}^{m} d \xi d \tau \\
& \lesssim \sup _{m \in \mathcal{B}} 2^{-2 k_{3}} \sum_{j_{1}, j_{2}, j_{3} \geq 0} 2^{-j_{m e d} / 2} \prod_{i=1}^{3} \sup _{j_{i} \in \mathbb{Z}_{+}} 2^{j_{i} / 2}\left\|g_{k_{i}, j_{i}}^{m}\right\|_{L^{2}} \\
& \lesssim 2^{-2 k_{3}} k_{3} \prod_{j=1}^{3}\left\|u_{j}\right\|_{F_{k_{j}}} .
\end{aligned}
$$

Note that in the last step of (5.17), we use the fact that $2^{j_{\text {max }}} \sim \max \left(2^{j_{\text {med }}}, \Omega\right)$ to control the sum over $j_{\max }$. Indeed, the case $2^{j_{\max }} \sim 2^{j_{\text {med }}}$ is trivial, whereas in the case $2^{j_{\max }} \sim \Omega$, we observe from (3.2) that $j_{\max } \leq 5 k_{3}+6$.

We deduce estimate (5.7) by gathering (5.14) -(5.17). Note that estimate (5.8) is obtained arguing as in (5.14) (5.17) and by using (3.8) instead of (3.7) and the fact that $2^{j_{\max }} \gtrsim 2^{4 k_{3}} 2^{k_{1}}$ (cf. Lemma 3.1).

Finally, we give only a sketch of the proof of estimate (5.9), since it follows the same lines as the proof of estimate (5.7). Note that under the assumption $\left|k_{\text {min }}-k_{\text {med }}\right| \leq 4$, we have that $2^{k_{1}} \sim 2^{k_{2}} \sim 2^{k_{3}}$. Moreover, we can assume that $k_{1} \geq 10$, since the proof is trivial otherwise by using (3.6). We introduce the same decomposition as in (5.14) and split the summation domain in $\mathcal{A}$ and $\mathcal{B}$. The estimates for the sum over the regions $\mathcal{A}$ and $\mathcal{B}$ follow by using $(3.10)$ instead of (3.7) and (3.9) and the fact that $2^{j_{\max }} \gtrsim 2^{\frac{5}{2} k_{3}}$ (cf. Lemma 3.1).

Lemma 5.7. Assume that $T \in(0,1], k, k_{1} \in \mathbb{Z}_{+}$satisfy $k_{1} \leq k-6, u \in F_{k_{1}}$ and $v \in F^{0}$. Then, it holds that

$$
\begin{aligned}
& \mid \int_{\mathbb{R} \times[0, T]} P_{k} v P_{k} \partial_{x}\left(\partial_{x}^{2} v P_{k_{1}} u\right) d x d t \\
& -\frac{1}{2} \int_{\mathbb{R} \times[0, T]} P_{k} \partial_{x} v P_{k} \partial_{x} v P_{k_{1}} \partial_{x} u d x d t \\
& +\int_{\mathbb{R} \times[0, T]} P_{k} \partial_{x} v Q_{k} \partial_{x} v P_{k_{1}} \partial_{x} u d x d t \mid \lesssim \Theta\left(k, k_{1}\right)\left\|P_{k_{1}} u\right\|_{F_{k_{1}}} \sum_{\left|k^{\prime}-k\right| \leq 3}\left\|P_{k^{\prime}} v\right\|_{F_{k^{\prime}}}^{2}
\end{aligned}
$$


and

$$
\begin{aligned}
\mid \int_{\mathbb{R} \times[0, T]} P_{k} v P_{k}\left(P_{k_{1}} \partial_{x} u \partial_{x}^{2} v\right) & d x d t+\int_{\mathbb{R} \times[0, T]} P_{k} \partial_{x} v P_{k} \partial_{x} v P_{k_{1}} \partial_{x} u d x d t \mid \\
& \lesssim \Theta\left(k, k_{1}\right)\left\|P_{k_{1}} u\right\|_{F_{k_{1}}} \sum_{\left|k^{\prime}-k\right| \leq 3}\left\|P_{k^{\prime}} v\right\|_{F_{k^{\prime}}}^{2}
\end{aligned}
$$

where $\Theta\left(k, k_{1}\right)=2^{2 k_{1}}$. Moreover, if $k_{1} \geq 1$, we can choose $\Theta\left(k, k_{1}\right)=2^{k_{1}} 2^{-k / 2}$.

Proof of Lemma 5.7. We first prove estimate (5.18). After integrating by parts, we rewrite the term on the left-hand side of (5.18) as

$$
-\int_{\mathbb{R} \times[0, T]} P_{k} \partial_{x} v\left(\left[P_{k}, P_{k_{1}} u\right] \partial_{x}^{2} v-Q_{k} \partial_{x} v P_{k_{1}} \partial_{x} u\right) d x d t
$$

where $[A, B]=A B-B A$ denotes the commutator of $A$ and $B$. Now, straightforward computations using (5.1) lead to

$$
\begin{aligned}
& \mathcal{F}\left(\left[P_{k}, P_{k 1} u\right]\right.\left.\partial_{x}^{2} v-Q_{k} \partial_{x} v P_{k_{1}} \partial_{x} u\right)(\xi, \tau) \\
&=c \int_{\mathbb{R}^{2}} m\left(\xi, \xi_{1}\right) \mathcal{F}\left(P_{k_{1}} \partial_{x}^{2} u\right)\left(\xi_{1}, \tau_{1}\right) \mathcal{F}(v)\left(\xi-\xi_{1}, \tau-\tau_{1}\right) d \xi_{1} d \tau_{1},
\end{aligned}
$$

where

$$
\left|m\left(\xi, \xi_{1}\right)\right|:=\left|\frac{\eta_{k}(\xi)-\eta_{k}\left(\xi-\xi_{1}\right)-\eta_{k}^{\prime}\left(\xi-\xi_{1}\right) \xi_{1}}{\xi_{1}^{2}}\left(\xi-\xi_{1}\right)^{2}\right| \lesssim 1
$$

due to the Taylor-Lagrange theorem and the frequency localization on $\xi$ and $\xi_{1}$. Therefore estimate (5.18) follows arguing exactly as in the proof of Lemma 5.5.

To prove estimate (5.19), we first observe by integrating by parts that

$$
\begin{aligned}
\int_{\mathbb{R} \times[0, T]} P_{k} \partial_{x} v P_{k} \partial_{x} v P_{k_{1}} \partial_{x} u d x d t= & -\int_{\mathbb{R} \times[0, T]} P_{k} v P_{k} \partial_{x}^{2} v P_{k_{1}} \partial_{x} u d x d t \\
& -\int_{\mathbb{R} \times[0, T]} P_{k} v P_{k} \partial_{x} v P_{k_{1}} \partial_{x}^{2} u d x d t .
\end{aligned}
$$

First, we apply estimates (5.7) and (5.8) to obtain that

$$
\left|\int_{\mathbb{R} \times[0, T]} P_{k} v P_{k} \partial_{x} v P_{k_{1}} \partial_{x}^{2} u d x d t\right| \lesssim \theta\left(k, k_{1}\right)\left\|P_{k_{1}} u\right\|_{F_{k_{1}}}\left\|P_{k} v\right\|_{F_{k}}^{2} .
$$

On the other hand, we observe that

$$
\begin{aligned}
& \int_{\mathbb{R} \times[0, T]} P_{k} v P_{k}\left(\partial_{x}^{2} v P_{k_{1}} \partial_{x} u\right) d x d t-\int_{\mathbb{R} \times[0, T]} P_{k} v P_{k} \partial_{x}^{2} v P_{k_{1}} \partial_{x} u d x d t \\
& =\int_{\mathbb{R} \times[0, T]} P_{k} v\left(\left[P_{k}, P_{k_{1}} \partial_{x} u\right] \partial_{x}^{2} v\right) d x d t .
\end{aligned}
$$

An easy computation gives

$$
\begin{aligned}
& \mathcal{F}\left(\left[P_{k}, P_{k 1} \partial_{x} u\right] \partial_{x}^{2} v\right)(\xi, \tau) \\
& \quad=c \int_{\mathbb{R}^{2}} \widetilde{m}\left(\xi, \xi_{1}\right) \mathcal{F}\left(P_{k_{1}} \partial_{x}^{2} u\right)\left(\xi_{1}, \tau_{1}\right) \mathcal{F}\left(\partial_{x} v\right)\left(\xi-\xi_{1}, \tau-\tau_{1}\right) d \xi_{1} d \tau_{1},
\end{aligned}
$$

where

$$
\left|\widetilde{m}\left(\xi, \xi_{1}\right)\right|=\left|\frac{\eta_{k}(\xi)-\eta_{k}\left(\xi-\xi_{1}\right)}{\xi_{1}}\left(\xi-\xi_{1}\right)\right| \lesssim 1
$$


due to the mean value theorem and the frequency localization on $\xi$ and $\xi_{1}$. We finish the proof of estimate (5.19) arguing exactly as in the proof of Lemma 5.7.

Lemma 5.8. Assume that $T \in(0,1], k_{1}, k_{2}, k_{3}, k_{4} \in \mathbb{Z}_{+}$, and $u_{j} \in F_{k_{j}}$ for $j=1,2,3,4$. If $k_{t h d} \leq k_{\max }-5$, then it holds that

$$
\left|\int_{\mathbb{R} \times[0, T]} u_{1} u_{2} u_{3} u_{4} d x d t\right| \lesssim 2^{-k_{\max }} 2^{k_{\min } / 2} \prod_{j=1}^{4}\left\|u_{j}\right\|_{F_{k_{j}}} .
$$

If instead, $k_{\text {min }} \ll k_{\text {thd }} \sim k_{\text {sub }} \sim k_{\text {max }}$, then it holds that

$$
\left|\int_{\mathbb{R} \times[0, T]} u_{1} u_{2} u_{3} u_{4} d x d t\right| \lesssim 2^{-k_{\max }} 2^{k_{\min } / 2} \prod_{j=1}^{4}\left\|u_{j}\right\|_{F_{k_{j}}} .
$$

Proof. The proof of estimates (5.20) (5.21) follows by arguing exactly as in the proof of (5.7). To prove estimate (5.20), we use estimates (3.35)-(3.36) instead of estimates (3.7) and (3.9). To prove estimate (5.21), we use estimate (3.33) and observe that due to the frequency localization $\widetilde{\Omega} \sim 2^{5 k_{\max }}$, so that $j_{\max } \geq$ $5 k_{\max }-C$, where $C$ is a fixed positive constant depending only on the frequency localization and $\widetilde{\Omega}$ was defined in (3.32).

Now we give the proof of Proposition 5.3

Proof of Proposition 5.3. Let $u \in C\left([-T, T] ; H^{\infty}(\mathbb{R})\right)$ be a solution to (1.1) with $c_{3}=0$. We choose an extension $\widetilde{u}$ of $u$ on $\mathbb{R}^{2}$ satisfying

$$
\widetilde{u}_{\left.\right|_{\mathbb{R} \times[-T, T]}}=u \quad \text { and } \quad\|\widetilde{u}\|_{F^{s}} \leq 2\|u\|_{F^{s}(T)} .
$$

Then, for any $k \in \mathbb{Z}_{+} \cap[1,+\infty)$ and $t \in[-T, T]$, we differentiate $E_{k}(u)$ with respect to $t$ and deduce using (1.1) that

$$
\frac{d}{d t} E_{k}(u)=\mathcal{J}_{k}(u)+\mathcal{J}_{k}(u)+\alpha \mathcal{L}_{k}^{1}(u)+\alpha \mathcal{N}_{k}^{1}(u)+\beta \mathcal{L}_{k}^{2}(u)+\beta \mathcal{N}_{k}^{2}(u),
$$

where

$$
\begin{gathered}
\mathcal{J}_{k}(u)=2 c_{1} \int_{\mathbb{R}} P_{k} u P_{k} \partial_{x}\left(\left(\partial_{x} u\right)^{2}\right) d x \\
\partial_{k}(u)=2 c_{2} \int_{\mathbb{R}} P_{k} u P_{k} \partial_{x}\left(u \partial_{x}^{2} u\right) d x, \\
\mathcal{L}_{k}^{1}(u)=\int_{\mathbb{R}} \partial_{x}^{5} u P_{k} \partial_{x}^{-1} u Q_{k} \partial_{x}^{-1} u d x+\int_{\mathbb{R}} u P_{k} \partial_{x}^{4} u Q_{k} \partial_{x}^{-1} u d x \\
+\int_{\mathbb{R}} u P_{k} \partial_{x}^{-1} u Q_{k} \partial_{x}^{4} u d x, \\
\mathcal{N}_{k}^{1}(u)=c_{1} \int_{\mathbb{R}} \partial_{x}\left(\left(\partial_{x} u\right)^{2}\right) P_{k} \partial_{x}^{-1} u Q_{k} \partial_{x}^{-1} u d x+c_{1} \int_{\mathbb{R}} u P_{k}\left(\left(\partial_{x} u\right)^{2}\right) Q_{k} \partial_{x}^{-1} u d x \\
+c_{1} \int_{\mathbb{R}} u P_{k} \partial_{x}^{-1} u Q_{k}\left(\left(\partial_{x} u\right)^{2}\right) d x+c_{2} \int_{\mathbb{R}} \partial_{x}\left(u \partial_{x}^{2} u\right) P_{k} \partial_{x}^{-1} u Q_{k} \partial_{x}^{-1} u d x \\
+c_{2} \int_{\mathbb{R}} u P_{k}\left(u \partial_{x}^{2} u\right) Q_{k} \partial_{x}^{-1} u d x+c_{2} \int_{\mathbb{R}} u P_{k} \partial_{x}^{-1} u Q_{k}\left(u \partial_{x}^{2} u\right) d x, \\
\mathcal{L}_{k}^{2}(u)=\int_{\mathbb{R}} \partial_{x}^{5} u P_{k} \partial_{x}^{-1} u P_{k} \partial_{x}^{-1} u d x+2 \int_{\mathbb{R}} u P_{k} \partial_{x}^{4} u P_{k} \partial_{x}^{-1} u d x,
\end{gathered}
$$


and

$$
\begin{aligned}
\mathcal{N}_{k}^{2}(u)= & c_{1} \int_{\mathbb{R}} \partial_{x}\left(\left(\partial_{x} u\right)^{2}\right) P_{k} \partial_{x}^{-1} u P_{k} \partial_{x}^{-1} u d x+2 c_{1} \int_{\mathbb{R}} u P_{k}\left(\left(\partial_{x} u\right)^{2}\right) P_{k} \partial_{x}^{-1} u d x \\
& +c_{2} \int_{\mathbb{R}} \partial_{x}\left(u \partial_{x}^{2} u\right) P_{k} \partial_{x}^{-1} u P_{k} \partial_{x}^{-1} u d x+2 c_{2} \int_{\mathbb{R}} u P_{k}\left(u \partial_{x}^{2} u\right) P_{k} \partial_{x}^{-1} u d x .
\end{aligned}
$$

Now, we fix $t_{k} \in[-T, T]$. Without loss of generality, we can assume that $0<t_{k} \leq$ $T$. Therefore, we obtain by integrating (5.23) between 0 and $t_{k}$ that

$$
\begin{aligned}
& E_{k}(u)\left(t_{k}\right)-E_{k}(u)(0) \\
& \leq\left|\int_{\left[0, t_{k}\right]}\left(\mathcal{J}_{k}(u)+\mathcal{J}_{k}(u)+\alpha \mathcal{L}_{k}^{1}(u)+\alpha \mathcal{N}_{k}^{1}(u)+\beta \mathcal{L}_{k}^{2}(u)+\beta \mathcal{N}_{k}^{2}(u)\right) d t\right| .
\end{aligned}
$$

Next we estimate the right-hand side of (5.25).

Estimates for the cubic terms. We deduce after some integration by parts that

$$
\begin{aligned}
\mathcal{L}_{k}^{1}(u) & =\int_{\mathbb{R}} \partial_{x}^{3} u P_{k} \partial_{x} u Q_{k} \partial_{x}^{-1} u d x+2 \int_{\mathbb{R}} \partial_{x}^{3} u P_{k} u Q_{k} u d x+\int_{\mathbb{R}} \partial_{x}^{3} u P_{k} \partial_{x}^{-1} u Q_{k} \partial_{x} u d x \\
& +\int_{\mathbb{R}} \partial_{x}^{2} u P_{k} \partial_{x}^{2} u Q_{k} \partial_{x}^{-1} u d x+2 \int_{\mathbb{R}} \partial_{x} u P_{k} \partial_{x}^{2} u Q_{k} u d x+\int_{\mathbb{R}} u P_{k} \partial_{x}^{2} u Q_{k} \partial_{x} u d x \\
& +\int_{\mathbb{R}} \partial_{x}^{2} u P_{k} \partial_{x}^{-1} u Q_{k} \partial_{x}^{2} u d x+2 \int_{\mathbb{R}} \partial_{x} u P_{k} u Q_{k} \partial_{x}^{2} u d x+\int_{\mathbb{R}} u P_{k} \partial_{x} u Q_{k} \partial_{x}^{2} u d x \\
& =5 \int_{\mathbb{R}} \partial_{x}^{3} u P_{k} u Q_{k} u d x-5 \int_{\mathbb{R}} \partial_{x} u P_{k} \partial_{x} u Q_{k} \partial_{x} u d x .
\end{aligned}
$$

Similarly it holds that

$$
\mathcal{L}_{k}^{2}(u)=5 \int_{\mathbb{R}} \partial_{x}^{3} u P_{k} u P_{k} u d x-5 \int_{\mathbb{R}} \partial_{x} u P_{k} \partial_{x} u P_{k} \partial_{x} u d x .
$$

We choose $\alpha=-\frac{2 c_{2}}{5}$ and $\beta=\frac{c_{2}-4 c_{1}}{5}$. Then it follows, after performing a dyadic decomposition on $u$, that

$$
\left|\int_{\left[0, t_{k}\right]}\left(\mathcal{J}_{k}(u)+\mathcal{J}_{k}(u)+\alpha \mathcal{L}_{k}^{1}(u)+\beta \mathcal{L}_{k}^{2}(u)\right) d t\right| \lesssim \sum_{j=1}^{7} T_{j}(k),
$$

for each $k \geq 1$, with

$$
\begin{gathered}
T_{1}(k)=\sum_{0 \leq k_{1} \leq k-6}\left|\int_{\mathbb{R} \times\left[0, t_{k}\right]}\left(P_{k} u P_{k}\left(\partial_{x}^{2} u P_{k_{1}} \partial_{x} u\right)+P_{k} \partial_{x} u P_{k} \partial_{x} u P_{k_{1}} \partial_{x} u\right) d x d t\right|, \\
T_{2}(k)=\sum_{0 \leq k_{1} \leq k-6}\left|\int_{\mathbb{R} \times\left[0, t_{k}\right]} P_{k} \partial_{x} u\left(\left[P_{k}, P_{k_{1}} u\right] \partial_{x}^{2} u-Q_{k} \partial_{x} u P_{k_{1}} \partial_{x} u\right) d x d t\right|, \\
T_{3}(k)=\sum_{k_{1} \geq k-5, k_{2} \geq 0}\left|\int_{\mathbb{R} \times\left[0, t_{k}\right]} P_{k}^{2} u P_{k_{1}} \partial_{x} u P_{k_{2}} \partial_{x}^{2} u d t\right|, \\
T_{4}(k)=\sum_{k_{1} \geq k-5, k_{2} \geq 0}\left|\int_{\mathbb{R} \times\left[0, t_{k}\right]} P_{k}^{2} \partial_{x} u P_{k_{1}} u P_{k_{2}} \partial_{x}^{2} u d t\right|, \\
T_{5}(k)=\sum_{k-5 \leq k_{1} \leq k+4}\left|\int_{\mathbb{R} \times\left[0, t_{k}\right]} P_{k_{1}} \partial_{x} u P_{k} \partial_{x} u\left(Q_{k} \partial_{x} u+P_{k} \partial_{x} u\right) d x d t\right|,
\end{gathered}
$$




$$
T_{6}(k)=\sum_{k_{1} \leq k-5}\left|\int_{\mathbb{R} \times\left[0, t_{k}\right]} P_{k_{1}} \partial_{x}^{3} u P_{k} u\left(Q_{k} u+P_{k} u\right) d x\right|
$$

and

$$
T_{7}(k)=\sum_{k-4 \leq k_{1} \leq k+4}\left|\int_{\mathbb{R} \times\left[0, t_{k}\right]} P_{k_{1}} \partial_{x}^{3} u P_{k} u\left(Q_{k} u+P_{k} u\right) d x\right| .
$$

Clearly, Lemma 5.7 and the Cauchy-Schwarz inequality imply that

$$
\begin{aligned}
T_{1}(k)+T_{2}(k) & \lesssim \sum_{0 \leq k_{1} \leq k-6} 2^{k_{1} / 2}\left\|P_{k_{1}} \widetilde{u}\right\|_{F_{k_{1}}} \sum_{\left|k-k^{\prime}\right| \leq 3}\left\|P_{k^{\prime}} \widetilde{u}\right\|_{F_{k^{\prime}}}^{2} \\
& \lesssim\|\widetilde{u}\|_{F^{1 / 2+}} \sum_{\left|k-k^{\prime}\right| \leq 3}\left\|P_{k^{\prime}} \widetilde{u}\right\|_{F_{k^{\prime}}}^{2} .
\end{aligned}
$$

Similarly, we get by applying estimate (5.7) if $k_{1}=0$ and estimate (5.8) if $k_{1}>0$ that

$$
T_{6}(k) \lesssim\|\widetilde{u}\|_{F^{\frac{1}{2}+}} \sum_{\left|k-k^{\prime}\right| \leq 3}\left\|P_{k^{\prime}} \widetilde{u}\right\|_{F_{k^{\prime}}}^{2} .
$$

Now, estimate (5.9) leads to

$$
T_{5}(k)+T_{7}(k) \lesssim\|\widetilde{u}\|_{F^{\frac{5}{4}}}\left\|P_{k} \widetilde{u}\right\|_{F_{k}}^{2} .
$$

To estimate $T_{3}(k)$, when $k \geq 1$ is given, we denote

$$
\begin{aligned}
& B_{1}=\left\{\left(k, k_{1}\right) \in \mathbb{Z}_{+}^{2}:\left|k-k_{1}\right| \leq 3 \text { and } 0 \leq k_{2} \leq \max \left(k, k_{1}\right)-5\right\}, \\
& B_{2}=\left\{\left(k, k_{1}\right) \in \mathbb{Z}_{+}^{2}:\left|k-k_{1}\right| \leq 5 \text { and }\left|k_{2}-k_{1}\right| \leq 5\right\} \text {, } \\
& B_{3}=\left\{\left(k, k_{1}\right) \in \mathbb{Z}_{+}^{2}:\left|k_{2}-k_{1}\right| \leq 3 \text { and } 1 \leq k \leq \max \left(k_{1}, k_{2}\right)-5\right\} \text {. }
\end{aligned}
$$

Thus, we deduce from the frequency localization that

$$
T_{3}(k)=\sum_{j=1}^{3} \sum_{\left(k_{1}, k_{2}\right) \in B_{j}}\left|\int_{\mathbb{R} \times\left[0, t_{k}\right]} P_{k}^{2} u P_{k_{1}} \partial_{x} u P_{k_{2}} \partial_{x}^{2} u d t\right| .
$$

To estimate the sum over $B_{1}$, we use estimate (5.7) in the case $k_{2}=0$ and estimate (5.8) in the case $k_{2} \geq 1$. It follows that

$$
\sum_{\left(k_{1}, k_{2}\right) \in B_{1}}\left|\int_{\mathbb{R} \times\left[0, t_{k}\right]} P_{k}^{2} u P_{k_{1}} \partial_{x} u P_{k_{2}} \partial_{x}^{2} u d t\right| \lesssim\|\widetilde{u}\|_{F^{\frac{1}{2}+}} \sum_{\left|k^{\prime}-k\right| \leq 3}\left\|P_{k^{\prime}} \widetilde{u}\right\|_{F_{k^{\prime}}}^{2} .
$$

The sum over $B_{2}$ is treated by using estimate (5.9), which gives

$$
\sum_{\left(k_{1}, k_{2}\right) \in B_{2}}\left|\int_{\mathbb{R} \times\left[0, t_{k}\right]} P_{k}^{2} u P_{k_{1}} \partial_{x} u P_{k_{2}} \partial_{x}^{2} u d t\right| \lesssim\|\widetilde{u}\|_{F^{\frac{5}{4}}} \sum_{\left|k^{\prime}-k\right| \leq 3}\left\|P_{k^{\prime}} \widetilde{u}\right\|_{F_{k^{\prime}}}^{2}
$$

Finally, estimate (5.8) (recall here that $k \geq 1$ ) yields

$$
\sum_{\left(k_{1}, k_{2}\right) \in B_{3}}\left|\int_{\mathbb{R} \times\left[0, t_{k}\right]} P_{k}^{2} u P_{k_{1}} \partial_{x} u P_{k_{2}} \partial_{x}^{2} u d t\right| \lesssim 2^{-k}\left\|P_{k} \widetilde{u}\right\|_{F_{k}} \sum_{k_{1} \geq k+5} 2^{3 k_{1} / 2}\left\|P_{k_{1}} \widetilde{u}\right\|_{F_{k_{1}}}^{2} .
$$

Moreover, observe that the same estimates also hold for $T_{4}(k)$ (with an even better bound when the sum is taken over $\left.B_{3}\right)$. This implies that

$$
\begin{aligned}
T_{3}(k)+T_{4}(k) \lesssim & \|\widetilde{u}\|_{F^{\frac{5}{4}}} \sum_{\left|k^{\prime}-k\right| \leq 3}\left\|P_{k^{\prime}} \widetilde{u}\right\|_{F_{k}}^{2} \\
& +2^{-k}\left\|P_{k} \widetilde{u}\right\|_{F_{k}} \sum_{k_{1} \geq k+5} 2^{3 k_{1} / 2}\left\|P_{k_{1}} \widetilde{u}\right\|_{F_{k_{1}}}^{2} .
\end{aligned}
$$


Therefore, we deduce by gathering (5.26) (5.30), taking the supreme over $t_{k} \in$ $[0, T]$, summing in $k$ and using (5.22) that

$$
\begin{aligned}
& \sum_{k \geq 1} 2^{2 k s} \sup _{t_{k} \in[0, T]}\left|\int_{\left[0, t_{k}\right]} n\left(\mathcal{J}_{k}(u)+\mathcal{J}_{k}(u)+\alpha \mathcal{L}_{k}^{1}(u)+\beta \mathcal{L}_{k}^{2}(u)\right) d t\right| \\
& \lesssim \sum_{k \geq 1} 2^{2 k s}\|\widetilde{u}\|_{F^{\frac{5}{4}}}\left\|P_{k} \widetilde{u}\right\|_{F_{k}}^{2}+\sum_{k \geq 1} 2^{k(2 s-1)}\left\|P_{k} \widetilde{u}\right\|_{F_{k}} \sum_{k_{1} \geq k+5} 2^{3 k_{1} / 2}\left\|P_{k_{1}} \widetilde{u}\right\|_{F_{k_{1}}}^{2} \\
& \lesssim\|u\|_{F^{\frac{5}{4}(T)}}\|u\|_{F^{s}(T)}^{2}+\|u\|_{F^{\frac{1}{2}+}(T)}\|u\|_{F^{s}(T)}^{2} .
\end{aligned}
$$

Note that we use that $s \geq 1$ and apply the Cauchy-Schwarz inequality in $k$ and $k_{1}$ to obtain the last inequality in (5.31).

Estimates for the fourth-order terms. We estimate the fourth-order term corresponding to $\mathcal{N}_{k}^{2}(u)$. After some integration by parts in (5.24), we get that

$$
\left|\int_{\left[0, t_{k}\right]} \mathcal{N}_{k}^{2}(u) d t\right| \lesssim \sum_{i=1}^{4} X_{i}(k),
$$

for each $k \geq 1$, with

$$
\begin{gathered}
X_{1}(k)=\left|\int_{\mathbb{R} \times\left[0, t_{k}\right]}\left(\partial_{x} u\right)^{2} P_{k} \partial_{x}^{-1} u P_{k} u d x d t\right|+\left|\int_{\mathbb{R} \times\left[0, t_{k}\right]} u \partial_{x} u P_{k} u P_{k} u d x d t\right| \\
+\left|\int_{\mathbb{R} \times\left[0, t_{k}\right]} u \partial_{x} u P_{k} \partial_{x} u P_{k} \partial_{x}^{-1} u d x d t\right|, \\
X_{2}(k)=\left|\int_{\mathbb{R} \times\left[0, t_{k}\right]} u P_{k}\left(\left(\partial_{x} u\right)^{2}\right) P_{k} \partial_{x}^{-1} u d x d t\right|, \\
X_{3}(k)=\left|\int_{\mathbb{R} \times\left[0, t_{k}\right]} \partial_{x} u P_{k}\left(u \partial_{x} u\right) P_{k} \partial_{x}^{-1} u d x d t\right|
\end{gathered}
$$

and

$$
X_{4}(k)=\left|\int_{\mathbb{R} \times\left[0, t_{k}\right]} u P_{k}\left(u \partial_{x} u\right) P_{k} u d x d t\right| .
$$

We use the Strichartz estimate (2.47) with $\alpha=2$, estimate (2.10) and Hölder's inequality to deduce that

$$
\begin{aligned}
\sum_{k \geq 1} 2^{2 k s} \sup _{t_{k} \in[0, T]} X_{1}(k) & \lesssim\left(\|u\|_{L_{T}^{2} L_{x}^{\infty}}+\left\|\partial_{x} u\right\|_{L_{T}^{2} L_{x}^{\infty}}\right)\left\|\partial_{x} u\right\|_{L_{T}^{2} L_{x}^{\infty}} \sum_{k \geq 1} 2^{2 k s}\left\|P_{k} u\right\|_{L_{T}^{\infty} L_{x}^{2}}^{2} \\
& \lesssim\left(\|u\|_{F^{\frac{1}{2}+}(T)}+\|u\|_{F^{\frac{3}{4}+}(T)}\right)\|u\|_{F^{\frac{3}{4}+}(T)}\|u\|_{B^{s}(T)}^{2} .
\end{aligned}
$$

To deal with $X_{2}(k)$, we perform dyadic decompositions over $u$ and $\partial_{x} u$. Then

$$
X_{2}(k) \leq \sum_{j=1}^{4} \sum_{\left(k_{1}, k_{2}\right) \in D_{j}}\left|\int_{\mathbb{R} \times\left[0, t_{k}\right]} u P_{k}\left(P_{k_{1}} \partial_{x} u P_{k_{2}} \partial_{x} u\right) P_{k} \partial_{x}^{-1} u d x d t\right|,
$$

where

$$
\begin{aligned}
& D_{1}=\left\{\left(k_{1}, k_{2}\right) \in \mathbb{Z}_{+}^{2}:\left|k-k_{1}\right| \leq 3 \text { and } 0 \leq k_{2} \leq \max \left(k, k_{1}\right)-5\right\} \text {, } \\
& D_{2}=\left\{\left(k_{1}, k_{2}\right) \in \mathbb{Z}_{+}^{2}:\left|k-k_{2}\right| \leq 3 \text { and } 0 \leq k_{1} \leq \max \left(k, k_{2}\right)-5\right\} \text {, } \\
& D_{3}=\left\{\left(k_{1}, k_{2}\right) \in \mathbb{Z}_{+}^{2}:\left|k-k_{1}\right| \leq 5 \text { and }\left|k_{2}-k_{1}\right| \leq 5\right\} \text {, } \\
& D_{4}=\left\{\left(k_{1}, k_{2}\right) \in \mathbb{Z}_{+}^{2}:\left|k_{2}-k_{1}\right| \leq 3 \text { and } 1 \leq k \leq \max \left(k_{1}, k_{2}\right)-5\right\} \text {. }
\end{aligned}
$$


By using Hölder's inequality and the Cauchy-Schwarz inequality, we can bound the sum over $D_{1} \cup D_{2}$ by

$$
\|u\|_{L_{T, x}^{\infty}}\left\|D_{x}^{1+} u\right\|_{L_{T}^{2} L_{x}^{\infty}} \sum_{\left|k^{\prime}-k\right| \leq 3}\left\|P_{k^{\prime}} u\right\|_{L_{T}^{\infty} L_{x}^{2}}^{2} .
$$

Thus, it follows from estimates (2.10) and (2.48) that

$$
\begin{aligned}
\sum_{\left(k_{1}, k_{2}\right) \in D_{1} \cup D_{2}}\left|\int_{\mathbb{R} \times\left[0, t_{k}\right]} u P_{k}\left(P_{k_{1}} \partial_{x} u P_{k_{2}} \partial_{x} u\right) P_{k} \partial_{x}^{-1} u d x d t\right| \\
\lesssim\|u\|_{F^{\frac{1}{2}+}(T)}\|u\|_{F^{\frac{3}{4}+}{ }^{+}(T)} \sum_{\left|k^{\prime}-k\right| \leq 3}\left\|P_{k^{\prime}} u\right\|_{L_{T}^{\infty} L_{x}^{2}}^{2} .
\end{aligned}
$$

A similar bound holds over $D_{3}$. In the region $D_{4}$, we have that

$$
\begin{aligned}
\sum_{\left(k_{1}, k_{2}\right) \in D_{4}} \mid & \int_{\mathbb{R} \times\left[0, t_{k}\right]} u P_{k}\left(P_{k_{1}} \partial_{x} u P_{k_{2}} \partial_{x} u\right) P_{k} \partial_{x}^{-1} u d x d t \mid \\
& \lesssim\|u\|_{F^{\frac{1}{2}+}(T)} 2^{-k}\left\|P_{k} u\right\|_{L_{T}^{\infty} L_{x}^{2}} \sum_{k_{1} \geq k+5} 2^{2 k_{1}}\left\|P_{k_{1}} u\right\|_{L_{T}^{2} L_{x}^{\infty}}\left\|P_{k_{1}} u\right\|_{L_{T}^{\infty} L_{x}^{2}}
\end{aligned}
$$

Hence, we deduce after taking the supreme of $t_{k}$ over $[0, T]$, summing over $k \in$ $\mathbb{Z}_{+} \cap[1,+\infty)$ and using estimate (2.48) that

$$
\sum_{k \geq 1} 2^{2 k s} \sup _{t_{k} \in[0, T]} X_{2}(k) \lesssim\|u\|_{F^{\frac{1}{2}+}(T)}\|u\|_{F^{\frac{3}{4}+}(T)}\|u\|_{B^{s}(T)}^{2} .
$$

Similarly, we get that

$$
\sum_{k \geq 1} 2^{2 k s} \sup _{t_{k} \in[0, T]} X_{3}(k) \lesssim\|u\|_{F^{\frac{3}{4}+}(T)}^{2}\|u\|_{B^{s}(T)}^{2} .
$$

To deal with $X_{4}(k)$, we use the following decomposition:

$$
\begin{aligned}
X_{4}(k) \leq & \sum_{k_{1} \geq k-7}\left|\int_{\mathbb{R} \times\left[0, t_{k}\right]} P_{k_{1}} u P_{k}\left(u \partial_{x} u\right) P_{k} u d x d t\right| \\
& +\sum_{j=1}^{4} \sum_{\left(k_{1}, k_{2}, k_{3}\right) \in E_{j}}\left|\int_{\mathbb{R} \times\left[0, t_{k}\right]} P_{k_{1}} u P_{k}\left(P_{k_{2}} u \partial_{x} P_{k_{3}} u\right) P_{k} u d x d t\right| \\
= & : \sum_{j=0}^{4} X_{4, j}(k),
\end{aligned}
$$

where

$$
\begin{aligned}
& E_{1}=\left\{\left(k_{1}, k_{2}, k_{3}\right) \in \mathbb{Z}_{+}^{3}: 0 \leq k_{1} \leq k-8,\left|k-k_{3}\right| \leq 3,0 \leq k_{2} \leq \max \left(k, k_{3}\right)-5\right\} \text {, } \\
& E_{2}=\left\{\left(k_{1}, k_{2}, k_{3}\right) \in \mathbb{Z}_{+}^{3}: 0 \leq k_{1} \leq k-8,\left|k-k_{2}\right| \leq 3,0 \leq k_{3} \leq \max \left(k, k_{2}\right)-5\right\} \text {, } \\
& E_{3}=\left\{\left(k_{1}, k_{2}, k_{3}\right) \in \mathbb{Z}_{+}^{3}: 0 \leq k_{1} \leq k-8,\left|k-k_{2}\right| \leq 5,\left|k-k_{3}\right| \leq 5\right\} \text {, } \\
& E_{4}=\left\{\left(k_{1}, k_{2}, k_{3}\right) \in \mathbb{Z}_{+}^{3}: 0 \leq k_{1} \leq k-8,\left|k_{2}-k_{3}\right| \leq 3,1 \leq k \leq \max \left(k_{2}, k_{3}\right)-5\right\} .
\end{aligned}
$$

Observe that, according to estimates (2.10) and (2.47),

$$
\begin{aligned}
X_{4,0}(k) & =\sum_{k-7 \leq k_{1} \leq k+3}\left|\int_{\mathbb{R} \times\left[0, t_{k}\right]} u \partial_{x} u P_{k}\left(P_{k_{1}} u P_{k} u\right) d x d t\right| \\
& \lesssim\|u\|_{F^{\frac{1}{2}+}(T)}\|u\|_{F^{\frac{3}{4}+}{ }^{(T)}} \sum_{\left|k^{\prime}-k\right| \leq 7}\left\|P_{k^{\prime}} u\right\|_{L_{T}^{\infty} L_{x}^{2} .}^{2}
\end{aligned}
$$


Now, by using estimate (5.20), we get that

$$
X_{4,1}(k)+X_{4,2}(k) \lesssim\|\widetilde{u}\|_{F^{\frac{1}{2}+}}\|\widetilde{u}\|_{F^{0+}} \sum_{\left|k^{\prime}-k\right| \leq 3}\left\|P_{k^{\prime}} \widetilde{u}\right\|_{F_{k^{\prime}}}^{2} .
$$

Over the region $E_{3}$, we deduce from estimates (2.10) and (2.48) that

$$
X_{4,3}(k) \lesssim\|u\|_{F^{\frac{1}{2}+}(T)}\|u\|_{F^{\frac{3}{4}+}(T)} \sum_{\left|k^{\prime}-k\right| \leq 5}\left\|P_{k^{\prime}} u\right\|_{L_{T}^{\infty} L_{x}^{2}}^{2} .
$$

Finally, estimate (2.10) gives

$$
X_{4,4}(k) \lesssim\|u\|_{F^{\frac{1}{2}+}(T)}\left\|P_{k} u\right\|_{L_{T}^{\infty} L_{x}^{2}} \sum_{k_{3} \geq k+5}\left\|P_{k_{3}} u\right\|_{L_{T}^{\infty} L_{x}^{2}}\left\|P_{k_{3}} \partial_{x} u\right\|_{L_{T}^{2} L_{x}^{\infty}} .
$$

Thus, we deduce from (5.37) - (5.41) that

$$
\sum_{k \geq 1} 2^{2 k s} \sup _{t_{k} \in[0, T]} X_{4}(k) \lesssim\|u\|_{F^{\frac{3}{4}+}(T)}^{2}\|u\|_{B^{s}(T)}^{2}+\|u\|_{F^{0+}(T)}\|u\|_{F^{\frac{1}{2}+}(T)}\|u\|_{F^{s}(T)}^{2} .
$$

Therefore, we conclude by gathering (5.32) -(5.36) and (5.42) that

$$
\begin{aligned}
\sum_{k \geq 1} 2^{2 k s} \sup _{t_{k} \in[0, T]} \mid & \int_{\left[0, t_{k}\right]} \mathcal{N}_{k}^{2}(u) d t \mid \\
& \lesssim\|u\|_{F^{\frac{3}{4}+}(T)}^{2}\|u\|_{B^{s}(T)}^{2}+\|u\|_{F^{0+}(T)}\|u\|_{F^{\frac{1}{2}+}(T)}\|u\|_{F^{s}(T)}^{2} .
\end{aligned}
$$

By using the same arguments, we could obtain a similar bound for $\mathcal{N}_{k}^{1}(u)$.

We finish the proof of Proposition 5.3 by recalling the definition of the energy in (5.3) and gathering estimates (5.25), (5.31) and (5.43).

5.2. Energy estimates for the differences of two solutions. In this subsection, we assume that $s \geq 2$. Let $u_{1}$ and $u_{2}$ be two solutions to the equation (1.1) with $c_{3}=0$ in the class (1.9) satisfying $u_{1}(\cdot, 0)=\varphi_{1}$ and $u_{2}(\cdot, 0)=\varphi_{2}$. Then by setting $v=u_{1}-u_{2}$, we see that $v$ must satisfy

$$
\partial_{t} v=\partial_{x}^{5} v+2 c_{1} \partial_{x} u_{1} \partial_{x}^{2} v+2 c_{1} \partial_{x} v \partial_{x}^{2} u_{2}+c_{2} \partial_{x}\left(u_{1} \partial_{x}^{2} v\right)+c_{2} \partial_{x}\left(v \partial_{x}^{2} u_{2}\right),
$$

with $v(\cdot, 0)=\varphi:=\varphi_{1}-\varphi_{2}$. As in subsection 5.1] we introduce the energy $\widetilde{E}_{T}^{s}(v)$ associated to (5.44). For $k \geq 1$,

$$
\begin{aligned}
\widetilde{E}_{k}(v)(t)= & \left\|P_{k} v(\cdot, t)\right\|_{L^{2}}^{2}+\widetilde{\alpha} \int_{\mathbb{R}}\left(u_{1} P_{k} \partial_{x}^{-1} v Q_{k} \partial_{x}^{-1} v\right)(x, t) d x \\
& +\widetilde{\beta} \int_{\mathbb{R}}\left(u_{1} P_{k} \partial_{x}^{-1} v P_{k} \partial_{x}^{-1} v\right)(x, t) d x
\end{aligned}
$$

and

$$
\widetilde{E}_{T}^{s}(v)=\left\|P_{\leq 0} v(\cdot, 0)\right\|_{L^{2}}^{2}+\sum_{k \geq 1} 2^{2 k s} \sup _{t_{k} \in[-T, T]} \widetilde{E}_{k}(v)\left(t_{k}\right),
$$

where $\widetilde{\alpha}$ and $\widetilde{\beta}$ are two real numbers which will be fixed later. As in Lemma 5.2 . we can compare $\widetilde{E}_{T}^{s}(v)$ with $\|v\|_{B^{s}(T)}$ if $\left\|u_{1}\right\|_{L_{T}^{\infty} H_{x}^{s}}$ is small enough.

Lemma 5.9. Let $s>\frac{1}{2}$. Then, there exists $0<\delta_{1}$ such that

$$
\frac{1}{2}\|v\|_{B^{s}(T)}^{2} \leq \widetilde{E}_{T}^{s}(v) \leq \frac{3}{2}\|v\|_{B^{s}(T)}^{2},
$$

for all $v \in B^{s}(T)$ as soon as $\left\|u_{1}\right\|_{L_{T}^{\infty} H_{x}^{s}} \leq \delta_{1}$. 
Proposition 5.10. Assume $T \in(0,1]$ and $s \geq 2$. Then, if $v$ is a solution to (5.44), we have that

$$
\begin{aligned}
& \widetilde{E}_{T}^{0}(v) \lesssim\left(1+\left\|\varphi_{1}\right\|_{H^{\frac{1}{2}+}}\right)\|\varphi\|_{L^{2}}^{2} \\
& \quad+\left(1+\left\|u_{1}\right\|_{F^{\frac{3}{4}+}(T)}\right)\left(\left\|u_{1}\right\|_{F^{2}(T)}+\left\|u_{2}\right\|_{F^{2}(T)}\right)\left(\|v\|_{B^{0}(T)}^{2}+\|v\|_{F^{0}(T)}^{2}\right)
\end{aligned}
$$

and

$$
\begin{aligned}
\widetilde{E}_{T}^{s}(v) \lesssim & \left(1+\left\|\varphi_{1}\right\|_{H^{\frac{1}{2}+}}\right)\|\varphi\|_{H^{s}}^{2}+\|v\|_{F^{0}(T)}\left\|u_{2}\right\|_{F^{s+2}(T)}\|v\|_{F^{s}(T)} \\
& +\left(1+\Gamma_{T}^{s}\left(u_{1}\right)+\Gamma_{T}^{s}\left(u_{2}\right)\right)\left(\Gamma_{T}^{s}\left(u_{1}\right)+\Gamma_{T}^{s}\left(u_{2}\right)\right) \Gamma_{T}^{s}(v)^{2}
\end{aligned}
$$

where

$$
\Gamma_{T}^{s}(u):=\max \left\{\|u\|_{F^{s}(T)},\|u\|_{B^{s}(T)}\right\} .
$$

As a corollary to Lemma 5.9 and Proposition 5.10, we deduce an a priori estimate in $\|\cdot\|_{B^{s}(T)}$ for the solutions $v$ to the difference equation (5.44).

Corollary 5.11. Assume $T \in(0,1]$. Then, there exists $0<\delta_{1} \leq 1$ such that (5.50)

$\|v\|_{B^{0}(T)}^{2} \lesssim\|\varphi\|_{L^{2}}^{2}+\left(1+\left\|u_{1}\right\|_{F^{\frac{3}{4}}+(T)}\right)\left(\left\|u_{1}\right\|_{F^{2}(T)}+\left\|u_{2}\right\|_{F^{2}(T)}\right)\left(\|v\|_{B^{0}(T)}^{2}+\|v\|_{F^{0}(T)}^{2}\right)$

and

$$
\begin{aligned}
\|v\|_{B^{s}(T)}^{2} \lesssim & \|\varphi\|_{H^{s}}^{2}+\|v\|_{F^{0}(T)}\left\|u_{2}\right\|_{F^{s+2}(T)}\|v\|_{F^{s}(T)} \\
& +\left(1+\Gamma_{T}^{s}\left(u_{1}\right)+\Gamma_{T}^{s}\left(u_{2}\right)\right)\left(\Gamma_{T}^{s}\left(u_{1}\right)+\Gamma_{T}^{s}\left(u_{2}\right)\right) \Gamma_{T}^{s}(v)^{2}
\end{aligned}
$$

for all solutions $v$ to (5.44) with $\left\|u_{1}\right\|_{L_{T}^{\infty} H_{x}^{\frac{1}{2}+}}<\delta_{1}$.

Proof of Proposition 5.10. We argue as in the proof of Proposition 5.3. First, we choose extensions $\widetilde{v}, \widetilde{u}_{1}$ and $\widetilde{u}_{2}$ of $v, u_{1}$ and $u_{2}$ over $\mathbb{R}^{2}$ satisfying

$$
\|\widetilde{v}\|_{F^{s}} \leq 2\|v\|_{F^{s}(T)} \quad \text { and } \quad\left\|\widetilde{u}_{i}\right\|_{F^{s}} \leq 2\left\|u_{i}\right\|_{F^{s}(T)}, i=1,2 .
$$

Then, for any $k \in \mathbb{Z}_{+} \cap[1,+\infty)$ and $t \in[-T, T]$, we differentiate $\widetilde{E}_{k}(v)$ with respect to $t$ and deduce using (5.44) that

$$
\frac{d}{d t} \widetilde{E}_{k}(v)=\widetilde{\mathcal{J}}_{k}(v)+\widetilde{\mathcal{J}}_{k}(v)+\widetilde{\alpha} \widetilde{\mathcal{L}}_{k}^{1}(v)+\widetilde{\alpha} \widetilde{\mathcal{N}}_{k}^{1}(v)+\widetilde{\beta}_{\mathcal{L}_{k}^{2}}^{2}(v)+\widetilde{\beta}_{\mathcal{\mathcal { N }}}^{2}(v),
$$

where

$$
\begin{gathered}
\widetilde{\mathcal{J}}_{k}(v)=4 c_{1} \int_{\mathbb{R}} P_{k} v P_{k}\left(\partial_{x} u_{1} \partial_{x}^{2} v\right) d x+4 c_{1} \int_{\mathbb{R}} P_{k} v P_{k}\left(\partial_{x} v \partial_{x}^{2} u_{2}\right) d x, \\
\widetilde{\mathcal{J}}_{k}(v)=2 c_{2} \int_{\mathbb{R}} P_{k} v P_{k} \partial_{x}\left(u_{1} \partial_{x}^{2} v\right) d x+2 c_{2} \int_{\mathbb{R}} P_{k} v P_{k} \partial_{x}\left(v \partial_{x}^{2} u_{2}\right) d x, \\
\widetilde{\mathcal{L}}_{k}^{1}(v)=\int_{\mathbb{R}} \partial_{x}^{5} u_{1} P_{k} \partial_{x}^{-1} v Q_{k} \partial_{x}^{-1} v d x+\int_{\mathbb{R}} u_{1} P_{k} \partial_{x}^{4} v Q_{k} \partial_{x}^{-1} v d x \\
+\int_{\mathbb{R}} u_{1} P_{k} \partial_{x}^{-1} v Q_{k} \partial_{x}^{4} v d x,
\end{gathered}
$$




$$
\begin{aligned}
\widetilde{\mathcal{N}}_{k}^{1}(v) & =c_{1} \int_{\mathbb{R}} \partial_{x}\left(\left(\partial_{x} u_{1}\right)^{2}\right) P_{k} \partial_{x}^{-1} v Q_{k} \partial_{x}^{-1} v d x+2 c_{1} \int_{\mathbb{R}} u_{1} P_{k} \partial_{x}^{-1}\left(\partial_{x} u_{1} \partial_{x}^{2} v\right) Q_{k} \partial_{x}^{-1} v d x \\
& +2 c_{1} \int_{\mathbb{R}} u_{1} P_{k} \partial_{x}^{-1}\left(\partial_{x} v \partial_{x}^{2} u_{2}\right) Q_{k} \partial_{x}^{-1} v d x+2 c_{1} \int_{\mathbb{R}} u_{1} P_{k} \partial_{x}^{-1} v Q_{k} \partial_{x}^{-1}\left(\partial_{x} u_{1} \partial_{x}^{2} v\right) d x \\
& +2 c_{1} \int_{\mathbb{R}} u_{1} P_{k} \partial_{x}^{-1} v Q_{k} \partial_{x}^{-1}\left(\partial_{x} v \partial_{x}^{2} u_{2}\right) d x+c_{2} \int_{\mathbb{R}} \partial_{x}\left(u_{1} \partial_{x}^{2} u_{1}\right) P_{k} \partial_{x}^{-1} v Q_{k} \partial_{x}^{-1} v d x \\
& +c_{2} \int_{\mathbb{R}} u_{1} P_{k}\left(u_{1} \partial_{x}^{2} v\right) Q_{k} \partial_{x}^{-1} v d x+c_{2} \int_{\mathbb{R}} u_{1} P_{k}\left(v \partial_{x}^{2} u_{2}\right) Q_{k} \partial_{x}^{-1} v d x \\
& +c_{2} \int_{\mathbb{R}} u_{1} P_{k} \partial_{x}^{-1} v Q_{k}\left(u_{1} \partial_{x}^{2} v\right) d x+c_{2} \int_{\mathbb{R}} u_{1} P_{k} \partial_{x}^{-1} v Q_{k}\left(v \partial_{x}^{2} u_{2}\right) d x \\
& \widetilde{\mathcal{L}}_{k}^{2}(v)=\int_{\mathbb{R}} \partial_{x}^{5} u_{1} P_{k} \partial_{x}^{-1} v P_{k} \partial_{x}^{-1} v d x+2 \int_{\mathbb{R}} u_{1} P_{k} \partial_{x}^{4} v P_{k} \partial_{x}^{-1} v d x
\end{aligned}
$$

and

$$
\begin{aligned}
\widetilde{\mathcal{N}}_{k}^{2}(v) & =c_{1} \int_{\mathbb{R}} \partial_{x}\left(\left(\partial_{x} u_{1}\right)^{2}\right) P_{k} \partial_{x}^{-1} v P_{k} \partial_{x}^{-1} v d x+4 c_{1} \int_{\mathbb{R}} u_{1} P_{k} \partial_{x}^{-1}\left(\partial_{x} u_{1} \partial_{x}^{2} v\right) P_{k} \partial_{x}^{-1} v d x \\
+ & 4 c_{1} \int_{\mathbb{R}} u_{1} P_{k} \partial_{x}^{-1}\left(\partial_{x} v \partial_{x}^{2} u_{2}\right) P_{k} \partial_{x}^{-1} v d x+c_{2} \int_{\mathbb{R}} \partial_{x}\left(u_{1} \partial_{x}^{2} u_{1}\right) P_{k} \partial_{x}^{-1} v P_{k} \partial_{x}^{-1} v d x \\
& +2 c_{2} \int_{\mathbb{R}} u_{1} P_{k}\left(u_{1} \partial_{x}^{2} v\right) P_{k} \partial_{x}^{-1} v d x+2 c_{2} \int_{\mathbb{R}} u_{1} P_{k}\left(v \partial_{x}^{2} u_{2}\right) P_{k} \partial_{x}^{-1} v d x
\end{aligned}
$$

Now, we fix $t_{k} \in[-T, T]$. Without loss of generality, we can assume that $0<t_{k} \leq$ $T$. Therefore, we obtain by integrating (5.53) between 0 and $t_{k}$ that

$$
\begin{aligned}
& \widetilde{E}_{k}(v)\left(t_{k}\right)-\widetilde{E}_{k}(v)(0) \\
& \leq\left|\int_{\left[0, t_{k}\right]}\left(\widetilde{\mathcal{J}}_{k}(v)+\widetilde{\mathcal{J}}_{k}(v)+\widetilde{\alpha} \widetilde{\mathcal{L}}_{k}^{1}(v)+\widetilde{\alpha} \widetilde{\mathcal{N}}_{k}^{1}(v)+\widetilde{\beta} \widetilde{\mathcal{L}}_{k}^{2}(v)+\widetilde{\beta} \widetilde{\mathcal{N}}_{k}^{2}(v)\right) d t\right| .
\end{aligned}
$$

Next we estimate the right-hand side of (5.55).

Estimates for the cubic terms. We deduce after some integration by parts that

$$
\widetilde{\mathcal{L}}_{k}^{1}(v)=5 \int_{\mathbb{R}} \partial_{x}^{3} u_{1} P_{k} v Q_{k} v d x-5 \int_{\mathbb{R}} \partial_{x} u_{1} P_{k} \partial_{x} v Q_{k} \partial_{x} v d x
$$

and

$$
\widetilde{\mathcal{L}}_{k}^{2}(v)=5 \int_{\mathbb{R}} \partial_{x}^{3} u_{1} P_{k} v P_{k} v d x-5 \int_{\mathbb{R}} \partial_{x} u_{1} P_{k} \partial_{x} v P_{k} \partial_{x} v d x .
$$

We choose $\widetilde{\alpha}=-\frac{2 c_{2}}{5}$ and $\widetilde{\beta}=\frac{c_{2}-4 c_{1}}{5}$. Then it follows, after performing a dyadic decomposition on $v$, that

$$
\left|\int_{\left[0, t_{k}\right]}\left(\widetilde{\mathcal{J}}_{k}(v)+\widetilde{\mathcal{J}}_{k}(v)+\widetilde{\alpha} \widetilde{\mathcal{L}}_{k}^{1}(v)+\widetilde{\beta} \widetilde{\mathcal{L}}_{k}^{2}(v)\right) d t\right| \lesssim \sum_{j=1}^{9} \widetilde{T}_{j}(k),
$$

for each $k \geq 1$, with

$$
\widetilde{T}_{1}(k)=\sum_{0 \leq k_{1} \leq k-6}\left|\int_{\mathbb{R} \times\left[0, t_{k}\right]}\left(P_{k} v P_{k}\left(P_{k_{1}} \partial_{x} u_{1} \partial_{x}^{2} v\right)+P_{k} \partial_{x} v P_{k} \partial_{x} v P_{k_{1}} \partial_{x} u_{1}\right) d x d t\right|,
$$




$$
\begin{gathered}
\widetilde{T}_{2}(k)=\sum_{0 \leq k_{1} \leq k-6}\left|\int_{\mathbb{R} \times\left[0, t_{k}\right]} P_{k} \partial_{x} v\left(\left[P_{k}, P_{k_{1}} u_{1}\right] \partial_{x}^{2} v-Q_{k} \partial_{x} v P_{k_{1}} \partial_{x} u_{1}\right) d x d t\right|, \\
\widetilde{T}_{3}(k)=\sum_{k_{1} \geq k-5, k_{2} \geq 0}\left|\int_{\mathbb{R} \times\left[0, t_{k}\right]} P_{k}^{2} v P_{k_{1}} \partial_{x} u_{1} P_{k_{2}} \partial_{x}^{2} v d t\right|, \\
\widetilde{T}_{4}(k)=\sum_{k_{1} \geq k-5, k_{2} \geq 0}\left|\int_{\mathbb{R} \times\left[0, t_{k}\right]} P_{k}^{2} \partial_{x} v P_{k_{1}} u_{1} P_{k_{2}} \partial_{x}^{2} v d t\right|, \\
\widetilde{T}_{5}(k)=\sum_{k_{1}, k_{2} \geq 0}\left|\int_{\mathbb{R} \times\left[0, t_{k}\right]} P_{k}^{2} v P_{k_{1}} \partial_{x} v P_{k_{2}} \partial_{x}^{2} u_{2} d t\right|, \\
\widetilde{T}_{6}(k)=\sum_{k_{1}, k_{2} \geq 0}\left|\int_{\mathbb{R} \times\left[0, t_{k}\right]} P_{k}^{2} \partial_{x} v P_{k_{1}} v P_{k_{2}} \partial_{x}^{2} u_{2} d t\right|, \\
\sum_{k-5 \leq k_{1} \leq k+4}\left|\int_{\mathbb{R} \times\left[0, t_{k}\right]} P_{k_{1}} \partial_{x} u_{1} P_{k} \partial_{x} v\left(Q_{k} \partial_{x} v+P_{k} \partial_{x} v\right) d x d t\right|, \\
\widetilde{T}_{8}(k)=\sum_{k_{1} \leq k-5}\left|\int_{\mathbb{R} \times\left[0, t_{k}\right]} P_{k_{1}} \partial_{x}^{3} u_{1} P_{k} v\left(Q_{k} v+P_{k} v\right) d x\right|
\end{gathered}
$$

and

$$
\widetilde{T}_{9}(k)=\sum_{k-4 \leq k_{1} \leq k+4}\left|\int_{\mathbb{R} \times\left[0, t_{k}\right]} P_{k_{1}} \partial_{x}^{3} u_{1} P_{k} v\left(Q_{k} v+P_{k} v\right) d x\right| .
$$

Clearly, Lemma 5.7 and the Cauchy-Schwarz inequality imply that

$$
\begin{aligned}
\widetilde{T}_{1}(k)+\widetilde{T}_{2}(k) & \lesssim \sum_{0 \leq k_{1} \leq k-6} 2^{k_{1} / 2}\left\|P_{k_{1}} \widetilde{u}_{1}\right\|_{F_{k_{1}}} \sum_{\left|k-k^{\prime}\right| \leq 3}\left\|P_{k^{\prime}} \widetilde{v}\right\|_{F_{k^{\prime}}}^{2} \\
& \lesssim\left\|\widetilde{u}_{1}\right\|_{F^{\frac{1}{2}+}} \sum_{\left|k-k^{\prime}\right| \leq 3}\left\|P_{k^{\prime}} \widetilde{v}\right\|_{F_{k^{\prime}}}^{2} .
\end{aligned}
$$

Similarly, we get by applying estimate (5.7) if $k_{1}=0$ and estimate (5.8) if $k_{1}>0$ that

$$
\widetilde{T}_{8}(k) \lesssim\left\|\widetilde{u}_{1}\right\|_{F^{\frac{1}{2}+}} \sum_{\left|k-k^{\prime}\right| \leq 3}\left\|P_{k^{\prime}} \widetilde{v}\right\|_{F_{k^{\prime}}}^{2}
$$

Now, estimate (5.9) leads to

$$
\widetilde{T}_{7}(k)+\widetilde{T}_{9}(k) \lesssim\left\|\widetilde{u}_{1}\right\|_{F^{\frac{5}{4}}}\left\|P_{k} \widetilde{v}\right\|_{F_{k}}^{2} .
$$

Arguing exactly as in (5.30), we get that

$$
\begin{aligned}
\widetilde{T}_{3}(k)+\widetilde{T}_{4}(k) \lesssim & \|\widetilde{v}\|_{F^{\frac{1}{2}}+} \sum_{\left|k^{\prime}-k\right| \leq 3}\left\|P_{k^{\prime}} \widetilde{u}_{1}\right\|_{F_{k^{\prime}}}\left\|P_{k^{\prime}} \widetilde{v}\right\|_{F_{k^{\prime}}} \\
& +\left\|\widetilde{u}_{1}\right\|_{F^{\frac{5}{4}}} \sum_{\left|k^{\prime}-k\right| \leq 3}\left\|P_{k^{\prime}} \widetilde{v}\right\|_{F_{k^{\prime}}}^{2} \\
& +2^{-k}\left\|P_{k} \widetilde{v}\right\|_{F_{k}} \sum_{k_{1} \geq k+5} 2^{3 k_{1} / 2}\left\|P_{k_{1}} \widetilde{u}_{1}\right\|_{F_{k_{1}}}\left\|P_{k_{1}} \widetilde{v}\right\|_{F_{k_{1}}} .
\end{aligned}
$$

This implies after taking the suprem of $t_{k}$ over $[0, T]$ and summing in $k \in \mathbb{Z}_{+} \cap$ $[1,+\infty)$ that

$$
\sum_{k \geq 1} 2^{2 k s} \sup _{t_{k} \in[0, T]}\left(\widetilde{T}_{3}(k)+\widetilde{T}_{4}(k)\right) \lesssim\left\|\widetilde{u}_{1}\right\|_{F^{\frac{5}{4}}}\|\widetilde{v}\|_{F^{s}}^{2}+\left\|\widetilde{u}_{1}\right\|_{F^{s}}\|\widetilde{v}\|_{F^{\frac{1}{2}}+}\|\widetilde{v}\|_{F^{s}}
$$


whenever $s \geq 1$ and

$$
\sum_{k \geq 1} \sup _{t_{k} \in[0, T]}\left(\widetilde{T}_{5}(k)+\widetilde{T}_{6}(k)\right) \lesssim\left\|\widetilde{u}_{1}\right\|_{F^{2}}\|\widetilde{v}\|_{F^{0}}^{2}
$$

at the $L^{2}$-level. Note that to obtain (5.62), we need to modify the first term on the right-hand side of (5.60) by putting all the derivatives on $\left\|P_{k^{\prime}} \widetilde{u}_{1}\right\|_{F_{k^{\prime}}}$.

To bound $\widetilde{T}_{5}(k)$ and $\widetilde{T}_{6}(k)$, we split the domain of summation over the $\left\{D_{j}\right\}_{j=1}^{4}$ defined in (5.34). For example, we explain how to deal with $\widetilde{T}_{6}(k)$. We have that

$$
\widetilde{T}_{6}(k)=\sum_{j=1}^{4} \sum_{\left(k_{1}, k_{2}\right) \in D_{j}}\left|\int_{\mathbb{R} \times\left[0, t_{k}\right]} P_{k}^{2} \partial_{x} v P_{k_{1}} v P_{k_{2}} \partial_{x}^{2} u_{2} d t\right| .
$$

By using estimates (5.7) when $k_{2}=0$, (5.8) when $k_{2} \geq 1$ and the Cauchy-Schwarz inequality in $k_{2}$, we deduce that

$$
\begin{aligned}
\sum_{\left(k_{1}, k_{2}\right) \in D_{1}} \mid & \int_{\mathbb{R} \times\left[0, t_{k}\right]} P_{k}^{2} \partial_{x} v P_{k_{1}} v P_{k_{2}} \partial_{x}^{2} u_{2} d t \mid \\
& \lesssim \sum_{0 \leq k_{2} \leq \max \left(k, k^{\prime}\right)-5} 2^{k_{2} / 2}\left\|P_{k_{2}} \widetilde{u}_{2}\right\|_{F_{k_{2}}} \sum_{\left|k-k^{\prime}\right| \leq 3}\left\|P_{k^{\prime}} \widetilde{v}\right\|_{F_{k^{\prime}}}^{2} \\
& \lesssim\left\|\widetilde{u}_{2}\right\|_{F^{\frac{1}{2}+}} \sum_{\left|k-k^{\prime}\right| \leq 3}\left\|P_{k^{\prime}} \widetilde{v}\right\|_{F_{k^{\prime}}}^{2} .
\end{aligned}
$$

We treat the summation over $D_{2}$ similarly. Estimate (5.7) when $k_{1}=0$, estimate (5.8) when $k_{1} \geq 1$ and the Cauchy-Schwarz inequality in $k_{1}$ imply that

$$
\begin{aligned}
\sum_{\left(k_{1}, k_{2}\right) \in D_{2}} \mid & \int_{\mathbb{R} \times\left[0, t_{k}\right]} P_{k}^{2} \partial_{x} v P_{k_{1}} v P_{k_{2}} \partial_{x}^{2} u_{2} d t \mid \\
& \lesssim \sum_{0 \leq k_{1} \leq \max \left(k, k_{2}\right)-5} 2^{-k_{1}}\left\|P_{k_{1}} \widetilde{v}\right\|_{F_{k_{1}}} \sum_{\left|k-k_{2}\right| \leq 3}\left\|P_{k} \widetilde{v}\right\|_{F_{k}} 2^{2 k_{2}}\left\|\widetilde{u}_{2}\right\|_{F_{k_{2}}} \\
& \lesssim\|\widetilde{v}\|_{F^{0}} \sum_{\left|k-k_{2}\right| \leq 3}\left\|P_{k} \widetilde{v}\right\|_{F_{k}} 2^{2 k_{2}}\left\|\widetilde{u}_{2}\right\|_{F_{k_{2}}} \cdot
\end{aligned}
$$

Estimate (5.9) gives that

$$
\sum_{\left(k_{1}, k_{2}\right) \in D_{3}}\left|\int_{\mathbb{R} \times\left[0, t_{k}\right]} P_{k}^{2} \partial_{x} v P_{k_{1}} v P_{k_{2}} \partial_{x}^{2} u_{2} d t\right| \lesssim\left\|\widetilde{u}_{2}\right\|_{F^{\frac{5}{4}}} \sum_{\left|k-k^{\prime}\right| \leq 3}\left\|P_{k^{\prime}} \widetilde{v}\right\|_{F_{k^{\prime}}}^{2}
$$

Finally, it follows from estimate (5.8) that

$$
\begin{aligned}
\sum_{\left(k_{1}, k_{2}\right) \in D_{4}} \mid \int_{\mathbb{R} \times\left[0, t_{k}\right]} & P_{k}^{2} \partial_{x} v P_{k_{1}} v P_{k_{2}} \partial_{x}^{2} u_{2} d t \mid \\
& \lesssim\left\|P_{k} \widetilde{v}\right\|_{F_{k}} \sum_{k_{2} \geq k+5} 2^{k_{2} / 2}\left\|P_{k_{2}} \widetilde{v}\right\|_{F_{k_{2}}}\left\|P_{k_{2}} \widetilde{u}_{2}\right\|_{F_{k_{2}}} .
\end{aligned}
$$


Thus, we conclude by gathering (5.63) (5.67), taking the supreme over $t_{k} \in[0, T]$, and summing in $k \in \mathbb{Z}_{+} \cap[1,+\infty)$ that

$$
\sum_{k \geq 1} 2^{2 k s} \sup _{t_{k} \in[0, T]}\left(\widetilde{T}_{5}(k)+\widetilde{T}_{6}(k)\right) \lesssim\left\|\widetilde{u}_{2}\right\|_{F^{\frac{5}{4}}}\|\widetilde{v}\|_{F^{s}}^{2}+\left\|\widetilde{u}_{2}\right\|_{F^{s+2}}\|\widetilde{v}\|_{F^{0}}\|\widetilde{v}\|_{F^{s}}
$$

whenever $s \geq 1$ and

$$
\sum_{k \geq 1} \sup _{t_{k} \in[0, T]}\left(\widetilde{T}_{5}(k)+\widetilde{T}_{6}(k)\right) \lesssim\left\|\widetilde{u}_{2}\right\|_{F^{2}}\|\widetilde{v}\|_{F^{0}}^{2}
$$

at the $L^{2}$-level.

Therefore, we deduce by gathering (5.57) $-(5.61)$ and (5.68) that

$$
\begin{aligned}
& \sum_{k \geq 1} 2^{2 k s} \sup _{t_{k} \in[0, T]}\left|\int_{\left[0, t_{k}\right]}\left(\widetilde{\mathcal{J}}_{k}(v)+\widetilde{\mathcal{J}}_{k}(v)+\widetilde{\alpha} \widetilde{\mathcal{L}}_{k}^{1}(v)+\widetilde{\beta} \widetilde{\mathcal{L}}_{k}^{2}(v)\right) d t\right| \\
& \lesssim\left(\left\|u_{1}\right\|_{F^{\frac{5}{4}(T)}}+\left\|u_{2}\right\|_{F^{\frac{5}{4}(T)}}\right)\|v\|_{F^{s}(T)}^{2}+\left\|u_{1}\right\|_{F^{s}(T)}\|v\|_{F^{\frac{1}{2}+}(T)}\|v\|_{F^{s}(T)} \\
& \quad+\left\|u_{2}\right\|_{F^{s+2}(T)}\|v\|_{F^{0}(T)}\|v\|_{F^{s}(T)}
\end{aligned}
$$

if $s \geq 1$, whereas

$$
\begin{aligned}
\sum_{k \geq 1} \sup _{t_{k} \in[0, T]} \mid \int_{\left[0, t_{k}\right]}\left(\widetilde{\mathcal{J}}_{k}(v)+\right. & \left.\widetilde{\mathcal{J}}_{k}(v)+\widetilde{\alpha} \widetilde{\mathcal{L}}_{k}^{1}(v)+\widetilde{\beta} \widetilde{\mathcal{L}}_{k}^{2}(v)\right) d t \mid \\
& \lesssim\left(\left\|u_{1}\right\|_{F^{2}(T)}+\left\|u_{2}\right\|_{F^{2}(T)}\right)\|v\|_{F^{0}(T)}^{2}
\end{aligned}
$$

at the $L^{2}$ level.

Estimates for the fourth-order terms. We estimate the fourth-order term corresponding to $\widetilde{\mathcal{N}}_{k}^{2}(v)$. After some integration by parts in (5.54), we get that

$$
\left|\int_{\left[0, t_{k}\right]} \tilde{\mathcal{N}}_{k}^{2}(v) d t\right| \lesssim \sum_{i=1}^{5} \widetilde{X}_{i}(k),
$$

for each $k \geq 1$, with

$$
\begin{gathered}
\tilde{X}_{1}(k)=\left|\int_{\mathbb{R} \times\left[0, t_{k}\right]}\left(\partial_{x} u_{1}\right)^{2} P_{k} \partial_{x}^{-1} v P_{k} v d x d t\right|+\left|\int_{\mathbb{R} \times\left[0, t_{k}\right]} u_{1} \partial_{x} u_{1} P_{k} v P_{k} v d x d t\right| \\
+\left|\int_{\mathbb{R} \times\left[0, t_{k}\right]} u_{1} \partial_{x} u_{1} P_{k} \partial_{x} v P_{k} \partial_{x}^{-1} v d x d t\right|, \\
\widetilde{X}_{2}(k)=\left|\int_{\mathbb{R} \times\left[0, t_{k}\right]} u_{1} P_{k} \partial_{x}^{-1}\left(\partial_{x} v \partial_{x}^{2} u_{2}\right) P_{k} \partial_{x}^{-1} v d x d t\right|, \\
\widetilde{X}_{3}(k)=\left|\int_{\mathbb{R} \times\left[0, t_{k}\right]} u_{1} P_{k} \partial_{x}^{-1}\left(\partial_{x} u_{1} \partial_{x}^{2} v\right) P_{k} \partial_{x}^{-1} v d x d t\right|, \\
\widetilde{X}_{4}(k)=\left|\int_{\mathbb{R} \times\left[0, t_{k}\right]} u_{1} P_{k}\left(v \partial_{x}^{2} u_{2}\right) P_{k} \partial_{x}^{-1} v d x d t\right|
\end{gathered}
$$

and

$$
\widetilde{X}_{5}(k)=\left|\int_{\mathbb{R} \times\left[0, t_{k}\right]} u_{1} P_{k}\left(u_{1} \partial_{x}^{2} v\right) P_{k} \partial_{x}^{-1} v d x d t\right|
$$


We use the Strichartz estimate (2.47) with $\alpha=2$, estimate (2.10) and Hölder's inequality to deduce that

$$
\begin{aligned}
& \sum_{k \geq 1} 2^{2 k s} \sup _{t_{k} \in[0, T]} \tilde{X}_{1}(k) \\
& \quad \lesssim\left(\left\|u_{1}\right\|_{L_{T}^{2} L_{x}^{\infty}}+\left\|\partial_{x} u_{1}\right\|_{L_{T}^{2} L_{x}^{\infty}}\right)\left\|\partial_{x} u_{1}\right\|_{L_{T}^{2} L_{x}^{\infty}} \sum_{k \geq 1} 2^{2 k s}\left\|P_{k} v\right\|_{L_{T}^{\infty} L_{x}^{2}}^{2} \\
& \quad \lesssim\left(\left\|u_{1}\right\|_{F^{\frac{1}{2}+}(T)}+\left\|u_{1}\right\|_{F^{\frac{3}{4}+}(T)}\right)\left\|u_{1}\right\|_{F^{\frac{3}{4}+}(T)}\|v\|_{B^{s}(T)}^{2},
\end{aligned}
$$

for any $s \geq 0$.

To handle $\widetilde{X}_{2}(k)$, we perform the following decomposition:

$$
\begin{aligned}
\tilde{X}_{2}(k) & \lesssim \sum_{j=1}^{4} \sum_{\left(k_{1}, k_{2}, k_{3}\right) \in F_{j}}\left|\int_{\mathbb{R} \times\left[0, t_{k}\right]} P_{k_{1}} u_{1} P_{k} \partial_{x}^{-1}\left(P_{k_{2}} \partial_{x} v \partial_{x} P_{k_{3}} \partial_{x}^{2} u_{2}\right) P_{k} \partial_{x}^{-1} v d x d t\right| \\
& =: \sum_{j=0}^{4} \tilde{X}_{2, j}(k),
\end{aligned}
$$

where

$$
\begin{aligned}
& F_{1}=\left\{\left(k_{1}, k_{2}, k_{3}\right) \in \mathbb{Z}_{+}^{3}: 0 \leq k_{1} \leq k+3,\left|k-k_{3}\right| \leq 3,0 \leq k_{2} \leq \max \left(k, k_{3}\right)-5\right\} \text {, } \\
& F_{2}=\left\{\left(k_{1}, k_{2}, k_{3}\right) \in \mathbb{Z}_{+}^{3}: 0 \leq k_{1} \leq k+3,\left|k-k_{2}\right| \leq 3,0 \leq k_{3} \leq \max \left(k, k_{2}\right)-5\right\} \text {, } \\
& F_{3}=\left\{\left(k_{1}, k_{2}, k_{3}\right) \in \mathbb{Z}_{+}^{3}: 0 \leq k_{1} \leq k+3,\left|k-k_{2}\right| \leq 8,\left|k-k_{3}\right| \leq 8\right\} \text {, } \\
& F_{4}=\left\{\left(k_{1}, k_{2}, k_{3}\right) \in \mathbb{Z}_{+}^{3}: 0 \leq k_{1} \leq k+3,\left|k_{2}-k_{3}\right| \leq 3,1 \leq k \leq \max \left(k_{2}, k_{3}\right)-8\right\} \text {. }
\end{aligned}
$$

By applying Hölder's inequality, we can bound $\widetilde{X}_{2,1}(k)$ by

$$
\begin{aligned}
\sum_{0 \leq k_{1} \leq k+3}\left\|P_{k_{1}} u_{1}\right\|_{L_{T, x}^{\infty}} \sum_{0 \leq k_{2} \leq \max \left(k, k_{3}\right)-5} 2^{k_{2}}\left\|P_{k_{2}} v\right\|_{L_{T}^{2} L_{x}^{\infty}} & \times \sum_{\left|k-k_{3}\right| \leq 3}\left\|P_{k_{3}} u_{2}\right\|_{L_{T}^{\infty} L_{x}^{2}}\left\|P_{k} v\right\|_{L_{T}^{\infty} L_{x}^{2}},
\end{aligned}
$$

which implies after using the Sobolev embedding, the Cauchy-Schwarz inequality and estimate (2.48) that

$$
\sum_{k \geq 1} 2^{2 k s} \sup _{t_{k} \in[0, T]} \widetilde{X}_{2,1}(k) \lesssim\left\|u_{1}\right\|_{F^{\frac{1}{2}+}(T)}\|v\|_{F^{\frac{3}{4}+}(T)}\left\|u_{2}\right\|_{B^{s}(T)}\|v\|_{B^{s}(T)},
$$

for any $s \geq 0$. On the other hand, by putting the $L_{T}^{\infty} L_{x}^{2}$ norm on $P_{k_{2}} v$ and the $L_{T}^{2} L_{x}^{\infty}$ norm on $P_{k_{3}} u_{2}$ in (5.75), we get that

$$
\sum_{k \geq 1} \sup _{t_{k} \in[0, T]} \widetilde{X}_{2,1}(k) \lesssim\left\|u_{1}\right\|_{F^{\frac{1}{2}+}(T)}\left\|u_{2}\right\|_{F^{\frac{3}{4}+}(T)}\|v\|_{B^{0}(T)}^{2}
$$

at the $L^{2}$ level. By using similar arguments, we get that

$$
\sum_{k \geq 1} 2^{2 k s} \sup _{t_{k} \in[0, T]}\left(\widetilde{X}_{2,2}(k)+\tilde{X}_{2,3}(k)\right) \lesssim\left\|u_{1}\right\|_{F^{\frac{1}{2}+}(T)}\left\|u_{2}\right\|_{F^{\frac{3}{4}+}(T)}\|v\|_{B^{s}(T)}^{2}
$$

for any $s \geq 0$. Finally, we use estimate (5.20) to bound $\widetilde{X}_{2,4}(k)$ by

$\sum_{0 \leq k_{1} \leq k+3}\left\|P_{k_{1}} \widetilde{u}_{1}\right\|_{F_{k_{1}}} 2^{-\frac{3 k}{2}}\left\|P_{k} \widetilde{v}\right\|_{F_{k}} \sum_{\max \left(k_{2}, k_{3}\right) \geq k+8} \sum_{\left|k_{2}-k_{3}\right| \leq 3}\left\|P_{k_{2}} \widetilde{v}\right\|_{F_{k_{2}}} 2^{2 k_{3}}\left\|P_{k_{3}} \widetilde{u}_{2}\right\|_{F_{k_{3}}}$, 
which implies after summing over $k \in \mathbb{Z}_{+} \cap[1,+\infty)$ that

$$
\sum_{k \geq 1} 2^{2 k s} \sup _{t_{k} \in[0, T]} \widetilde{X}_{2,4}(k) \lesssim\left\|\widetilde{u}_{1}\right\|_{F^{0+}}\left\|\widetilde{u}_{2}\right\|_{F^{2}}\|\widetilde{v}\|_{F^{s}}^{2},
$$

for all $s \geq 0$. Therefore, we conclude by gathering estimates (5.52) and (5.74) $-(5.79)$ that

$$
\begin{aligned}
\sum_{k \geq 1} 2^{2 k s} \sup _{t_{k} \in[0, T]} \widetilde{X}_{2}(k) \lesssim & \left\|u_{1}\right\|_{F^{\frac{1}{2}+}(T)}\left\|u_{2}\right\|_{F^{2}(T)}\left(\|v\|_{B^{s}(T)}^{2}+\|v\|_{F^{s}(T)}^{2}\right) \\
& +\left\|u_{1}\right\|_{F^{\frac{1}{2}+}(T)}\|v\|_{F^{\frac{3}{4}+}(T)}\left\|u_{2}\right\|_{B^{s}(T)}\|v\|_{B^{s}(T)}
\end{aligned}
$$

for any $s \geq 0$, and

$$
\sum_{k \geq 1} \sup _{t_{k} \in[0, T]} \widetilde{X}_{2}(k) \lesssim\left\|u_{1}\right\|_{F^{\frac{1}{2}+}(T)}\left\|u_{2}\right\|_{F^{2}(T)}\left(\|v\|_{B^{0}(T)}^{2}+\|v\|_{F^{0}(T)}^{2}\right)
$$

at the $L^{2}$ level.

By using the same arguments as for $\widetilde{X}_{2}(k)$, we have that

$$
\begin{aligned}
\sum_{k \geq 1} 2^{2 k s} \sup _{t_{k} \in[0, T]} \widetilde{X}_{3}(k) \lesssim & \left\|u_{1}\right\|_{F^{\frac{1}{2}+}(T)}\left\|u_{1}\right\|_{F^{2}(T)}\left(\|v\|_{B^{s}(T)}^{2}+\|v\|_{F^{s}(T)}^{2}\right) \\
& +\left\|u_{1}\right\|_{F^{\frac{1}{2}+}(T)}\|v\|_{F^{\frac{3}{4}+}(T)}\left\|u_{1}\right\|_{B^{s}(T)}\|v\|_{B^{s}(T)}
\end{aligned}
$$

for any $s \geq 0$, and

$$
\sum_{k \geq 1} \sup _{t_{k} \in[0, T]} \tilde{X}_{3}(k) \lesssim\left\|u_{1}\right\|_{F^{\frac{1}{2}+}(T)}\left\|u_{1}\right\|_{F^{2}(T)}\left(\|v\|_{B^{0}(T)}^{2}+\|v\|_{F^{0}(T)}^{2}\right)
$$

at the $L^{2}$ level.

To deal with $\widetilde{X}_{4}(k)$ at the $L^{2}$ level, we observe after integrating by parts that

$$
\begin{aligned}
\widetilde{X}_{4}(k) \leq & \left|\int_{\mathbb{R} \times\left[0, t_{k}\right]} \partial_{x} u_{1} P_{k}\left(v \partial_{x} u_{2}\right) P_{k} \partial_{x}^{-1} v d x d t\right| \\
& +\left|\int_{\mathbb{R} \times\left[0, t_{k}\right]} u_{1} P_{k}\left(\partial_{x} v \partial_{x} u_{2}\right) P_{k} \partial_{x}^{-1} v d x d t\right| \\
& +\left|\int_{\mathbb{R} \times\left[0, t_{k}\right]} u_{1} P_{k}\left(v \partial_{x} u_{2}\right) P_{k} v d x d t\right| \\
= & : \sum_{j=1}^{3} \widetilde{X}_{4, j}(k) .
\end{aligned}
$$

Arguing exactly as for $X_{2}(k)$ in (5.35), we deduce that

$$
\begin{aligned}
& \sum_{k \geq 1} 2^{2 k s} \sup _{t_{k} \in[0, T]}\left(\widetilde{X}_{4,1}(k)+\tilde{X}_{4,2}(k)\right) \\
& \lesssim\left\|u_{1}\right\|_{F^{\frac{3}{4}+}(T)}\left\|u_{2}\right\|_{F^{2}(T)}\|v\|_{B^{s}(T)}^{2}+\left\|u_{1}\right\|_{F^{\frac{3}{4}+}(T)}\|v\|_{F^{\frac{3}{4}+}(T)}\left\|u_{2}\right\|_{B^{s}(T)}\|v\|_{B^{s}(T)},
\end{aligned}
$$

for all $s \geq 0$, and

$$
\sum_{k \geq 1} \sup _{t_{k} \in[0, T]} \widetilde{X}_{4}(k) \lesssim\left\|u_{1}\right\|_{F^{\frac{3}{4}+}(T)}\left\|u_{2}\right\|_{F^{2}(T)}\|v\|_{B^{0}(T)}^{2}
$$


at the $L^{2}$ level. To estimate $\widetilde{X}_{4,3}(k)$ at the $H^{s}$-level, we use the same decomposition as for $X_{4}(k)$ in (5.37). It follows that

$$
\begin{aligned}
\sum_{k \geq 1} 2^{2 k s} \sup _{t_{k} \in[0, T]} \widetilde{X}_{4,3}(k) \lesssim & \left\|u_{1}\right\|_{F^{\frac{1}{2}+}(T)}\left\|u_{2}\right\|_{F^{\frac{3}{4}+}(T)}\|v\|_{B^{s}(T)}^{2} \\
& +\left\|u_{2}\right\|_{F^{\frac{3}{4}+}(T)}\|v\|_{F^{\frac{1}{2}+}(T)}\left\|u_{1}\right\|_{B^{s}(T)}\|v\|_{B^{s}(T)} \\
& +\left\|u_{1}\right\|_{F^{\frac{1}{2}+}(T)}\|v\|_{F^{\frac{1}{2}+}(T)}\left\|u_{2}\right\|_{F^{s}(T)}\|v\|_{F^{s}(T)}
\end{aligned}
$$

which implies, together with (5.84) and (5.85), that

$$
\begin{aligned}
& \sum_{k \geq 1} 2^{2 k s} \sup _{t_{k} \in[0, T]} \tilde{X}_{4}(k) \\
& \lesssim\left\|u_{1}\right\|_{F^{\frac{3}{4}+}(T)}\left\|u_{2}\right\|_{F^{2}(T)}\|v\|_{B^{s}(T)}^{2}+\left\|u_{1}\right\|_{F^{\frac{1}{2}+}(T)}\|v\|_{F^{\frac{1}{2}+}(T)}\left\|u_{2}\right\|_{F^{s}(T)}\|v\|_{F^{s}(T)} \\
& \quad+\left(\left\|u_{1}\right\|_{F^{\frac{3}{4}+}(T)}+\left\|u_{2}\right\|_{F^{\frac{3}{4}+}(T)}\right)\|v\|_{F^{\frac{3}{4}+}(T)}\left(\left\|u_{1}\right\|_{B^{s}(T)}+\left\|u_{2}\right\|_{B^{s}(T)}\right)\|v\|_{B^{s}(T)},
\end{aligned}
$$

for all $s \geq 0$.

Finally, we treat the term $\widetilde{X}_{5}(k)$. After integrating by parts, we obtain that

$$
\begin{aligned}
\widetilde{X}_{5}(k) \leq & \left|\int_{\mathbb{R} \times\left[0, t_{k}\right]} \partial_{x} u_{1} P_{k}\left(u_{1} \partial_{x} v\right) P_{k} \partial_{x}^{-1} v d x d t\right| \\
& +\left|\int_{\mathbb{R} \times\left[0, t_{k}\right]} u_{1} P_{k}\left(\partial_{x} u_{1} \partial_{x} v\right) P_{k} \partial_{x}^{-1} v d x d t\right| \\
& +\left|\int_{\mathbb{R} \times\left[0, t_{k}\right]} u_{1} P_{k}\left(u_{1} \partial_{x} v\right) P_{k} v d x d t\right| \\
:= & \sum_{j=1}^{3} \widetilde{X}_{5, j}(k) .
\end{aligned}
$$

By using the same arguments as above and arguing exactly as for $X_{2}(k)$ in (5.35), we deduce that

$$
\begin{aligned}
& \sum_{k \geq 1} 2^{2 k s} \sup _{t_{k} \in[0, T]}\left(\widetilde{X}_{5,1}(k)+\widetilde{X}_{5,2}(k)\right) \\
& \lesssim\left\|u_{1}\right\|_{F^{\frac{3}{4}+}(T)}\left\|u_{1}\right\|_{F^{2}(T)}\|v\|_{B^{s}(T)}^{2}+\left\|u_{1}\right\|_{F^{\frac{3}{4}+}(T)}\|v\|_{F^{\frac{3}{4}+}(T)}\left\|u_{1}\right\|_{B^{s}(T)}\|v\|_{B^{s}(T)},
\end{aligned}
$$

for all $s \geq 0$, and

$$
\sum_{k \geq 1} \sup _{t_{k} \in[0, T]}\left(\widetilde{X}_{5,1}(k)+\widetilde{X}_{5,2}(k)\right) \lesssim\left\|u_{1}\right\|_{F^{\frac{3}{4}+}(T)}\left\|u_{1}\right\|_{F^{2}(T)}\|v\|_{B^{0}(T)}^{2}
$$

at the $L^{2}$ level. To handle $\widetilde{X}_{5,3}(k)$, we perform the same decomposition as for $X^{4}(k)$ in (5.37). It follows that

$$
\begin{aligned}
\sum_{k \geq 1} 2^{2 k s} \sup _{t_{k} \in[0, T]} \widetilde{X}_{5,3}(k) \lesssim & \left\|u_{1}\right\|_{F^{\frac{1}{2}+}(T)}\|v\|_{F^{\frac{3}{4}+}(T)}\left\|u_{1}\right\|_{B^{s}(T)}\|v\|_{B^{s}(T)} \\
& +\left\|u_{1}\right\|_{F^{\frac{1}{2}+}(T)}\left\|u_{1}\right\|_{F^{\frac{3}{4}+}(T)}\left(\|v\|_{F^{s}(T)}^{2}+\|v\|_{B^{s}(T)}^{2}\right)
\end{aligned}
$$


for any $s \geq 0$, and

$$
\sum_{k \geq 1} \sup _{t_{k} \in[0, T]} \widetilde{X}_{5,3}(k) \lesssim\left\|u_{1}\right\|_{F^{\frac{1}{2}+}(T)}\left\|u_{1}\right\|_{F^{\frac{3}{4}+}(T)}\left(\|v\|_{F^{0}(T)}^{2}+\|v\|_{B^{0}(T)}^{2}\right)
$$

at the $L^{2}$ level. Thus, we deduce from (5.89) and (5.91) that

$$
\begin{aligned}
\sum_{k \geq 1} 2^{2 k s} \sup _{t_{k} \in[0, T]} \widetilde{X}_{5}(k) \lesssim & \left\|u_{1}\right\|_{F^{\frac{3}{4}+}(T)}\|v\|_{F^{\frac{3}{4}+}(T)}\left\|u_{1}\right\|_{B^{s}(T)}\|v\|_{B^{s}(T)} \\
& +\left\|u_{1}\right\|_{F^{\frac{3}{4}+}(T)}\left\|u_{1}\right\|_{F^{2}(T)}\left(\|v\|_{F^{s}(T)}^{2}+\|v\|_{B^{s}(T)}^{2}\right),
\end{aligned}
$$

for any $s \geq 0$, and from (5.90) and (5.92) that

$$
\sum_{k \geq 1} \sup _{t_{k} \in[0, T]} \widetilde{X}_{5}(k) \lesssim\left\|u_{1}\right\|_{F^{\frac{3}{4}+}(T)}\left\|u_{1}\right\|_{F^{2}(T)}\left(\|v\|_{F^{0}(T)}^{2}+\|v\|_{B^{0}(T)}^{2}\right)
$$

at the $L^{2}$ level.

Therefore, we deduce from (5.72), (5.73), (5.81), (5.83), (5.86) and (5.94) that

$$
\begin{aligned}
\sum_{k \geq 1} \sup _{t_{k} \in[0, T]} \mid & \int_{\left[0, t_{k}\right]} \tilde{\mathcal{N}}_{k}^{2}(v) d t \mid \\
& \lesssim\left\|u_{1}\right\|_{F^{\frac{3}{4}+}(T)}\left(\left\|u_{1}\right\|_{F^{2}(T)}+\left\|u_{2}\right\|_{F^{2}(T)}\right)\left(\|v\|_{F^{0}(T)}^{2}+\|v\|_{B^{0}(T)}^{2}\right),
\end{aligned}
$$

which together with (5.55) and (5.71) implies estimate (5.48), since the bound for the term corresponding to $\tilde{\mathcal{N}}_{k}^{1}(v)$ would be similar.

Similarly, we deduce from (5.72), (5.73), (5.80), (5.82), (5.87) and (5.93) that

$$
\begin{aligned}
& \sum_{k \geq 1} 2^{2 k s} \sup _{t_{k} \in[0, T]}\left|\int_{\left[0, t_{k}\right]} \tilde{\mathcal{N}}_{k}^{2}(v) d t\right| \\
& \lesssim\left(\left\|u_{1}\right\|_{F^{\frac{3}{4}+}(T)}+\left\|u_{2}\right\|_{F^{\frac{3}{4}+}(T)}\right)\|v\|_{F^{\frac{3}{4}+}(T)}\left(\left\|u_{1}\right\|_{B^{s}(T)}+\left\|u_{2}\right\|_{B^{s}(T)}\right)\|v\|_{B^{s}(T)} \\
& \quad+\left\|u_{1}\right\|_{F^{\frac{3}{4}+}(T)}\left(\left\|u_{1}\right\|_{F^{2}(T)}+\left\|u_{2}\right\|_{F^{2}(T)}\right)\left(\|v\|_{F^{s}(T)}^{2}+\|v\|_{B^{s}(T)}^{2}\right) \\
& \quad+\left\|u_{1}\right\|_{F^{\frac{1}{2}+}(T)}\|v\|_{F^{\frac{1}{2}+}(T)}\left\|u_{2}\right\|_{F^{s}(T)}\|v\|_{F^{s}(T)},
\end{aligned}
$$

which together with (5.55) and (5.70) implies estimate (5.49).

This concludes the proof of Proposition 5.10 .

\section{Proof of Theorem 1.1}

We recall, for the sake of simplicity, that we are proving Theorem 1.1 in the case $c_{3}=0$. The starting point is a well-posedness result for smooth solutions which follows from Theorem 3.1 in 33 .

Theorem 6.1. For all $u_{0} \in H^{\infty}(\mathbb{R})$, there exist a positive time $T$ and a unique solution $u \in C\left([-T, T] ; H^{\infty}(\mathbb{R})\right)$ to the initial value problem (1.1). Moreover $T=$ $T\left(\left\|u_{0}\right\|_{H^{4}}\right)$ can be chosen as a nonincreasing function of its argument.

6.1. A priori estimates for smooth solutions. The main result of this subsection reads as follows.

Proposition 6.2. Assume $s \geq \frac{5}{4}$. For any $M>0$, there exists a positive time $T=T(M)$ such that for any initial data $u_{0} \in H^{\infty}(\mathbb{R})$ satisfying $\left\|u_{0}\right\|_{H^{s}} \leq M$, the solution u obtained in Theorem 6.1 is defined on $[-T, T]$ and satisfies

$$
u \in C\left([-T, T] ; H^{\infty}(\mathbb{R})\right) \quad \text { and } \quad\|u\|_{L_{T}^{\infty} H_{x}^{s}} \lesssim\left\|u_{0}\right\|_{H^{s}} .
$$


The following technical lemma will be needed in the proof of Proposition 6.2.

Lemma 6.3. Assume $s \in \mathbb{R}_{+}, T>0$ and $u \in C\left([-T, T] ; H^{\infty}(\mathbb{R})\right)$. We define

$$
\Lambda_{T^{\prime}}^{s}(u):=\max \left\{\|u\|_{B^{s}\left(T^{\prime}\right)},\left\|\partial_{x}\left(u \partial_{x}^{2} u\right)\right\|_{N^{s}\left(T^{\prime}\right)},\left\|\partial_{x} u \partial_{x}^{2} u\right\|_{N^{s}\left(T^{\prime}\right)}\right\},
$$

for any $0 \leq T^{\prime} \leq T$. Then : $T^{\prime} \mapsto \Lambda_{T^{\prime}}^{s}(u)$ is nondecreasing and continuous on $[0, T)$. Moreover

$$
\lim _{T^{\prime} \rightarrow 0} \Lambda_{T^{\prime}}^{s}(u) \lesssim\|u(0)\|_{H^{s}} .
$$

Proof. It is clear from the definition of $B^{s}\left(T^{\prime}\right)$ and the fact that $u \in$ $C\left([-T, T] ; H^{\infty}(\mathbb{R})\right)$ that : $T^{\prime} \mapsto\|u\|_{B^{s}\left(T^{\prime}\right)}$ is nondecreasing and continuous on $[0, T]$ and that

$$
\lim _{T^{\prime} \rightarrow 0}\|u\|_{B^{s}\left(T^{\prime}\right)} \lesssim\|u(0)\|_{H^{s}} .
$$

In order to deal with the other components of $\Lambda_{T^{\prime}}^{s}(u)$ in (6.2), it suffices to prove that given $f \in C\left([-T, T] ; H^{\infty}(\mathbb{R})\right)$,

$$
: T^{\prime} \in[0, T) \mapsto\|f\|_{N^{s}\left(T^{\prime}\right)} \text { is continuous and nondecreasing }
$$

and

$$
\lim _{T^{\prime} \rightarrow 0}\|f\|_{N^{s}\left(T^{\prime}\right)}=0 .
$$

It is clear from the definition of $N^{s}$ that

$$
\|\widetilde{f}\|_{N^{s}} \lesssim\|\widetilde{f}\|_{L_{t}^{2} H_{x}^{s}}
$$

for any $\tilde{f} \in L_{t}^{2} H_{x}^{s}$. Then, we deduce by applying estimate (6.7) to $\tilde{f}(x, t)=$ $\chi_{\left[-T^{\prime}, T^{\prime}\right]}(t) f(x, t)$ that

$$
\|f\|_{N^{s}\left(T^{\prime}\right)} \leq\|\widetilde{f}\|_{N^{s}} \lesssim\|\widetilde{f}\|_{L_{t}^{2} H_{x}^{s}} \lesssim\left(T^{\prime}\right)^{1 / 2}\|f\|_{L_{T}^{\infty} H_{x}^{s} \underset{T^{\prime} \rightarrow 0}{\longrightarrow} 0}^{\longrightarrow}
$$

which gives (6.6).

Now, we turn to the proof of (6.5). The fact that : $T^{\prime} \in[0, T) \mapsto\|f\|_{N^{s}\left(T^{\prime}\right)}$ is a nondecreasing function follows directly from the definition of $N^{s}\left(T^{\prime}\right)$. To prove the continuity of : $T^{\prime} \in(0, T) \mapsto\|f\|_{N^{s}\left(T^{\prime}\right)}$ at some fixed time $T_{0}^{\prime} \in(0, T)$, we introduce the scaling operator $D_{r}(f)(x, t):=f\left(x / r^{\frac{1}{5}}, t / r\right)$, for $r$ close enough to 1 . Hence, we have from (6.8) and the triangle inequality that

$$
\begin{aligned}
& \left|\|f\|_{N^{s}\left(T^{\prime}\right)}-\left\|D_{T^{\prime} / T_{0}^{\prime}}(f)\right\|_{N^{s}\left(T^{\prime}\right)}\right| \leq\left\|f-D_{T^{\prime} / T_{0}^{\prime}}(f)\right\|_{N^{s}\left(T^{\prime}\right)} \\
& \lesssim\left(T^{\prime}\right)^{\frac{1}{2}}\left\|f-D_{T^{\prime} / T_{0}^{\prime}}(f)\right\|_{L_{T}^{\infty} H_{x}^{s}}^{\underset{T^{\prime} \rightarrow T_{0}^{\prime}}{\rightarrow} 0,}
\end{aligned}
$$

since $f \in C\left([-T, T] ; H^{\infty}(\mathbb{R})\right)$. Then, it remains to show that

$$
\lim _{r \rightarrow 1}\left\|D_{r}(f)\right\|_{N^{s}\left(r T_{0}^{\prime}\right)}=\|f\|_{N^{s}\left(T_{0}^{\prime}\right)}
$$

to conclude the proof of (6.5). We observe that (6.10) would follow from the inequalities

$$
\|f\|_{N^{s}\left(T_{0}^{\prime}\right)} \leq \liminf _{r \rightarrow 1}\left\|D_{r}(f)\right\|_{N^{s}\left(r T_{0}^{\prime}\right)}
$$

and

$$
\limsup _{r \rightarrow 1}\left\|D_{r}(f)\right\|_{N^{s}\left(r T_{0}^{\prime}\right)} \leq\|f\|_{N^{s}\left(T_{0}^{\prime}\right)} .
$$


First, we begin with the proof of (6.11). Let $\epsilon$ be an arbitrarily small positive number. For $r$ close to 1 , we choose an extension $\widetilde{f}_{r}$ of $D_{r}(f)$ outside of $\left[-r T_{0}^{\prime}, r T_{0}^{\prime}\right]$ satisfying

$$
\tilde{f}_{\left.r\right|_{\left[-r T_{0}^{\prime}, r T_{0}^{\prime}\right]}}=D_{r}(f) \quad \text { and } \quad\left\|\tilde{f}_{r}\right\|_{N^{s}} \leq\left\|D_{r}(f)\right\|_{N^{s}\left(r T_{0}^{\prime}\right)}+\epsilon .
$$

Note that since $f \in C\left([-T, T] ; H^{\infty}(\mathbb{R})\right)$, we have $\left\|D_{r}(f)\right\|_{N^{s}\left(r T_{0}^{\prime}\right)} \leq M$, where $M$ is a positive constant independent of $r$. We also observe that $D_{1 / r}\left(\widetilde{f}_{r}\right)$ is an extension of $f$ outside of $\left[-T_{0}^{\prime}, T_{0}^{\prime}\right]$, so that

$$
\|f\|_{N^{s}\left(T_{0}^{\prime}\right)} \leq\left\|D_{1 / r}\left(\widetilde{f}_{r}\right)\right\|_{N^{s}} .
$$

Moreover, we will prove that

$$
\left\|D_{1 / r}\left(\tilde{f}_{r}\right)\right\|_{N^{s}} \leq \psi(r)\left\|\widetilde{f}_{r}\right\|_{N^{s}},
$$

where $\psi$ is a continuous function defined in a neighborhood of 1 and satisfying $\lim _{r \rightarrow 1} \psi(r)=1$. Then estimate (6.11) would be deduced by gathering estimates (6.13), (6.14) and (6.15).

To prove estimate (6.15), we first fix $k \in \mathbb{Z}_{+}$. Then, by definition,

$$
\left\|P_{k} D_{1 / r}\left(\tilde{f}_{r}\right)\right\|_{N_{k}}=\sup _{\widetilde{t} \in \mathbb{R}}\left\|\left(\tau-w(\xi)+i 2^{2 k}\right)^{-1} \mathcal{F}\left[\eta_{0}\left(2^{2 k}(\cdot-\widetilde{t})\right) P_{k} D_{1 / r}\left(\tilde{f}_{r}\right)\right]\right\|_{X_{k}}
$$

We observe that

$$
\eta_{0}\left(2^{2 k}(\cdot-\widetilde{t})\right) D_{1 / r}\left(\widetilde{f}_{r}\right)=D_{1 / r}\left(\eta_{0}^{r}\left(2^{2 k}(\cdot-r \widetilde{t})\right) \tilde{f}_{r}\right),
$$

where $\eta_{0}^{r}(t)=\eta_{0}\left(r^{-1} t\right)$. Hence,

$$
\mathcal{F}\left[\eta_{0}\left(2^{2 k}(\cdot-\widetilde{t})\right) P_{k} D_{1 / r}\left(\tilde{f}_{r}\right)\right](\xi, \tau)=r^{-\frac{6}{5}} \eta_{k}(\xi) \mathcal{F}\left[\eta_{0}^{r}\left(2^{2 k}(\cdot-r \widetilde{t})\right) \tilde{f}_{r}\right]\left(\xi / r^{\frac{1}{5}}, \tau / r\right),
$$

so we deduce from the definition of $X_{k}$ in (2.4) that the right-hand side of (6.16) is equal to

$$
r^{-3 / 5} \sup _{\widetilde{t} \in \mathbb{R}} \sum_{j \geq 0} 2^{j / 2}\left\|\frac{\eta_{j}(r(\tau-w(\xi)))}{\left|r(\tau-w(\xi))+i 2^{2 k}\right|} \eta_{k}\left(r^{\frac{1}{5}} \xi\right) \mathcal{F}\left[\eta_{0}^{r}\left(2^{2 k}(\cdot-\widetilde{t})\right) \tilde{f}_{r}\right]\right\|_{L_{\xi, \tau}^{2}}
$$

Moreover, we use that

$$
\left|\frac{a^{2}+2^{4 k}}{r^{2} a^{2}+2^{4 k}}-1\right|=\left|1-r^{2}\right| \frac{a^{2}}{r^{2} a^{2}+2^{4 k}} \leq 4\left|1-r^{2}\right|,
$$

for any $a \in \mathbb{R}_{+}, k \in \mathbb{Z}_{+}$and $r \in[1 / 2,2]$, to bound the $L^{2}$ norm corresponding to a fixed $j \in \mathbb{Z}_{+}$in (6.17) by

$$
\left(1+4\left|1-r^{2}\right|\right)^{1 / 2}\left\|\frac{\eta_{j}(r(\tau-w(\xi)))}{\left|\tau-w(\xi)+i 2^{2 k}\right|} \eta_{k}\left(r^{\frac{1}{5}} \xi\right) \mathcal{F}\left[\eta_{0}^{r}\left(2^{2 k}(\cdot-\widetilde{t})\right) \widetilde{f}_{r}\right]\right\|_{L_{\xi, \tau}^{2}} .
$$

Now, the mean value theorem gives that

$$
\begin{aligned}
\left|\eta_{j}(r(\tau-w(\xi)))-\eta_{j}(\tau-w(\xi))\right| & \lesssim|r-1| \sup _{s \in[1, r]} 2^{j}\left|\eta_{j}^{\prime}(s(\tau-w(\xi)))\right| \\
& \lesssim|r-1| \sum_{\left|j^{\prime}-j\right| \leq 1, j^{\prime} \in \mathbb{Z}_{+}} \eta_{j^{\prime}}(\tau-w(\xi))
\end{aligned}
$$


if $r \in[3 / 4,5 / 4]$. Therefore, we deduce after gathering (6.16) over $j \in \mathbb{Z}_{+}$that

$$
\begin{aligned}
& \left\|P_{k} D_{1 / r}\left(\widetilde{f}_{r}\right)\right\|_{N_{k}} \\
& \quad \leq \varphi(r) \sup _{\widetilde{t} \in \mathbb{R}}\left\|\left(\tau-w(\xi)+i 2^{2 k}\right)^{-1} \eta_{k}\left(r^{\frac{1}{5}} \xi\right) \mathcal{F}\left[\eta_{0}^{r}\left(2^{2 k}(\cdot-\widetilde{t})\right) \widetilde{f}_{r}\right]\right\|_{X_{k}},
\end{aligned}
$$

where $\varphi$ is a continuous function defined in a neighborhood of 1 and satisfying $\lim _{r \rightarrow 1} \varphi(r)=1$. In order to deal with $\eta_{0}^{r}$ appearing on the right-hand side of (6.20), we get from the fundamental theorem of calculus that

$$
\eta_{0}^{r}\left(2^{2 k}(t-\widetilde{t})\right)-\eta_{0}\left(2^{2 k}(t-\widetilde{t})\right)=\int_{1}^{r^{-1}} \gamma_{s}\left(2^{2 k}(t-\widetilde{t})\right) d s,
$$

where $\gamma_{s}(t)=t \eta_{0}^{\prime}(s t)$. Moreover, we use that

$$
\eta_{0}\left(2^{2 k}\left(t-\left(\widetilde{t}+2 \cdot 2^{-2 k}\right)\right)\right)+\eta_{0}\left(2^{2 k}\left(t-\left(\widetilde{t}-2 \cdot 2^{-2 k}\right)\right)\right)=1
$$

on the support of the integral on the right-hand side of (6.21). Hence, it follows from Minkowski's inequality and estimates (2.23) and (6.20) that

$$
\begin{aligned}
& \left\|P_{k} D_{1 / r}\left(\tilde{f}_{r}\right)\right\|_{N_{k}} \\
& \quad \leq \widetilde{\varphi}(r) \sup _{\widetilde{t} \in \mathbb{R}}\left\|\left(\tau-w(\xi)+i 2^{2 k}\right)^{-1} \eta_{k}\left(r^{\frac{1}{5}} \xi\right) \mathcal{F}\left[\eta_{0}\left(2^{2 k}(\cdot-\widetilde{t})\right) \widetilde{f}_{r}\right]\right\|_{X_{k}},
\end{aligned}
$$

where $\widetilde{\varphi}$ is a function with similar properties as $\varphi$. Moreover, we observe by arguing as in (6.19) that

$$
\left.\mid \eta_{k}\left(r^{\frac{1}{5}} \xi\right)\right)-\eta_{k}(\xi)|\lesssim| r^{\frac{1}{5}}-1 \mid \sum_{\left|k^{\prime}-k\right| \leq 1, k^{\prime} \in \mathbb{Z}_{+}} \eta_{k^{\prime}}(\xi) .
$$

Thus, we deduce by gathering estimates (6.22) and (6.23) that

$$
\begin{aligned}
& \left\|P_{k} D_{1 / r}\left(\tilde{f}_{r}\right)\right\|_{N_{k}} \\
& \leq \widetilde{\varphi}(r)\left(1+\left|r^{\frac{1}{5}}-1\right|\right)\left\|P_{k} \widetilde{f}_{r}\right\|_{N_{k}} \\
& +\widetilde{\varphi}(r)\left|r^{\frac{1}{5}}-1\right| \sup _{\widetilde{t} \in \mathbb{R}}\left\|\left(\tau-w(\xi)+i 2^{2 k}\right)^{-1} \eta_{k-1}(\xi) \mathcal{F}\left[\eta_{0}\left(2^{2 k}(\cdot-\widetilde{t})\right) \widetilde{f}_{r}\right]\right\|_{X_{k-1}} \\
& +\widetilde{\varphi}(r)\left|r^{\frac{1}{5}}-1\right| \sup _{\widetilde{t} \in \mathbb{R}}\left\|\left(\tau-w(\xi)+i 2^{2 k}\right)^{-1} \eta_{k+1}(\xi) \mathcal{F}\left[\eta_{0}\left(2^{2 k}(\cdot-\widetilde{t})\right) \widetilde{f}_{r}\right]\right\|_{X_{k+1}} .
\end{aligned}
$$

To deal with the second term on the right-hand side of 6.24), we notice that $\left|\tau-w(\xi)+i 2^{2 k}\right|^{-1} \leq\left|\tau-w(\xi)+i 2^{2(k-1)}\right|^{-1}$ and $\eta_{0}\left(2^{2(k-1)}(\cdot-\widetilde{t})\right)=1$ on the support of $\eta_{0}\left(2^{2 k}(\cdot-\widetilde{t})\right)$. Then, it follows from estimate (2.23) that

(6.25) $\sup _{\widetilde{t} \in \mathbb{R}}\left\|\left(\tau-w(\xi)+i 2^{2 k}\right)^{-1} \eta_{k-1}(\xi) \mathcal{F}\left[\eta_{0}\left(2^{2 k}(\cdot-\widetilde{t})\right) \tilde{f}_{r}\right]\right\|_{X_{k-1}} \lesssim\left\|P_{k-1} \widetilde{f}_{r}\right\|_{N_{k-1}}$.

On the other hand, we have that $\left|\tau-w(\xi)+i 2^{2 k}\right|^{-1} \leq 4\left|\tau-w(\xi)+i 2^{2(k+1)}\right|^{-1}$. Moreover, we observe that

$$
2^{2(k+1)} \int_{-\tilde{t}-2^{4} \cdot 2^{-2(k+1)}}^{-\tilde{t}+2^{4} \cdot 2^{-2(k+1)}} \eta_{0}\left(2^{2(k+1)}(t+s)\right) d s=\int \eta_{0}(s) d s>0
$$


if $t$ lies in the support of $\eta_{0}\left(2^{2 k}(\cdot-\tilde{t})\right)$. Therefore, we deduce from Minkowski's inequality and by using estimate (2.23) that

$$
\sup _{\widetilde{t} \in \mathbb{R}}\left\|\left(\tau-w(\xi)+i 2^{2 k}\right)^{-1} \eta_{k+1}(\xi) \mathcal{F}\left[\eta_{0}\left(2^{2 k}(\cdot-\widetilde{t})\right) \tilde{f}_{r}\right]\right\|_{X_{k+1}} \lesssim\left\|P_{k+1} \widetilde{f}_{r}\right\|_{N_{k+1}} .
$$

We conclude the proof of estimate (6.15) by gathering estimates (6.24), (6.25), (6.26), summing over $k \in \mathbb{Z}_{+}$and recalling the definition of $N^{s}$ in (2.8).

The proof of estimate (6.12) follows in a similar way (it is actually easier).

Proof of Proposition 6.2. Fix $s \geq \frac{5}{4}$. First, it is worth noticing that we can always assume that the initial data $u_{0}$ have small $H^{s}$-norm by using a scaling argument.

Indeed, if $u$ is a solution to the IVP (1.1) on the time interval $[0, T]$, then $u_{\lambda}(x, t)=\lambda^{2} u\left(\lambda x, \lambda^{5} t\right)$ is also a solution to the equation (1.1) with initial data $u_{\lambda}(\cdot, 0)=\lambda^{2} u_{0}(\lambda \cdot)$ on the time interval $\left[0, \lambda^{-5} T\right]$. For $\epsilon>0$, let us denote by $\mathcal{B}^{s}(\epsilon)$ the ball of $H^{s}(\mathbb{R})$ centered at the origin with radius $\epsilon$. Since

$$
\left\|u_{\lambda}(\cdot, 0)\right\|_{H^{s}} \lesssim \lambda^{\frac{3}{2}}\left(1+\lambda^{s}\right)\left\|u_{0}\right\|_{H^{s}},
$$

we can always force $u_{\lambda}(\cdot, 0)$ to belong to $\mathcal{B}^{s}(\epsilon)$ by choosing $\lambda \sim \epsilon^{\frac{2}{3}}\left\|u_{0}\right\|_{H^{s}}^{-\frac{2}{3}}$. Therefore, it is enough to prove that if $u_{0} \in \mathcal{B}^{s}(\epsilon)$, then Proposition 6.2 holds with $T=1$. This would imply that Proposition 6.2 holds for arbitrarily large initial data in $H^{s}(\mathbb{R})$ with a time $T \sim \lambda^{5} \sim\left\|u_{0}\right\|_{H^{s}}^{-\frac{10}{3}}$.

Now, fix $u_{0} \in H^{\infty}(\mathbb{R}) \cap \mathcal{B}^{s}(\epsilon)$ and let $u \in C\left([-T, T] ; H^{\infty}\right)$ by the solution to (1.1) given by Theorem 6.1 where $0<T \leq 1$. We obtain by gathering the linear estimate (2.32), the bilinear estimates (4.1)-(4.2) and the energy estimate (5.6) that

$$
\Lambda_{T}^{\sigma}(u)^{2} \lesssim\left\|u_{0}\right\|_{H^{\sigma}}^{2}+\left(\Lambda_{T}^{s}(u)+\Lambda_{T}^{s}(u)^{2}\right) \Lambda_{T}^{\sigma}(u)^{2}
$$

for any $\sigma \geq s$ as soon as $\Lambda_{T}^{s}(u)<\widetilde{\delta}_{0}$. Here, $\widetilde{\delta}_{0}$ is a small positive number chosen 8 such that $\|u\|_{L_{T}^{\infty} H_{x}^{s}}<\delta_{0}$ as soon as $\Lambda_{T}^{s}(u)<\widetilde{\delta}_{0}$, where $\delta_{0}$ is given by Corollary 5.4. Estimates (2.32), 6.27) with $\sigma=s$, Lemma 6.3 and a continuity argument ensure the existence of $\epsilon_{s}>0$ and $C_{s}>0$ such that $\Gamma_{T}^{s}(u) \leq C_{s} \epsilon$ provided $\left\|u_{0}\right\|_{H^{s}} \leq \epsilon \leq \epsilon_{s}$ where $\Gamma_{T}^{s}(u)$ is defined by

$$
\Gamma_{T}^{s}(u):=\max \left\{\|u\|_{B^{s}(T)},\|u\|_{F^{s}(T)}\right\} .
$$

Thus, estimates (2.10), (2.32) and (6.27) yield

$$
\|u\|_{L_{T}^{\infty} H_{x}^{\sigma}} \lesssim \Gamma_{T}^{\sigma}(u) \lesssim\left\|u_{0}\right\|_{H^{\sigma}}
$$

for all $\sigma \geq s$, provided $\left\|u_{0}\right\|_{H^{s}} \leq \epsilon \leq \epsilon_{s}$.

Therefore, using estimate (6.29) with $\sigma=4$ we can reapply the result of Theorem 6.1 a finite number of times and extend the solution $u$ on the time interval $[-1,1]$. This concludes the proof of Proposition 6.2

\section{2. $L^{2}$ - Lipschitz bound for the difference of two solutions and unique-}

ness. Let $u_{1}$ and $u_{2}$ be two solutions of the equation (1.1) defined on a time interval $[-T, T]$ for some $0<T \leq 1$ and with respective initial data $u_{1}(\cdot, 0)=\varphi_{1}$ and $u_{2}(\cdot, 0)=\varphi_{2}$. We also assume that $\varphi_{1}, \varphi_{2} \in \mathcal{B}^{2}(\epsilon)$ and

$$
\Gamma_{T}^{2}\left(u_{i}\right) \leq C_{2} \epsilon \leq C_{2} \epsilon_{2}, \quad \text { for } i=1,2,
$$

\footnotetext{
${ }^{8}$ The choice is possible by using estimates (2.10) and (2.32).
} 
where $\Gamma_{T}^{2}(\cdot)$ is defined in (6.28). Moreover, according to (2.10), we can choose $\epsilon$ small enough such that $\left\|u_{i}\right\|_{L_{T}^{\infty} H_{x}^{2}}<\delta_{1}$ where $\delta_{1}$ is given in Corollary 5.11.

Let us define $v$ by $v=u_{1}-u_{2}$. Observe that $v$ is a solution to equation (5.44) and also to

$$
\partial_{t} v=\partial_{x}^{5} v+c_{1} \partial_{x}\left(\partial_{x}\left(u_{1}+u_{2}\right) \partial_{x} v\right)+c_{2} \partial_{x}\left(u_{1} \partial_{x}^{2} v\right)+c_{2} \partial_{x}\left(v \partial_{x}^{2} u_{2}\right) .
$$

Then, we conclude by gathering estimates (2.32), (4.3), (4.4) and (5.50) that there exists $0<\widetilde{\epsilon}_{2} \leq \epsilon_{2}$ such that

$$
\Gamma_{T}^{0}(v) \lesssim\left\|\varphi_{1}-\varphi_{2}\right\|_{L^{2}}
$$

provided $u_{1}$ and $u_{2}$ satisfy (6.30) with $0<\epsilon \leq \widetilde{\epsilon}_{2}$

We now state our uniqueness result.

Proposition 6.4. Let $u_{1}$ and $u_{2}$ be two solutions to the equation (1.1) in the class (1.9) with $s=2$, defined on a time interval $[-T, T]$ for some $T>0$ and satisfying $u_{1}(\cdot, 0)=u_{2}(\cdot, 0)=\varphi$. Then $u_{1}=u_{2}$ on $[-T, T]$.

Proof. Let us define $K:=\max \left\{\Gamma_{T}^{2}\left(u_{1}\right), \Gamma_{T}^{2}\left(u_{2}\right)\right\}$. As in the proof of Proposition 6.2. we use the scaling property of (1.1) and define $u_{i, \lambda}=\lambda^{2} u_{i}\left(\lambda x, \lambda^{5} t\right)$, for $i=1,2$ and $\lambda>0$, which are also solutions to the equation (1.1) on the time interval $[-S, S]$ with $S=\lambda^{-5} T$ and with initial data $\varphi_{\lambda}=\lambda^{2} \varphi(\lambda \cdot)$. Moreover, since

$$
\left\|u_{i, \lambda}\right\|_{L_{S}^{\infty} H_{x}^{2}}+\left\|u_{i, \lambda}\right\|_{B^{2}(S)} \lesssim \lambda^{\frac{3}{2}}\left(1+\lambda^{2}\right)\left(\left\|u_{i}\right\|_{L_{T}^{\infty} H_{x}^{2}}+\left\|u_{i}\right\|_{B^{2}(T)}\right) \lesssim \lambda^{\frac{3}{2}}\left(1+\lambda^{2}\right) K,
$$

for $i=1,2$, we can always choose $\lambda=\lambda(K)$ small enough such that

$$
\left\|\varphi_{\lambda}\right\|_{H^{2}} \leq \epsilon,\left\|u_{i, \lambda}\right\|_{B^{2}(S)} \leq C_{2} \epsilon /(3 c) \leq C_{2} \tilde{\epsilon}_{2} /(3 c) \text { and }\left\|u_{i, \lambda}\right\|_{F^{2}(S)} \leq C(K),
$$

for $i=1,2$, and where $c$ is the implicit constant appearing in the first inequality of (6.34) below.

Since $\left\|u_{i, \lambda}\right\|_{F^{2}(S)}<\infty$, there exists $n \in \mathbb{Z}_{+}$such that

$$
\left\|P_{>n} u_{i, \lambda}\right\|_{F^{2}(S)} \leq C_{2} \epsilon / 3, \quad i=1,2 .
$$

On the other hand, we deduce from (2.32), (6.8) and (6.32) that

$$
\begin{aligned}
\left\|P_{\leq n} u_{i, \lambda}\right\|_{F^{2}(S)} \lesssim & \left\|u_{i, \lambda}\right\|_{B^{2}(S)}+S^{\frac{1}{2}} 2^{2 n}\left\|P_{\leq n} \partial_{x}\left(\left(\partial_{x} u_{i, \lambda}\right)^{2}\right)\right\|_{L_{S}^{\infty} L_{x}^{2}} \\
& +S^{\frac{1}{2}} 2^{2 n}\left\|P_{\leq n} \partial_{x}\left(u_{i, \lambda} \partial_{x}^{2} u_{i, \lambda}\right)\right\|_{L_{S}^{\infty} L_{x}^{2}} \\
\lesssim & C_{2} \epsilon / 3+S^{\frac{1}{2}} 2^{3 n}\left\|u_{i, \lambda}\right\|_{L_{S}^{\infty} H_{x}^{2}}^{2}
\end{aligned}
$$

By choosing $S_{1}=S_{1}(K)$ small enough, we deduce from (6.32) -6.34) that $u_{\lambda, 1}$ and $u_{\lambda, 2}$ satisfy the smallness condition (6.30) on $\left[-S_{1}, S_{1}\right]$, i.e.

$$
\Gamma_{S_{1}}^{2}\left(u_{i, \lambda}\right) \leq C_{2} \epsilon \leq C_{2} \widetilde{\epsilon}_{2}, \quad \text { for } i=1,2 .
$$

This implies from (6.31) that $u_{1, \lambda} \equiv u_{2, \lambda}$ on $\left[-S_{1}, S_{1}\right]$. By applying this argument a finite number of times, we see that the equality holds in fact in $[-S, S]$. Then it follows after changing variables that $u_{1} \equiv u_{2}$ on $[-T, T]$. 
6.3. Existence. Let $2 \leq s<4$ and $u_{0} \in H^{s}(\mathbb{R})$. By using a scaling argument as in the proof of Proposition 6.2 we can assume that $u_{0} \in \mathcal{B}^{s}(\epsilon)$, with $\epsilon<\widetilde{\epsilon}_{s} \leq$ $\min \left(\epsilon_{s}, \frac{C_{2}}{C_{s}} \widetilde{\epsilon}_{2}\right)$. Note here that $\widetilde{\epsilon}_{s}$ will be determined later.

We will use the Bona-Smith argument (cf. 3]). Let $\rho \in \mathcal{S}(\mathbb{R})$ with $\rho \geq 0$, $\int \rho d x=1$, and $\int x^{k} \rho(x) d x=0, k \in \mathbb{Z}_{+}, 0 \leq k \leq[s]+1$. For any $\lambda>0$, define $\rho_{\lambda}(x)=\lambda^{-1} \rho\left(\lambda^{-1} x\right)$. The following lemma, whose proof can be found in [3] (see also Proposition 2.1 in [17]), gathers the properties of the smoothing operators which will be used in this section.

Lemma 6.5. Let $s \geq 0, \phi \in H^{s}(\mathbb{R})$ and for any $\lambda>0, \phi_{\lambda}=\rho_{\lambda} * \phi$. Then,

$$
\left\|\phi_{\lambda}\right\|_{H^{s+\alpha}} \lesssim \lambda^{-\alpha}\|\phi\|_{H^{s}}, \quad \forall \alpha \geq 0
$$

and

$$
\left\|\phi-\phi_{\lambda}\right\|_{H^{s-\beta}} \underset{\lambda \rightarrow 0}{=} o\left(\lambda^{\beta}\right), \quad \forall \beta \in[0, s] .
$$

Now we regularize the initial data by letting $u_{0, \lambda}=\rho_{\lambda} * u_{0}$. Since $u_{0, \lambda} \in H^{\infty}(\mathbb{R})$, we deduce from Theorem 6.1 that for any $\lambda>0$, there exist a positive time $T_{\lambda}$ and a unique solution

$$
u_{\lambda} \in C\left(\left[-T_{\lambda}, T_{\lambda}\right] ; H^{\infty}(\mathbb{R})\right) \quad \text { satisfying } \quad u_{\lambda}(\cdot, 0)=u_{0, \lambda} .
$$

We observe that $\left\|u_{0, \lambda}\right\|_{H^{s}} \leq\left\|u_{0}\right\|_{H^{s}} \leq \epsilon$. Thus, it follows from the proof of Proposition 6.2 and estimate (6.35) that the sequence of solutions $\left\{u_{\lambda}\right\}$ can be extended on the time interval $[-1,1]$ and satisfies

$$
\begin{gathered}
\Gamma_{1}^{s}\left(u_{\lambda}\right) \leq C_{s} \epsilon \leq \min \left(C_{2} \widetilde{\epsilon}_{2}, C_{s} \widetilde{\epsilon}_{s}\right) \\
\Gamma_{1}^{s}\left(u_{\lambda}\right) \lesssim\left\|u_{0}\right\|_{H^{s}} \quad \text { and } \quad \Gamma_{1}^{s+2}\left(u_{\lambda}\right) \lesssim\left\|u_{0, \lambda}\right\|_{H^{s+2}} \lesssim \lambda^{-2}\left\|u_{0}\right\|_{H^{s}}
\end{gathered}
$$

for all $\lambda>0$.

Then, we deduce from (6.31) and (6.36) that for any $0<\lambda^{\prime}<\lambda$,

$$
\Gamma_{1}^{0}\left(u_{\lambda}-u_{\lambda^{\prime}}\right) \lesssim\left\|u_{0, \lambda}-u_{0, \lambda^{\prime}}\right\|_{L_{x}^{2}} \underset{\lambda \rightarrow 0}{=} o\left(\lambda^{s}\right) .
$$

Moreover, we obtain by gathering estimates (2.32), (4.1)-(4.2), (5.51), (6.37) and choosing $\widetilde{\epsilon}_{s}$ small enough that

$$
\Gamma_{1}^{s}\left(u_{\lambda}-u_{\lambda^{\prime}}\right) \lesssim\left\|u_{0, \lambda}-u_{0, \lambda^{\prime}}\right\|_{H_{x}^{s}}+\Gamma_{1}^{s+2}\left(u_{\lambda}\right) \Gamma_{1}^{0}\left(u_{\lambda}-u_{\lambda^{\prime}}\right),
$$

since $s \geq 2$. This combined with (6.36), (6.38) and (6.39) yields

$$
\left\|u_{\lambda}-u_{\lambda^{\prime}}\right\|_{L_{1}^{\infty} H_{x}^{s}} \lesssim \Gamma_{1}^{s}\left(u_{\lambda}-u_{\lambda^{\prime}}\right) \underset{\lambda \rightarrow 0}{\longrightarrow} 0 .
$$

Therefore, we conclude that $\left\{u_{\lambda}\right\}$ converges in the norm $\Gamma_{1}^{s}$ to a solution $u$ of (1.1) in the class (1.9).

Remark 6.6. Observe that the convergence of $\left\{u_{\lambda}\right\}$ in $C\left([-1,1] ; H^{1}(\mathbb{R})\right)$ would be enough to obtain that the limit $u$ satisfies the equation (1.1) in the weak sense. 
6.4. Continuity of the flow map data-solution. Observe that for $s \geq 4$, the result was already proved in Theorem 3.1 in 33 . Then it is enough to prove it for $2 \leq s<4$. Let $u_{0} \in H^{s}(\mathbb{R})$. Once again we can assume by using a scaling argument that $u_{0} \in \mathcal{B}^{s}(\epsilon)$ with $0<\epsilon \leq \bar{\epsilon}<\widetilde{\epsilon}_{s}$ and where $\widetilde{\epsilon}_{s}$ was determined in the previous subsection. Then, the solution $u$ emanating from $u_{0}$ is defined on the time interval $[-1,1]$ and satisfies $u \in C\left([-1,1] ; H^{s}(\mathbb{R})\right)$.

Let $\theta>0$ be given. It suffices to prove that for any initial data $v_{0} \in \mathcal{B}^{s}(\epsilon)$ with $\left\|u_{0}-v_{0}\right\|_{H^{s}} \leq \delta$, where $\delta=\delta(\theta)>0$ will be fixed later, the solution $v \in$ $C\left([-1,1] ; H^{s}(\mathbb{R})\right)$ emanating from $v_{0}$ satisfies

$$
\|u-v\|_{L_{1}^{\infty} H_{x}^{s}} \leq \theta .
$$

For any $\lambda>0$, we normalize the initial data $u_{0}$ and $v_{0}$ by defining $u_{0, \lambda}=\rho_{\lambda} * u_{0}$ and $v_{0, \lambda}=\rho_{\lambda} * v_{0}$ as in the previous subsection and consider the associated smooth solutions $u_{\lambda}, v_{\lambda} \in C\left([-1,1] ; H^{\infty}(\mathbb{R})\right)$. Then it follows from the triangle inequality that

$$
\|u-v\|_{L_{1}^{\infty} H_{x}^{s}} \leq\left\|u-u_{\lambda}\right\|_{L_{1}^{\infty} H_{x}^{s}}+\left\|u_{\lambda}-v_{\lambda}\right\|_{L_{1}^{\infty} H_{x}^{s}}+\left\|v-v_{\lambda}\right\|_{L_{1}^{\infty} H_{x}^{s}} .
$$

On the one hand, according to (6.41), we can choose $\lambda_{0}$ small enough so that

$$
\left\|u-u_{\lambda_{0}}\right\|_{L_{1}^{\infty} H_{x}^{s}}+\left\|v-v_{\lambda_{0}}\right\|_{L_{1}^{\infty} H_{x}^{s}} \leq 2 \theta / 3 \text {. }
$$

On the other hand, we get from (6.35) that

$$
\left\|u_{0, \lambda_{0}}-v_{0, \lambda_{0}}\right\|_{H^{4}} \lesssim \lambda_{0}^{-(4-s)}\left\|u_{0}-v_{0}\right\|_{H^{4}} \lesssim \lambda_{0}^{-(4-s)} \delta .
$$

Therefore, by using the continuity of the flow map for smooth initial data (cf. Theorem 3.1 in [33]), we can choose $\delta>0$ small enough such that

$$
\left\|u_{\lambda_{0}}-v_{\lambda_{0}}\right\|_{L_{1}^{\infty} H_{x}^{s}} \leq \theta / 3 .
$$

Estimate (6.42) is concluded by gathering (6.43)-(6.45).

\section{Appendix: How to DeAl With the Cubic term $\partial_{x}\left(u^{3}\right)$}

In this appendix, we explain what are the main modifications needed to deal with the cubic term $\partial_{x}\left(u^{3}\right)$ (i.e. in the case where $c_{3} \neq 0$ ). As above, we fix $\alpha=2$ in the definition of the spaces $F_{\alpha}^{s}(T), N_{\alpha}^{s}(T), F_{\alpha}^{s}, N_{\alpha}^{s}, F_{k, \alpha}, N_{k, \alpha}$ and write those spaces without the index $\alpha=2$, since there is no risk of confusion.

7.1. Short time trilinear estimate. In this subsection, we prove the trilinear estimate for the nonlinear term $\partial_{x}\left(u^{3}\right)$.

Proposition 7.1. Let $s \geq 0$ and $T \in(0,1]$ be given. Then, it holds that

$$
\begin{aligned}
\left\|\partial_{x}(u v w)\right\|_{N^{s}(T)} \lesssim & \|u\|_{F^{0}(T)}\|v\|_{F^{0}(T)}\|w\|_{F^{s}(T)}+\|u\|_{F^{0}(T)}\|w\|_{F^{0}(T)}\|v\|_{F^{s}(T)} \\
& +\|v\|_{F^{0}(T)}\|w\|_{F^{0}(T)}\|u\|_{F^{s}(T)}
\end{aligned}
$$

for all $u, v, w \in F^{s}(T)$.

We split the proof of Proposition 7.1 into several technical lemmas depending on the frequency interactions.

Lemma 7.2 (high $\times$ low $\times$ low $\rightarrow$ high). Assume that $k, k_{1}, k_{2}, k_{3} \in \mathbb{Z}_{+}$satisfy $k \geq 20,\left|k_{3}-k\right| \leq 5$ and $0 \leq k_{1} \leq k_{2} \leq k_{3}-10$. Then,

$$
\left\|P_{k} \partial_{x}\left(u_{k_{1}} v_{k_{2}} w_{k_{3}}\right)\right\|_{N_{k}} \lesssim 2^{-3 k / 2}\left\|u_{k_{1}}\right\|_{F_{k_{1}}}\left\|v_{k_{2}}\right\|_{F_{k_{2}}}\left\|w_{k_{3}}\right\|_{F_{k_{3}}}
$$

for all $u_{k_{1}} \in F_{k_{1}}, v_{k_{2}} \in F_{k_{2}}$ and $w_{k_{3}} \in F_{k_{3}}$. 
Proof. Arguing exactly as in the proof of Lemma 4.3, it suffices to prove that

$$
\begin{aligned}
2^{k} \sum_{j \geq 2 k} 2^{-j / 2} \| \mathbf{1}_{D_{k, j}} & \cdot\left(f_{k_{1}, j_{1}} * f_{k_{2}, j_{2}} * f_{k_{3}, j_{3}}\right) \|_{L_{\xi, \tau}^{2}} \\
& \lesssim 2^{j_{1} / 2}\left\|f_{k_{1}, j_{1}}\right\|_{L^{2}} 2^{j_{2} / 2}\left\|f_{k_{2}, j_{2}}\right\|_{L^{2}} 2^{j_{3} / 2}\left\|f_{k_{3}, j_{3}}\right\|_{L^{2}}
\end{aligned}
$$

where the functions $f_{k_{i}, j_{i}}$ are localized in $D_{k_{i}, j_{i}}$, with $j_{i} \geq 2 k$, for $i=1,2,3$.

But, we deduce from estimates (3.29) and (3.31) that

$$
\begin{aligned}
& 2^{k} \sum_{j \geq 2 k} 2^{-j / 2}\left\|\mathbf{1}_{D_{k, j}} \cdot\left(f_{k_{1}, j_{1}} * f_{k_{2}, j_{2}} * f_{k_{3}, j_{3}}\right)\right\|_{L_{\xi, \tau}^{2}} \\
& \quad \lesssim 2^{k} \sum_{j \geq 2 k} 2^{-j / 2} 2^{k_{1} / 2} 2^{-2 k} 2^{j_{1} / 2}\left\|f_{k_{1}, j_{1}}\right\|_{L^{2}} 2^{j_{2} / 2}\left\|f_{k_{2}, j_{2}}\right\|_{L^{2}} 2^{j_{3} / 2}\left\|f_{k_{3}, j_{3}}\right\|_{L^{2}}
\end{aligned}
$$

which implies estimate (7.3) after summing over $j$.

Lemma 7.3 (high $\times$ high $\times$ low $\rightarrow$ high). Assume that $k, k_{1}, k_{2}, k_{3} \in \mathbb{Z}_{+}$satisfy $k \geq 20,\left|k_{3}-k\right| \leq 5, k_{3}-10 \leq k_{2} \leq k_{3}$ and $0 \leq k_{1} \leq k_{2}-20$. Then,

$$
\left\|P_{k} \partial_{x}\left(u_{k_{1}} v_{k_{2}} w_{k_{3}}\right)\right\|_{N_{k}} \lesssim 2^{-k}\left\|u_{k_{1}}\right\|_{F_{k_{1}}}\left\|v_{k_{2}}\right\|_{F_{k_{2}}}\left\|w_{k_{3}}\right\|_{F_{k_{3}}},
$$

for all $u_{k_{1}} \in F_{k_{1}}, v_{k_{2}} \in F_{k_{2}}$ and $w_{k_{3}} \in F_{k_{3}}$.

Proof. Once again, it is enough to prove that estimate (7.3) remains true in this case. According to the frequency localization, we have that $\widetilde{\Omega} \sim 2^{5 k_{\max }}$, where $\widetilde{\Omega}$ is defined in (3.32). This yields $j_{\max } \geq 5 k-20$. Therefore, it follows from estimate (3.45) that

$$
\begin{aligned}
& 2^{k} \sum_{j \geq 2 k} 2^{-j / 2}\left\|\mathbf{1}_{D_{k, j}} \cdot\left(f_{k_{1}, j_{1}} * f_{k_{2}, j_{2}} * f_{k_{3}, j_{3}}\right)\right\|_{L_{\xi, \tau}^{2}} \\
& \quad \lesssim 2^{k} \sum_{j \geq 2 k} 2^{-j / 2} 2^{\left(j_{1}+j_{2}+j_{3}+j\right) / 2} 2^{\left(k_{1}+k_{2}\right) / 2} 2^{-\left(j_{\max }+j_{s u b}\right) / 2} \prod_{i=1}^{3}\left\|f_{k_{i}, j_{i}}\right\|_{L^{2}},
\end{aligned}
$$

which provides the bound in estimate (7.3) in both cases $j_{\max }=j$ and $j_{\max } \neq j_{\max }$. This finishes the proof of Lemma 7.3 .

Lemma 7.4 (high $\times$ high $\times$ high $\rightarrow$ high). Assume that $k, k_{1}, k_{2}, k_{3} \in \mathbb{Z}_{+}$satisfy $k \geq 20,\left|k_{3}-k\right| \leq 5, k_{3}-10 \leq k_{2} \leq k_{3}$ and $k_{2}-30 \leq k_{1} \leq k_{2}$. Then,

$$
\left\|P_{k} \partial_{x}\left(u_{k_{1}} v_{k_{2}} w_{k_{3}}\right)\right\|_{N_{k}} \lesssim\left\|u_{k_{1}}\right\|_{F_{k_{1}}}\left\|v_{k_{2}}\right\|_{F_{k_{2}}}\left\|w_{k_{3}}\right\|_{F_{k_{3}}},
$$

for all $u_{k_{1}} \in F_{k_{1}}, v_{k_{2}} \in F_{k_{2}}$ and $w_{k_{3}} \in F_{k_{3}}$.

Proof. We argue exactly as in the proof of Lemma 7.3 and observe that estimate (7.5) leads to estimate (7.3) even without using that $j_{\max } \geq 5 k-20$, which is not always satisfied in this case. Instead, it is sufficient to use that $j, j_{i} \geq 2 k$ for all $i=1,2,3$.

Lemma 7.5 (high $\times$ high $\times$ high $\rightarrow$ low). Assume that $k, k_{1}, k_{2}, k_{3} \in \mathbb{Z}_{+}$satisfy $k_{3}-5 \leq k_{2} \leq k_{3}, k_{2}-10 \leq k_{1} \leq k_{2}$ and $20 \leq k \leq k_{1}-10$. Then,

$$
\left\|P_{k} \partial_{x}\left(u_{k_{1}} v_{k_{2}} w_{k_{3}}\right)\right\|_{N_{k}} \lesssim 2^{-\left(k_{3}+k\right) / 2}\left\|u_{k_{1}}\right\|_{F_{k_{1}}}\left\|v_{k_{2}}\right\|_{F_{k_{2}}}\left\|w_{k_{3}}\right\|_{F_{k_{3}}},
$$

for all $u_{k_{1}} \in F_{k_{1}}, v_{k_{2}} \in F_{k_{2}}$ and $w_{k_{3}} \in F_{k_{3}}$. 
Proof. We argue as in the proof of Lemma 4.7. Thus it is enough to prove that

$$
\begin{aligned}
2^{2\left(k_{3}-k\right)} 2^{k} \sum_{j \geq 0} 2^{-j / 2} \| \mathbf{1}_{D_{k, j}} & \cdot\left(f_{k_{1}, j_{1}}^{m} * f_{k_{2}, j_{2}}^{m} * f_{k_{3}, j_{3}}^{m}\right) \|_{L_{\xi, \tau}^{2}} \\
& \lesssim 2^{j_{1} / 2}\left\|f_{k_{1}, j_{1}}^{m}\right\|_{L^{2}} 2^{j_{2} / 2}\left\|f_{k_{2}, j_{2}}^{m}\right\|_{L^{2}} 2^{j_{3} / 2}\left\|f_{k_{3}, j_{3}}^{m}\right\|_{L^{2}},
\end{aligned}
$$

where the functions $f_{k_{i}, j_{i}}^{m}$ are localized in $D_{k_{i}, j_{i}}$, with $j_{i} \geq 2 k_{3}$, for $i=1,2,3$. According to estimate (3.45), we can bound the left-hand side of (17.8) by

$$
2^{2 k_{3}-k} \sum_{j \geq 0} 2^{-j / 2} 2^{\left(j_{1}+j_{2}+j_{3}+j\right) / 2} 2^{\left(k+k_{1}\right) / 2} 2^{-\left(j_{\max }+j_{s u b}\right) / 2} \prod_{i=1}^{3}\left\|f_{k_{i}, j_{i}}\right\|_{L^{2}} .
$$

Moreover, we have $\widetilde{\Omega} \sim 2^{5 k_{\max }}$ in this case, so that $j_{\max } \geq 5 k_{3}-20$. This implies estimate (7.8) in both cases $j=j_{\max }$ and $j \neq j_{\max }$.

Lemma 7.6 (high $\times$ high $\times$ low $\rightarrow$ low). Assume that $k, k_{1}, k_{2}, k_{3} \in \mathbb{Z}_{+}$satisfy $k \geq 20, k_{3}-5 \leq k_{2} \leq k_{3}$ and $0 \leq k_{1}, k \leq k_{2}-10$. Then,

$$
\left\|P_{k} \partial_{x}\left(u_{k_{1}} v_{k_{2}} w_{k_{3}}\right)\right\|_{N_{k}} \lesssim 2^{-\left(k_{3}+k\right) / 2}\left\|u_{k_{1}}\right\|_{F_{k_{1}}}\left\|v_{k_{2}}\right\|_{F_{k_{2}}}\left\|w_{k_{3}}\right\|_{F_{k_{3}}},
$$

for all $u_{k_{1}} \in F_{k_{1}}, v_{k_{2}} \in F_{k_{2}}$ and $w_{k_{3}} \in F_{k_{3}}$.

Proof. Following the proof of Lemma 7.5, we need to prove that estimate (7.8) still holds in this case. This is a direct consequence of estimates (3.47) and (3.48).

Lemma 7.7 (low $\times$ low $\times$ low $\rightarrow$ low). Assume that $k, k_{1}, k_{2}, k_{3} \in \mathbb{Z}_{+}$satisfy $0 \leq k, k_{1}, k_{2}, k_{3} \leq 200$. Then,

$$
\left\|P_{k} \partial_{x}\left(u_{k_{1}} v_{k_{2}} w_{k_{3}}\right)\right\|_{N_{k}} \lesssim\left\|u_{k_{1}}\right\|_{F_{k_{1}}}\left\|v_{k_{2}}\right\|_{F_{k_{2}}}\left\|w_{k_{3}}\right\|_{F_{k_{3}}},
$$

for all $u_{k_{1}} \in F_{k_{1}}, v_{k_{2}} \in F_{k_{2}}$ and $w_{k_{3}} \in F_{k_{3}}$.

Proof. It follows by arguing as in Lemma 4.10.

Finally, we give the proof of Proposition 7.1 .

Proof of Proposition 7.1. Fix $s \geq 0$. We choose two extensions $\tilde{u}, \tilde{v}$ and $\tilde{w}$ of $u, v$ and $w$ satisfying

$$
\|\tilde{u}\|_{F^{s}} \leq 2\|u\|_{F^{s}(T)}, \quad\|\tilde{v}\|_{F^{s}} \leq 2\|v\|_{F^{s}(T)} \quad \text { and } \quad\|\tilde{w}\|_{F^{s}} \leq 2\|w\|_{F^{s}(T)} .
$$

Therefore $\partial_{x}(\tilde{u} \tilde{v} \tilde{w})$ is an extension of $\partial_{x}(u v w)$ on $\mathbb{R}^{2}$, and we have from the definition of $N^{s}(T)$ and Minkowski inequality that

$$
\left\|\partial_{x}(u v w)\right\|_{N^{s}(T)} \leq\left(\sum_{k \geq 0} 2^{2 k s}\left(\sum_{k_{1}, k_{2}, k_{3} \geq 0}\left\|P_{k} \partial_{x}\left(P_{k_{1}} \tilde{u} P_{k_{2}} \tilde{v} P_{k_{3}} \tilde{w}\right)\right\|_{N_{k}}\right)^{2}\right)^{\frac{1}{2}} .
$$

Note that by symmetry, we can always assume that $0 \leq k_{1} \leq k_{2} \leq k_{3}$. Moreover, we denote

$$
\begin{aligned}
& G_{1}=\left\{\left(k_{1}, k_{2}, k_{3}\right) \in \mathbb{Z}_{+}^{3}: k \geq 20,\left|k_{3}-k\right| \leq 5,0 \leq k_{1} \leq k_{2} \leq k_{3}-10\right\}, \\
& G_{2}=\left\{\left(k_{1}, k_{2}, k_{3}\right) \in \mathbb{Z}_{+}^{3}: k \geq 20,\left|k_{3}-k\right| \leq 5\left|k_{3}-k_{2}\right| \leq 10,0 \leq k_{1} \leq k_{2}-20\right\} \text {, } \\
& G_{3}=\left\{\left(k_{1}, k_{2}, k_{3}\right) \in \mathbb{Z}_{+}^{3}: k \geq 20,\left|k_{3}-k\right| \leq 5\left|k_{3}-k_{2}\right| \leq 10,\left|k_{1}-k_{2}\right| \leq 30\right\}, \\
& G_{4}=\left\{\left(k_{1}, k_{2}, k_{3}\right) \in \mathbb{Z}_{+}^{3}: k_{3}-5 \leq k_{2} \leq k_{3}, k_{2}-10 \leq k_{1} \leq k_{2}, 20 \leq k \leq k_{1}-10\right\} \text {, } \\
& G_{5}=\left\{\left(k_{1}, k_{2}, k_{3}\right) \in \mathbb{Z}_{+}^{3}: k_{3}-5 \leq k_{2} \leq k_{3}, 20 \leq k, k_{1} \leq k_{2}-10\right\} \text {, } \\
& G_{6}=\left\{\left(k_{1}, k_{2}, k_{3}\right) \in \mathbb{Z}_{+}^{3}: 0 \leq k, k_{1}, k_{2}, k_{3} \leq 200\right\} \text {. }
\end{aligned}
$$


Note that for a given $k \in \mathbb{Z}_{+}$, some of these regions may be empty and others may overlap, but due to the frequency localization, we always have that

$$
\left\|\partial_{x}(u v w)\right\|_{N^{s}(T)} \leq \sum_{i=1}^{6}\left(\sum_{k \geq 0} 2^{2 k s}\left(\sum_{\left(k_{1}, k_{2}, k_{3}\right) \in G_{i}}\left\|P_{k} \partial_{x}\left(P_{k_{1}} \tilde{u} P_{k_{2}} \tilde{v} P_{k_{3}} \tilde{w}\right)\right\|_{N_{k}}\right)^{2}\right)^{\frac{1}{2}} \text {. }
$$

We conclude the proof of Proposition 7.1 by applying respectively Lemmas 7.27 .7 to each of the sums appearing on the right-hand side of (7.11).

7.2. Modifications to the energy estimates. We only explain how to deal with the $a$ priori estimates, since the modifications would be similar to derive estimates for the differences of two solutions. The main point is to derive an analog to Proposition 5.3 in the case where $c_{3} \neq 0$.

Proposition 7.8. Assume $s \geq \frac{5}{4}$ and $T \in(0,1]$. Then, if $u \in C\left([-T, T] ; H^{\infty}(\mathbb{R})\right)$ is a solution to (1.1), we have that

$$
E_{T}^{s}(u) \lesssim\left(1+\left\|u_{0}\right\|_{H^{s}}\right)\left\|u_{0}\right\|_{H^{s}}^{2}+\left(1+\Gamma_{T}^{s}(u)+\Gamma_{T}^{s}(u)^{2}\right) \Gamma_{T}^{s}(u)^{3},
$$

where

$$
\Gamma_{T}^{s}(u):=\max \left\{\|u\|_{F^{s}(T)},\|u\|_{B^{s}(T)}\right\} .
$$

Proof. The proof of Proposition 7.8 follows the same strategy as the one of Proposition 5.3. The unique difference is that we need to add the terms $\mathcal{M}_{k}^{1}(u), \alpha \mathcal{M}_{k}^{1}(u)$ and $\beta \mathcal{M}_{k}^{2}(u)$ to the right-hand side of (5.23), where

$$
\begin{gathered}
\mathcal{K}_{k}(u)=2 c_{3} \int_{\mathbb{R}} P_{k} u P_{k} \partial_{x}\left(u^{3}\right) d x \\
\mathcal{M}_{k}^{1}(u)=c_{3} \int_{\mathbb{R}} \partial_{x}\left(u^{3}\right) P_{k} \partial_{x}^{-1} u Q_{k} \partial_{x}^{-1} u d x+c_{3} \int_{\mathbb{R}} u P_{k}\left(u^{3}\right) Q_{k} \partial_{x}^{-1} u d x \\
+c_{3} \int_{\mathbb{R}} u P_{k} \partial_{x}^{-1} u Q_{k}\left(u^{3}\right) d x
\end{gathered}
$$

and

$$
\mathcal{M}_{k}^{2}(u)=c_{3} \int_{\mathbb{R}} \partial_{x}\left(u^{3}\right) P_{k} \partial_{x}^{-1} u P_{k} \partial_{x}^{-1} u d x+2 c_{3} \int_{\mathbb{R}} u P_{k}\left(u^{3}\right) P_{k} \partial_{x}^{-1} u d x .
$$

Therefore, it suffices to bound

$$
\sum_{k \geq 1} 2^{2 k s} \sup _{t_{k} \in[0, T]}\left|\int_{\left[0, t_{k}\right]}\left(\mathcal{K}_{k}(u)+\alpha \mathcal{M}_{k}^{1}(u)+\beta \mathcal{M}_{k}^{2}(u)\right) d t\right|
$$

by the the terms appearing on the right-hand side of (7.12).

We first treat the fourth-order term corresponding to $\mathcal{K}_{k}(u)$. We perform the same dyadic decomposition as in the proof of Proposition 7.1. Thus,

$$
\begin{aligned}
& \sum_{k \geq 1} 2^{2 k s} \sup _{t_{k} \in[0, T]}\left|\int_{\left[0, t_{k}\right]} \mathcal{K}_{k}(u) d t\right| \\
& \lesssim \sum_{i=1}^{6} \sum_{k \geq 1} 2^{2 k s} \sum_{\left(k_{1}, k_{2}, k_{3}\right) \in G_{i}} \sup _{t_{k} \in[0, T]}\left|\int_{\mathbb{R} \times\left[0, t_{k}\right]} P_{k} u P_{k} \partial_{x}\left(P_{k_{1}} u P_{k_{2}} u P_{k_{3}} u\right) d x\right| .
\end{aligned}
$$


By using respectively estimate (5.20) for the sums over $G_{1}$ and $G_{5}$ and estimate (5.21) for the sums over $G_{2}$ and $G_{4}$, the corresponding terms on the right-hand side of (17.14) can be bounded by

$$
\|u\|_{F^{0}(T)}\|u\|_{F^{\frac{1}{2}+}(T)}\|u\|_{F^{s}(T)}^{2} .
$$

In the regions $G_{3}$ and $G_{6}$, we use estimates (2.10) and (2.48) to bound the corresponding terms by

$$
\|u\|_{F^{0}(T)}\|u\|_{F^{\frac{3}{4}+}(T)}\|u\|_{B^{s}(T)}^{2} .
$$

Observe that (7.15) and (7.16) are controlled by the second term on the right-hand side of (7.12).

Next, we deal with the fifth-order term corresponding to $\mathcal{M}_{k}^{2}(u)$ and observe that the one corresponding to $\mathcal{M}_{k}^{1}(u)$ could be treated similarly. It follows from estimate (2.10) that

$$
\left|\int_{\mathbb{R} \times\left[0, t_{k}\right]} \partial_{x}\left(u^{3}\right) P_{k} \partial_{x}^{-1} u P_{k} \partial_{x}^{-1} u d x\right| \lesssim\|u\|_{F^{\frac{1}{2}+}(T)}^{3}\left\|P_{k} u\right\|_{L_{T}^{\infty} L_{x}^{2}}\left\|\partial_{x}^{-1} P_{k} u\right\|_{L_{T}^{\infty} L_{x}^{2}},
$$

which leads to the bound in (17.12) after summing over $k \in \mathbb{Z}_{+} \cap[1,+\infty)$ and taking the supreme over $t_{k} \in[0, T]$. Finally, to deal with the second term on the right-hand side of (7.13), we introduce a dyadic decomposition,

$$
\partial_{x}\left(u^{3}\right)=\sum_{k_{1}, k_{2}, k_{3}} \partial_{x}\left(P_{k_{1}} u P_{k_{2}} u P_{k_{3}} u\right),
$$

and use estimates (2.10) and (2.48) to obtain the right estimate.

This finishes the proof of Proposition 7.8

\section{ACKNOWLEDGMENTS}

This research was carried out when the second author was visiting the Department of Mathematics of the University of Chicago, whose hospitality is gratefully acknowledged. The second author would like to thank Luc Molinet for helpful conversations about this work. The authors would also like to thank Tadahiro Oh for pointing out a technical mistake in a previous version of the proof of Lemma 6.3. Felipe Linares for pointing out several typos, and the anonymous referee for valuable comments and suggestions.

\section{REFERENCES}

[1] Jaime Angulo Pava, On the instability of solitary-wave solutions for fifth-order water wave models, Electron. J. Differential Equations (2003), No. 6, 18 pp. (electronic). MR.1958041 (2004k:35315)

[2] D. J. Benney, A general theory for interactions between short and long waves, Studies in Appl. Math. 56 (1976/77), no. 1, 81-94. MR0463715 (57 \#3657)

[3] J. L. Bona and R. Smith, The initial-value problem for the Korteweg-de Vries equation, Philos. Trans. Roy. Soc. London Ser. A 278 (1975), no. 1287, 555-601. MR0385355 (52 \#6219)

[4] Michael Christ, James Colliander, and Terence Tao, A priori bounds and weak solutions for the nonlinear Schrödinger equation in Sobolev spaces of negative order, J. Funct. Anal. 254 (2008), no. 2, 368-395, DOI 10.1016/j.jfa.2007.09.005. MR2376575(2008m:35327)

[5] Walter Craig, Philippe Guyenne, and Henrik Kalisch, Hamiltonian long-wave expansions for free surfaces and interfaces, Comm. Pure Appl. Math. 58 (2005), no. 12, 1587-1641, DOI 10.1002/cpa.20098. MR2177163(2006i:76012) 
[6] Walter Craig and Mark D. Groves, Hamiltonian long-wave approximations to the waterwave problem, Wave Motion 19 (1994), no. 4, 367-389, DOI 10.1016/0165-2125(94)90003-5. MR.1285131 (95h:76008)

[7] Wengu Chen, Zihua Guo, and Zeping Liu, Sharp local well-posedness for a fifth-order shallow water wave equation, J. Math. Anal. Appl. 369 (2010), no. 1, 133-143, DOI 10.1016/j.jmaa.2010.02.023. MR2643853(2011c:35396)

[8] Wengu Chen, Junfeng Li, Changxing Miao, and Jiahong Wu, Low regularity solutions of two fifth-order KdV type equations, J. Anal. Math. 107 (2009), 221-238, DOI 10.1007/s11854009-0009-0. MR2496405 (2010h:35333)

[9] Axel Grünrock, On the hierarchies of higher order $m K d V$ and $K d V$ equations, Cent. Eur. J. Math. 8 (2010), no. 3, 500-536, DOI 10.2478/s11533-010-0024-5. MR2653659 (2011d:35449)

[10] Axel Grünrock, A bilinear Airy-estimate with application to $g K d V$-3, Differential Integral Equations 18 (2005), no. 12, 1333-1339. MR2174975 (2007b:35282)

[11] Zihua Guo, Local well-posedness for dispersion generalized Benjamin-Ono equations in Sobolev spaces, J. Differential Equations 252 (2012), no. 3, 2053-2084, DOI 10.1016/j.jde.2011.10.012. MR2860610

[12] Zihua Guo, Local well-posedness and a priori bounds for the modified Benjamin-Ono equation, Adv. Differential Equations 16 (2011), no. 11-12, 1087-1137. MR.2858525(2012k:35468)

[13] Z. Guo, C. Kwak and S. Kwon, Rough solutions of the fifth-order KdV equations, J. Funct. Anal. 265 (2013), no. 11, 2791-2829. MR3096990

[14] Sebastian Herr, Well-posedness for equations of Benjamin-Ono type, Illinois J. Math. 51 (2007), no. 3, 951-976. MR2379733 (2009f:35290)

[15] A. D. Ionescu, C. E. Kenig, and D. Tataru, Global well-posedness of the KP-I initial-value problem in the energy space, Invent. Math. 173 (2008), no. 2, 265-304, DOI 10.1007/s00222008-0115-0. MR:2415308 (2009h:35368)

[16] T. Kato, Well-posedness for the fifth order KdV equation, preprint (2010), arxiv:1011.3956v1.

[17] Tosio Kato and Gustavo Ponce, On nonstationary flows of viscous and ideal fluids in $L_{s}^{p}\left(\mathbf{R}^{2}\right)$, Duke Math. J. 55 (1987), no. 3, 487-499, DOI 10.1215/S0012-7094-87-05526-8. MR.904939 (89a:35165)

[18] Carlos E. Kenig and Kenneth D. Koenig, On the local well-posedness of the BenjaminOno and modified Benjamin-Ono equations, Math. Res. Lett. 10 (2003), no. 5-6, 879-895. MR2025062 (2004j:35249)

[19] Carlos E. Kenig, Gustavo Ponce, and Luis Vega, Oscillatory integrals and regularity of dispersive equations, Indiana Univ. Math. J. 40 (1991), no. 1, 33-69, DOI 10.1512/iumj.1991.40.40003. MR.1101221(92d:35081)

[20] Carlos E. Kenig, Gustavo Ponce, and Luis Vega, On the hierarchy of the generalized KdV equations, Singular limits of dispersive waves (Lyon, 1991), NATO Adv. Sci. Inst. Ser. B Phys., vol. 320, Plenum, New York, 1994, pp. 347-356. MR1321214 (96a:35172)

[21] Carlos E. Kenig, Gustavo Ponce, and Luis Vega, Higher-order nonlinear dispersive equations, Proc. Amer. Math. Soc. 122 (1994), no. 1, 157-166, DOI 10.2307/2160855. MR 1195480 (94k:35073)

[22] Satyanad Kichenassamy, Existence of solitary waves for water-wave models, Nonlinearity 10 (1997), no. 1, 133-151, DOI 10.1088/0951-7715/10/1/009. MR1430744 (97m:35225)

[23] Herbert Koch and Daniel Tataru, A priori bounds for the $1 D$ cubic NLS in negative Sobolev spaces, Int. Math. Res. Not. IMRN 16 (2007), Art. ID rnm053, 36, DOI 10.1093/imrn/rnm053. MR2353092(2010d:35307)

[24] H. Koch and N. Tzvetkov, On the local well-posedness of the Benjamin-Ono equation in $H^{s}(\mathbb{R})$, Int. Math. Res. Not. 26 (2003), 1449-1464, DOI 10.1155/S1073792803211260. MR:1976047(2004b:35284)

[25] Soonsik Kwon, On the fifth-order KdV equation: local well-posedness and lack of uniform continuity of the solution map, J. Differential Equations 245 (2008), no. 9, 2627-2659, DOI 10.1016/j.jde.2008.03.020. MR2455780 (2010d:35309)

[26] Peter D. Lax, Integrals of nonlinear equations of evolution and solitary waves, Comm. Pure Appl. Math. 21 (1968), 467-490. MR0235310 (38 \#3620)

[27] S. P. Levandosky, A stability analysis of fifth-order water wave models, Phys. D 125 (1999), no. 3-4, 222-240, DOI 10.1016/S0167-2789(98)00245-0. MR1667522(2000b:35225)

[28] Steve Levandosky, Stability of solitary waves of a fifth-order water wave model, Phys. D 227 (2007), no. 2, 162-172, DOI 10.1016/j.physd.2007.01.006. MR2332504(2008c:35277) 
[29] Luc Molinet, Sharp ill-posedness results for the KdV and mKdV equations on the torus, Adv. Math. 230 (2012), no. 4-6, 1895-1930, DOI 10.1016/j.aim.2012.03.026. MR2927357

[30] L. Molinet, J. C. Saut, and N. Tzvetkov, Ill-posedness issues for the Benjamin-Ono and related equations, SIAM J. Math. Anal. 33 (2001), no. 4, 982-988 (electronic), DOI 10.1137/S0036141001385307. MR.1885293 (2002k:35281)

[31] Peter J. Olver, Hamiltonian and non-Hamiltonian models for water waves, Trends and applications of pure mathematics to mechanics (Palaiseau, 1983), Lecture Notes in Phys., vol. 195, Springer, Berlin, 1984, pp. 273-290, DOI 10.1007/3-540-12916-2_62. MR755731 (85k:58036)

[32] Didier Pilod, On the Cauchy problem for higher-order nonlinear dispersive equations, J. Differential Equations 245 (2008), no. 8, 2055-2077, DOI 10.1016/j.jde.2008.07.017. MR2446185 (2009f:35302)

[33] Gustavo Ponce, Lax pairs and higher order models for water waves, J. Differential Equations 102 (1993), no. 2, 360-381, DOI 10.1006/jdeq.1993.1034. MR1216734 (94c:35153)

[34] J.-C. Saut, Quelques généralisations de l'équation de Korteweg-de Vries. II (French), J. Differential Equations 33 (1979), no. 3, 320-335, DOI 10.1016/0022-0396(79)90068-8. MR543702 (82m:35134)

[35] Daniel Tataru, Local and global results for wave maps. I, Comm. Partial Differential Equations 23 (1998), no. 9-10, 1781-1793, DOI 10.1080/03605309808821400. MR1641721 (99j:58209)

Department of Mathematics, University of Chicago, Chicago, Illinois 60637

E-mail address: cek@math.uchicago.edu

Instituto de Matemática, Universidade Federal do Rio de Janeiro, Caixa Postal 68530, CEP: 21945-970, Rio DE JANEIRO, RJ, BRAZIL

E-mail address: didier@im.ufrj.br 